\title{
Verrechnungspreismethoden und Ökonomische Analyse
}

\author{
Daniel Schwerdt
}

\subsection{Einführung in die ökonomische Analyse}

Das Ziel der ökonomischen Analyse von konzerninternen Transaktionen ist die Bestimmung von angemessenen Verrechnungspreisen auf Basis der Verteilung von Funktionen und Risiken sowie eingebrachten Wirtschaftsgütern der beteiligten verbundenen Parteien. Hierbei soll sich der Steuerpflichtige am Fremdvergleichsgrundsatz orientieren. Dieser besagt, dass Preise, die zwischen verbundenen Unternehmen für Lieferungen und Leistungen verrechnet werden, den Preisen entsprechen müssen, die unabhängige Unternehmen unter sonst gleichen Marktbedingungen vereinbart haben oder hätten. Die Abgrenzung von Gewinnen aus Geschäften zwischen verbundenen Unternehmen zum Zwecke der Besteuerung richtet sich sowohl nach den deutschen Verrechnungspreisvorschriften als auch entsprechend den OECD-RL (Verrechnungspreisgrundsätze für multinationale Unternehmen und Steuerverwaltungen (1979), überarbeitete Version vom 22. Juli 2010 sowie weitere Überarbeitung verschiedener Kapitel im Rahmen des OECD BEPS Projekts veröffentlicht am 5.10.2015) nach diesem Grundsatz (siehe hierzu auch weitere Ausführungen in Kap. 2 dieses Buches).

Im Rahmen der ökonomischen Analyse wird überprüft, ob die Verrechnungspreise für konzerninterne Transaktionen mit dem Fremdvergleichsgrundsatz konform sind. Dabei ist zum einen zu prüfen, ob die Verrechnung von Leistungen dem Grunde nach gerechtfertigt ist, d. h., ob der vermeintliche Leistungsempfänger einen tatsächlichen Nutzen aus der konzernintern erbrachten Leistung bezogen hat. Diese Fragestellung stellt sich in der Praxis überwie-

D. Schwerdt $(\bowtie)$

PricewaterhouseCoopers AG Wirtschaftsprüfungsgesellschaft, Friedrich-Ebert-Anlage 35-37, 60327 Frankfurt am Main, Deutschland

E-Mail: daniel.schwerdt@de.pwc.com

(C) Der/die Herausgeber bzw. der/die Autor(en) 2016

R. Dawid (Hrsg.), Verrechnungspreise, DOI 10.1007/978-3-658-09377-8_5 
gend hinsichtlich der Verrechnung immaterieller Leistungen wie etwa Lizenzen für immaterielle Wirtschaftsgüter (Marken oder Technologie) oder Dienstleistungen. Sofern kein Nutzen für die Geschäftsaktivitäten des „Leistungsempfängers“ festzustellen ist, wäre es dem Fremdvergleichsgrundsatz folgend nicht gerechtfertigt, einen Preis für die Leistung zu bezahlen. Wenn festgestellt wird, dass ein Nutzen aus der erbrachten Leistung für das verbundene Unternehmen entsteht, ist eine Verrechnung der Leistung im Prinzip gerechtfertigt und es stellt sich die Frage nach der Höhe der Angemessenheit der Verrechnungspreise. Die Analyse der Verrechnungspreise der Höhe nach geschieht in der Regel durch einen Vergleich der untersuchten konzerninternen Transaktion mit einer vergleichbaren Transaktion zwischen unverbundenen Unternehmen. Ausgangspunkt für die ökonomische Analyse sind die Ergebnisse der Analyse der Verteilung von Funktionen und Risiken zwischen den verbundenen Transaktionspartnern (siehe hierzu gesondert Kap. 4 in diesem Buch). Auf Basis dieser Ergebnisse wird festgelegt, welche Verrechnungspreismethode für den untersuchten Fall am besten geeignet ist, um Verrechnungspreise im Einklang mit dem Fremdvergleichsgrundsatz zu bestimmen.

Dabei ist zu unterscheiden, dass die ökonomische Analyse und Anwendung der Verrechnungspreismethoden grundsätzlich für die Ex-ante Bestimmung von Verrechnungspreisen (d. h. Verrechnungspreisplanung) durchgeführt werden kann, um eine angemessene Methode sowie relevante Parameter (z. B. Preise, Margen, Lizenzsätze, Kostenbasis usw.) festzulegen, die im laufenden Geschäftsbetrieb zur Berechnung von Preisen für konzerninterne Geschäfte verwendet werden können.. Darüber hinaus kann die ökonomische Verrechnungspreisanalyse für die Ex-post Überprüfung der angewendeten Verrechnungspreise durchgeführt werden. Hier ist der Zweck der Analyse vor allem die Überprüfung der Fremdüblichkeit der gesetzten Verrechnungspreise und der Nachweis der Angemessenheit der tatsächlich angewendeten Verrechnungspreise im Rahmen der Erstellung einer Verrechnungspreisdokumentation bzw. im Laufe einer Prüfung durch die Finanzverwaltung. Dies kann grundsätzlich auch noch mit einer Verzögerung von einigen Jahren geschehen, sofern auf relevante Fremdvergleichsdaten für die untersuchten Geschäftsjahre zurückgegriffen werden kann. Auch kann auf Basis der Ex-post Überprüfung gegebenenfalls eine Anpassung der Verrechnungspreise am Ende eines Geschäftsjahres durchgeführt werden, beispielsweise wenn festgestellt wird, dass ein Unternehmen mit Routinefunktionen zu hohe bzw. zu niedrige Gewinne erzielt hat. Insofern sind die folgenden Ausführungen zur ökonomischen Analyse in diesem Kapitel so zu verstehen, dass das Methodenwerk und die entsprechende Anwendbarkeit sowohl für die Ex-ante Preisbestimmung als auch die Ex-post Überprüfung anwendbar sind. Schließlich sei hierzu jedoch angemerkt, dass sowohl die OECD als auch die Finanzverwaltung in vielen Ländern (u. a. Deutschland) mittlerweile eine eindeutige Präferenz für den sogenannten „Price-Setting Approach“ geäußert haben. ${ }^{1}$

Die nachfolgenden Abschnitte in diesem Kapitel geben einen Überblick über die in der Praxis relevanten und allgemein (zumindest in den meisten Industriestaaten und insbesondere OECD-Mitgliedsstaaten) akzeptierten Verrechnungspreismethoden. Es werden

\footnotetext{
${ }^{1}$ Vgl. Abschnitt D.1 OECD (2015), Transfer Pricing Documentation and Country-by-Country Reporting, Action 13.
} 
zunächst die Grundarten der Verrechnungspreismethoden vorgestellt, die Funktionsweise und allgemeine Anwendbarkeit der einzelnen Methoden erläutert, sowie jeweils Beispiele für die Durchführung einer ökonomischen Analyse gegeben. Desweiteren werden Besonderheiten hinsichtlich der Verrechnungspreismethoden in Deutschland dargestellt und auf Besonderheiten hinsichtlich spezifischer Transaktionstypen eingegangen.

\subsection{OECD-Verrechnungspreismethoden}

\subsubsection{Einführung in die OECD-Verrechnungspreismethoden und Überblick}

Hinsichtlich der anzuwendenden Verrechnungspreismethoden zur Einhaltung des Fremdvergleichsgrundsatzes wurde durch die OECD-Mitgliedsstaaten ein internationaler Standard entwickelt und in den OECD-RL festgehalten. Die Prämisse war hierbei, unter den teilnehmenden Mitgliedsstaaten der OECD eine gemeinsame Grundlage für die Besteuerung von Gewinnen aus internationalen, konzerninternen Transaktionen zu entwerfen, auf deren Basis Verrechnungspreise zu bestimmen und dokumentieren, sowie durch Finanzverwaltungen zu prüfen sind. Das letztendliche Ziel ist die Vermeidung von Doppelbesteuerung bzw. doppelter Nichtbesteuerung, die bei Abwesenheit eines einheitlichen Rahmenwerks und ausschließlich nationalen Regelwerken und Standards mit höherer Wahrscheinlichkeit resultieren würde. Durch die Anwendung dieser gemeinsam entwickelten Methoden soll festgestellt werden, ob die zwischen nahestehenden Unternehmen vereinbarten Preise der Höhe nach wahrscheinlich auch zwischen fremden Dritten vereinbart worden wären. Im Rahmen seiner Aufzeichnungspflichten muss der Steuerpflichtige aufzeigen, welche Verrechnungspreismethode gewählt wurde und warum er die angewandte Methode hinsichtlich der Art seiner Geschäfte und der sonstigen Verhältnisse für geeignet hält. Darüber hinaus soll anhand von für die gewählte Methode geeigneten Fremdvergleichsdaten belegt werden, dass die Verrechnungspreise fremdüblich sind.

Die OECD-RL nennen grundsätzlich zwei Arten von Verrechnungspreismethoden, die zur Einhaltung des Fremdvergleichsgrundsatzes hinsichtlich der Transaktionen zwischen verbundenen Unternehmen verwendet werden können: die geschäftsvorfallbezogenen Standardmethoden und die geschäftsvorfallbezogenen Gewinnmethoden.

Das Schaubild in Abb. 5.1 gibt einen Überblick über die Verrechnungspreismethoden gemäß den OECD-RL.

Die geschäftsvorfallbezogenen Standardmethoden stellen als Basis für den Fremdvergleich auf Preise oder Bruttomargen ab, wie sie fremde Dritte untereinander vereinbart haben. Die geschäftsvorfallbezogenen Gewinnmethoden vergleichen den Gewinn der verbundenen Unternehmen mit dem Gewinn vergleichbarer unverbundener Unternehmen, um zu bestimmen, ob die Verrechnungspreise der verbundenen Unternehmen dem Fremdvergleichsgrundsatz entsprechen. Bei der Prüfung, ob die Verrechnungspreise verbundener Unternehmen dem Fremdvergleichsgrundsatz entsprechen, kommen gemäß den deut- 


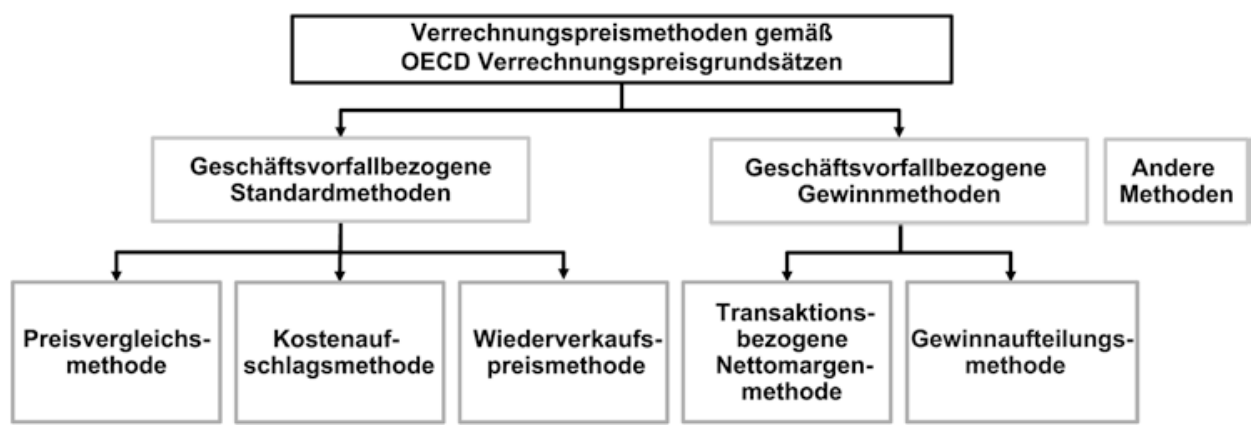

Abb. 5.1 OECD-Verrechnungspreismethoden

schen Verrechnungspreisvorschriften (siehe $\S 1$ Abs. 3 AStG) - wie auch in den OECD-RL - bevorzugt die sogenannten Standardmethoden zur Anwendung. Die Standardmethoden umfassen die folgenden Methoden:

- die Preisvergleichsmethode (engl. „Comparable Uncontrolled Price Method“)

- die Kostenaufschlagsmethode (engl. „Cost Plus Method“) und

- die Wiederverkaufspreismethode (engl. „Resale Price Method“).

Die geschäftsvorfallbezogenen Gewinnmethoden umfassen die folgenden Methoden:

- die Gewinnaufteilungsmethode (engl. „Profit Split Method“); und

- die Transaktionsbezogene Nettomargenmethode (engl. „Transactional Net Margin Method").

Neben den oben genannten Methoden sind grundsätzlich auch andere Methoden denkbar, sofern diese für den speziell untersuchten Geschäftsvorfall angemessener als die oben genannten geschäftsvorfallbezogenen Standardmethoden und die geschäftsvorfall- bezogene Gewinnmethoden sind. Als Beispiel ist hier insbesondere der hypothetische Fremdvergleich zu nennen, der auf die hypothetische Zahlungsbereitschaft des Leistungsempfängers, den hypothetischen Mindestpreis des Leistungserbringers sowie auf die Verhandlungsmacht- und alternativen der beteiligten verbundenen Parteien abstellt, um einen Verrechnungspreis zu ermitteln, der im Einklang mit dem Fremdvergleichsgrundsatz steht.

Schließlich ist anzumerken, dass das Rahmenwerk zu den oben genannten Verrechnungspreismethoden von den Mitgliedsstaaten der OECD ausgehandelt, akzeptiert und zum Teil in nationales Steuerrecht (wie z. B. in Deutschland mit § $1 \mathrm{Abs} .3 \mathrm{AStG}$ ) überführt wurde. Nichtsdestotrotz stellen die Ausführungen der OECD-RL in vielen Ländern im Rahmen von Prüfungen durch die Finanzverwaltung kein bindendes Recht dar. Vielmehr dienen die Ausführungen dem Steuerpflichtigen bzw. den Finanzverwaltungen der OECDStaaten als Richtschnur für die Bestimmung bzw. Prüfung der Verrechnungspreise, wobei viele Länder neben den OECD-RL noch weitere lokale Verrechnungspreisvorschriften haben. Jedoch haben die Ausführungen in den OECD-RL eine höhere Bedeutung im Falle von internationalen Schiedsverfahren, in denen im Rahmen von zwischenstaatlichen 
Verfahren zweier OECD Staaten die Doppelbesteuerung aufgrund von Verrechnungspreiskorrekturen in einem der beteiligten Länder beseitigt werden soll. Auch sind die Richtlinien als gemeinsamer Konsensus der Mitgliedsstaaten teilweise sehr offen formuliert, und hinterlassen somit einen gewissen Argumentations- und Interpretationsspielraum. Konkrete Handlungs- und Anwendungshinweise hinsichtlich der Methoden werden somit oft nicht gegeben und die Anwendbarkeit der Methoden hängt grundsätzlich von den relevanten Faktoren des Einzelfalls ab. Darüber hinaus ist die Akzeptanz des OECD-Verrechnungspreismethodenwerks im Prinzip auf OECD-Mitgliedsstaaten beschränkt, wobei die OECD-Methoden grundsätzlich auch von zahlreichen Nicht-Mitgliedsstaaten angewendet und akzeptiert werden. Es gibt jedoch auch Ausnahmen unter den großen Volkswirtschaften, wie z. B. Brasilien, wo von den OECD-RL abweichende Verrechnungspreismethoden (z. B. äquivalente Methoden zur Wiederverkaufspreis- bzw. Kostenaufschlagsmethode mit rechtlich vorgegebenen fixen Bruttomargen) angewendet werden müssen.

Grundsätzlich hängt die Wahl der angemessen Verrechnungspreismethode von der Verteilung der Funktionen, Risiken sowie den wesentlichen Wirtschaftsgütern (vor allem immaterielle Wirtschaftsgüter) sowie von der Verfügbarkeit zuverlässiger Fremdvergleichsdaten der an der konzerninternen Transaktion beteiligten Parteien ab. Beispielsweise könnte die transaktionsbezogene Nettomargenmethode, die auf den Vergleich von Gewinnkennziffern unabhängiger Vergleichsunternehmen abstellt, zur Ermittlung angemessener Verrechnungspreise für Routinefunktionen (siehe hierzu beispielsweise Kap. 4.4.3), wie etwa Auftragsfertigung, angewendet werden. Für Unternehmen, die hingegen als Strategieträger einzustufen sind oder über wesentliche immaterielle Wirtschaftsgüter verfügen, ist indes die transaktionsbezogene Nettomargenmethode keine geeignete Methode. Dies liegt darin begründet, dass solche Unternehmen in der Regel einzigartig sind bzw. über eine einzigartige Kombination von Funktionen, Risiken und wesentlichen (immateriellen) Wirtschaftsgütern verfügen. Der Vergleich mit Gewinnkennziffern unabhängiger Vergleichsunternehmen kann diesem Umstand jedoch nicht mit hinreichender Genauigkeit gerecht werden, sodass diese Methode als unzuverlässig für eine solche Konstellation ausscheidet. Als grundsätzliche Datenquellen für die Durchführung des Fremdvergleichs anhand der Verrechnungspreismethoden kommen Daten aus vergleichbaren Geschäften zwischen fremden Dritten (externer Vergleich) oder Daten aus vergleichbaren Geschäften zwischen dem Steuerpflichtigen und fremden Dritten (interner Vergleich) in Betracht. Die Verfügbarkeit von geeigneten Daten ist bei der Auswahl der Methode genau zu prüfen.

Die OECD-RL bezeichnen die geschäftsvorfallbezogenen Standardmethoden als die Methoden, mit denen sich am unmittelbarsten feststellen lässt, ob die konzerninternen Transaktionen fremdüblichen Bedingungen unterliegen. ${ }^{2}$ Allerdings wird anerkannt, dass die Standardmethoden in der Praxis aufgrund mangelnder empirischer Fremdvergleichswerte weniger geeignet sein können als die gewinnbezogenen Methoden. Insofern sind die OECD-RL mittlerweile von der Sichtweise abgekommen, dass die Standardmethoden bevorzugt anzuwenden sind, vielmehr wird nun die ,am besten geeignete“ Methode in den

${ }^{2}$ Vgl. Tz. 2.3 OECD-RL. 
Vordergrund gestellt. Auch gemäß den deutschen Verrechnungspreisvorschriften ist die Auswahl der angemessenen Verrechnungspreismethode insbesondere von der Verfügbarkeit geeigneter Fremdvergleichsdaten abhängig (siehe Ausführungen Kap. 5.3.1).

Für die Wahl der am besten geeigneten Methode für die Durchführung des Fremdvergleichs ist es zum einen erforderlich, die Höhe der Vergleichbarkeit von geprüften Transaktionen und Vergleichstransaktionen zu untersuchen, und zum anderen die Qualität der zugrunde liegenden Daten und Annahmen zu überprüfen. Darüber hinaus sollte auf Basis der durchgeführten Funktions- und Risikoanalyse geprüft werden, ob die Ausgestaltung der konzerninternen Transaktion an sich mit den tatsächlich ausgeübten Tätigkeiten und kontrollierten Geschäftsrisiken übereinstimmt (siehe nähere Ausführungen hierzu in Kap. 4 dieses Buches).

Zur Prüfung der Vergleichbarkeit von Transaktionen sind vor allem die folgenden Aspekte relevant: ${ }^{3}$

- Eigenschaften der übertragenen Wirtschaftsgüter oder erbrachten Dienstleistungen;

- die von den Transaktionspartnern wahrgenommenen Funktionen und Risiken sowie eingesetzte Wirtschaftsgüter;

- Vertragsbedingungen;

- die wirtschaftlichen Verhältnisse der Transaktionspartner; und

- die von den Transaktionspartnern verfolgten Geschäftsstrategien.

Die Qualität der zugrunde gelegten Daten und Annahmen ist vor dem Hintergrund der folgenden Aspekte zu prüfen:

- der Vollständigkeit und Richtigkeit der Daten;

- der Verlässlichkeit der Annahmen; und

- der Anfälligkeit des Ergebnisses in Bezug auf Mängel in den Daten und Annahmen.

Grundsätzlich ist es gemäß den OECD-RL und den deutschen Verrechnungspreisvorschriften nicht erforderlich, mehr als eine Verrechnungspreismethode anzuwenden. ${ }^{4}$

In den nachfolgenden Kapiteln werden die einzelnen OECD-Verrechnungspreismethoden näher vorgestellt sowie ihre Anwendbarkeit in der Praxis diskutiert.

\footnotetext{
${ }^{3}$ Vgl. Tz. 1.17, 1.19 bis 1.32 OECD-RL; Tz. 1.33 bis 1.40 OECD (2015), Aligning Transfer Pricing Outcomes with Value Creation, Actions 8-10; Tz. 3.4.10.2 und 3.4.12.7 VWGV; Tz. 2.1.3. VWG 1983.

${ }^{4}$ Vgl. Tz. 2.11 OECD-RL, § 2 Abs. 2 GAufzV.
} 


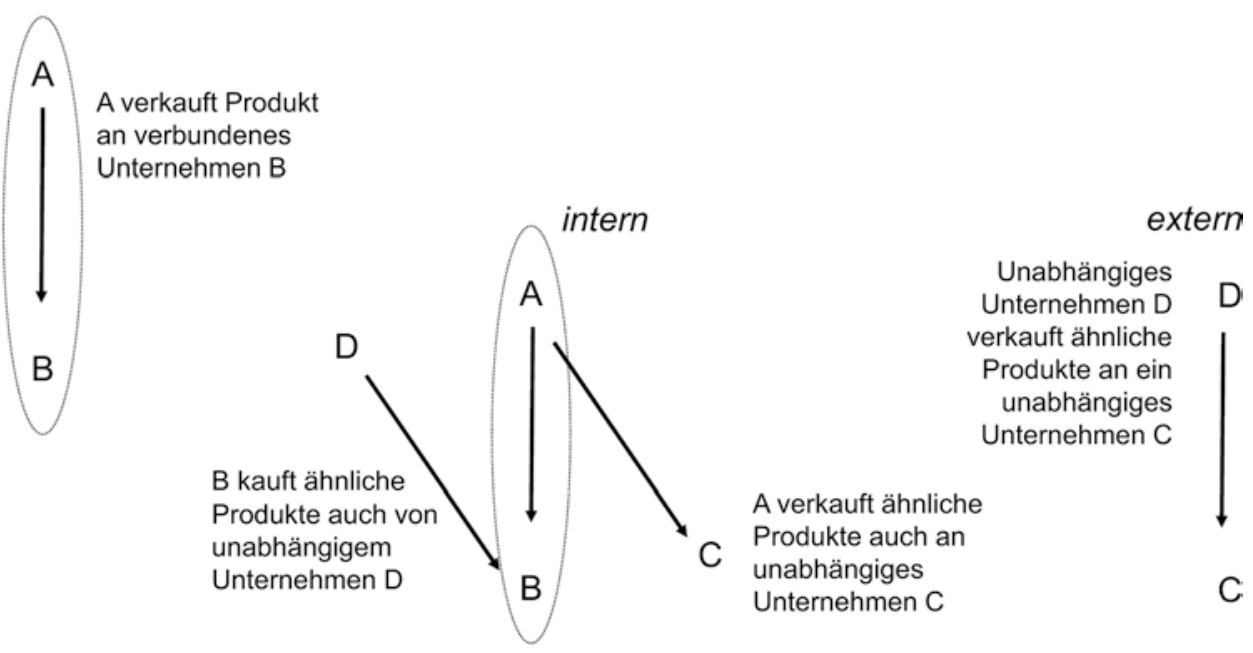

Abb. 5.2 Grundfälle der Preisvergleichsmethode

\subsubsection{Preisvergleichsmethode}

\subsubsection{Beschreibung der Preisvergleichsmethode}

Bei der Preisvergleichsmethode (oder auch „CUP-Methode“) werden zur Prüfung der Fremdüblichkeit von Transaktionen zwischen verbundenen Unternehmen direkte Preise als Vergleichskriterium herangezogen. ${ }^{5}$ Dabei werden die zwischen verbundenen Unternehmen vereinbarten Preise mit den Preisen verglichen, die von nicht verbundenen Unternehmen unter vergleichbaren Umständen vereinbart worden sind oder wären.

Beim externen Preisvergleich werden Preise, die zwischen verbundenen Unternehmen verrechnet werden, mit Preisen verglichen, wie sie am Markt zwischen fremden Dritten vereinbart worden sind. Beim internen Preisvergleich hingegen werden Preise, die zwischen verbundenen Unternehmen verrechnet werden, mit Preisen verglichen, die das verbundene Unternehmen im Rahmen vergleichbarer Transaktionen mit unverbundenen Unternehmen vereinbart hat.

Die Abb. 5.2 veranschaulicht grafisch die möglichen Grundfälle der Preisvergleichsmethode.

Die Preisvergleichsmethode ist grundsätzlich eine der verlässlichsten Verrechnungspreismethoden, wenn die Transaktionen identisch sind oder nur geringfügige, leicht quantifizierbare Unterschiede bestehen. Gemäß OECD-RL gehören zu den wichtigen Vergleichskriterien die Eigenschaften der gehandelten Güter oder Leistungen, die ausgeübten Funktionen der beteiligten Unternehmen unter Berücksichtigung von eingesetzten Vermögensgegenständen sowie übernommenen Risiken, Vertragsbedingungen sowie die wirtschaftlichen Umstände und Geschäftsstrategien der beteiligten Transaktionspartner. ${ }^{6}$

\footnotetext{
${ }^{5}$ Vgl. Tz. 2.13 OECD-RL; Tz. 2.2.2.VWG 1983.

${ }^{6}$ Vgl. Tz. 1.36 OECD (2015), Aligning Transfer Pricing Outcomes with Value Creation, Actions 8-10.
} 
Die Preisvergleichsmethode setzt ein sehr hohes Maß an Vergleichbarkeit von Produkten und Funktionen voraus. Um diesen Anforderungen zu entsprechen, sollten die Transaktionen im Hinblick auf verschiedene Charakteristika vergleichbar sein, was im Einzelfall jeweils geprüft werden muss. Die wichtigsten Kriterien sind die Folgenden:

- Vergleichbarkeit von Produkten bzw. Leistungen (z. B. Qualität, Lebenszyklus)

- Absatzvolumen (z. B. Kostendegressionseffekte, Vertriebsbündelung)

- Marktgegebenheiten (z. B. Wettbewerbssituation, politischer Rahmen)

- Vertragsbedingungen (z. B. Haftungsvereinbarungen)

- Geografische Kriterien (z. B. nationale Strukturen)

- Immaterielle Einflussfaktoren (z. B. Marken, Patente)

- Risiken (z. B. Inflationsrisiken, Währungsrisiken)

Grundsätzlich besteht die Möglichkeit, die Vergleichbarkeit von Fremdvergleichsdaten im Rahmen der Preisvergleichsmethode durch geeignete Anpassungsrechnungen zu erhöhen, durch die der Einfluss von gegebenenfalls abweichenden Faktoren auf den Preis eliminiert werden kann. ${ }^{7}$ Solche Anpassungsrechnungen sollten jedoch auf nachvollziehbaren Daten und Annahmen beruhen sowie eine entsprechende Dokumentation erstellt werden, um diese der Finanzverwaltung auf Anfrage erläutern zu können.

\subsubsection{Praktische Anwendung der Preisvergleichsmethode}

Die Preisvergleichsmethode ist den OECD-RL zufolge die direkteste und zuverlässigste Methode zur Prüfung der Fremdüblichkeit konzerninterner Transaktionen. Daher ist es in der Praxis zunächst erforderlich zu prüfen, ob diese Methode für den vorliegenden Fall anwendbar ist. Daher muss im Rahmen der ökonomischen Analyse geprüft werden, ob Preisvergleichsdaten aus vergleichbaren Geschäften zwischen fremden Dritten (externer Vergleich) oder Daten aus vergleichbaren Geschäften zwischen dem Steuerpflichtigen und fremden Dritten (interner Vergleich) verfügbar sind.

In der Praxis findet die Preisvergleichsmethode nur relativ selten Anwendung. Dies liegt insbesondere darin begründet, dass die Voraussetzungen für den Preisvergleich in vielen Fällen nicht vollständig gegeben sind. Der Preisvergleich erfordert ein sehr hohes $\mathrm{Ma} ß$ an Produktvergleichbarkeit (in der Regel identische Produkte) und Vergleichbarkeit anderer preisrelevanter Faktoren wie z. B. Transaktionsvolumen und -bedingungen. Sofern eine oder mehrere dieser Voraussetzungen nicht erfüllt sind, können sich erhebliche Unterschiede in den Preisen ergeben, sodass ein Vergleich von Preisen keine zuverlässigen Ergebnisse liefern würde. Die hohen Anforderungen an die Vergleichbarkeit von Transaktionen bedeuten jedoch in der Praxis oftmals, dass die Durchführung eines Preisvergleichs als zuverlässige Methode abgelehnt werden muss.

Die Voraussetzung für die Durchführung des externen Preisvergleichs ist die Verfügbarkeit von Preisen, die in vergleichbaren Transaktionen zwischen zwei unverbundenen

${ }^{7}$ Vgl. Tz. 2.2.2. VWG 1983. 
Unternehmen vereinbart wurden. Der externe Preisvergleich kann jedoch eher als eine theoretische Möglichkeit bezeichnet werden, da es sich in der Praxis häufig als schwierig bzw. unmöglich erweist, Preise oder entsprechende Finanzdaten für gleiche bzw. vergleichbare Transaktionen zwischen fremden Dritten zu erhalten. Aufgrund der zunehmenden Verknüpfung von konzerninternen Wertschöpfungsketten über Landesgrenzen hinweg werden in der Regel sehr spezielle Güter und Leistungen zwischen verbundenen Unternehmensteilen übertragen, etwa Halbfertigprodukte oder bestimmte Funktionen werden als Dienstleistung erbracht (z. B. Shared Service Center für die Abwicklung bestimmter Geschäftsprozesse). Für solche einzigartigen Liefer- und Leistungsbeziehungen im Rahmen der konzerninternen Wertschöpfungskette wird es in der Regel keine vergleichbaren (Markt-) Preise geben, die für einen Preisvergleich herangezogen werden können.

Ein externer Preisvergleich ist in der Regel nur bei standardisierten Massengütern (Commodities) möglich, die an Warenbörsen oder anderen öffentlichen Märkten regelmäßig gehandelt werden. Dies wurde von der OECD im Rahmen des BEPS Projekts deutlich adressiert. ${ }^{8}$ Demzufolge soll der Steuerpflichtige bei der Preissetzung für Commodity Transaktionen im Konzern möglichst auf lokale oder internationale Börsenpreisdaten zum entsprechenden Zeitpunkt der Transaktion zurückgreifen. Jedoch spielt auch hier die Vergleichbarkeit sämtlicher wirtschaftlich relevanter Merkmale der konzerninternen Transaktion mit Markttransaktionen eine Rolle, insbesondere die physischen Merkmale und Qualität der Ware, gehandeltes Volumen, Vertragsbedingungen, Zeitraum der Vereinbarung, Lieferzeitpunkt und Lieferkonditionen, Versicherungen, Transport sowie Währung. Die Preise für an Warenterminbörsen gehandelten Waren basieren in aller Regel auf standardisierten Verträgen und Bedingungen. Sofern solche Börsenpreise für die Verrechnungspreisbestimmung verwendet werden, sollte die Vergleichbarkeit der Bedingungen in der konzerninternen Transaktion mit den standardisierten Verträgen überprüft werden. Sofern es Abweichungen gibt, die sich materiell auf den Preis auswirken, sind entsprechende Anpassungen vorzunehmen um eine hinreichende Vergleichbarkeit herzustellen.

Darüber hinaus kommt ein externer Preisvergleich möglicherweise bei Darlehensbeziehungen oder anderen konzerninternen Finanzierungstransaktionen in Betracht, da Informationen über marktübliche Zinssätze bzw. andere Kapitalmarktdaten aufgrund der höheren Transparenz an den Finanzmärkten von einschlägigen Datenanbietern verfügbar sind. Dies bedeutet zwar nicht, dass für diese Daten jeweils eine vollständige Vergleichbarkeit aller relevanten Faktoren gegeben ist. Allerdings sind bei diesen Daten grundsätzlich auch Anpassungsrechnungen auf Basis von finanzmathematischen Konzepten möglich, etwa in Bezug auf internationale Zinsparitäten bzw. der Annahme effizienter Kapitalmärkte und dem Ausschluss von außergewöhnlichen Gewinnen aus Arbitragegeschäften. So sind u. a. in einem angemessenen Rahmen Anpassungsrechnungen für Zinssätze in Finanzierungstransaktionen hinsichtlich der Unterschiede in den Währungen oder Laufzeiten auf Basis anerkannter finanzmathematischer Zusammenhänge und entsprechenden

${ }^{8}$ Vgl. Tz. 2.16A bis 2.16E OECD (2015), Aligning Transfer Pricing Outcomes with Value Creation, Actions 8-10. 
Formeln möglich. Kapitalmarktdaten können somit zumindest als eingeschränkt vergleichbare Daten bezeichnet werden, die sich für die Durchführung eines Preisvergleichs in der Praxis eignen. Grundsätzlich ist es aber auch möglich, dass für bestimmte konzerninterne Finanzierungstransaktionen vollständig vergleichbare Daten ermittelt werden, etwa für kurzfristige und standardisierte Finanzprodukte.

Ferner kann unter Umständen ein externer Preisvergleich für Lizenztransaktionen anwendbar sein. Informationen über Lizenzsätze und entsprechende Verträge sind teilweise aus öffentlich zugänglichen Datenbanken (etwa RoyaltyStat, LexisNexis oder Royalty Source) erhältlich. Diese Datenbanken bedienen sich in der Regel Verträgen, die an einer US-Börse gehandelte Unternehmen im Rahmen ihrer Veröffentlichungspflichten der US Börsenaufsichtsbehörde (Security Exchange Commission) offen legen müssen. Die OECD hat im Rahmen des BEPS Projekts noch einmal klargestellt, dass dieser Ansatz zwar grundsätzlich akzeptabel ist, allerdings bei der Verwendung dieser Daten für einen externen Preisvergleich eine genaue Prüfung auf Vergleichbarkeit (insbesondere in Bezug auf das lizenzierte Gut) erforderlich ist. ${ }^{9}$ Da bei der praktischen Durchführung einer Datenbankstudie häufig nicht vollständig sichergestellt werden kann, dass die lizenzierten (immateriellen) Wirtschaftsgüter hinreichend miteinander vergleichbar sind, ist dieser datenbankbezogene Ansatz in der (Betriebsprüfungs-) Praxis zunehmend umstritten. Daher können die aus Datenbanken ermittelten Lizenzsätze auch höchstens als ,eingeschränkt vergleichbar“ bezeichnet werden.

\section{Beispiel 5.1: Externer Preisvergleich bei Darlehen}

\section{Sachverhalt:}

Die A GmbH mit Sitz in Deutschland ist ein Unternehmen in der Automobilindustrie. Die Gesellschaft verfügt aus ihren regulären Geschäftstätigkeiten über freie liquide Mittel, welche sie kurz- bzw. mittelfristig nicht benötigt, und hat ihrer Schwestergesellschaft B Srl in Italien ein Darlehen über 10 Mio. EUR mit einem fixen Zinssatz von 4\% gewährt. Das Darlehen beginnt am 1.7.2015 und hat eine feste Laufzeit von 5 Jahren. Die B Srl hat keine vergleichbaren Finanzierungstransaktionen wie etwa mit Banken.

\section{Lösung:}

Um einen fremdüblichen Preis (d. h. Zinssatz in diesem Fall) für diese Transaktion zu bestimmen, kommt ein externer Preisvergleich in Betracht, da Informationen über Finanzierungstransaktionen von Unternehmen aus öffentlichen Datenbanken grundsätzlich verfügbar sind (beispielsweise Zinssätze für die Ausgabe von Unternehmensanleihen oder Bonds). Darüber hinaus handelt es sich nicht um einen Durchreichkredit auf Seiten des deutschen Darlehensgebers, d. h., es wurde keine entsprechende Finanzierung zuvor bei einem externen Finanzdienstleister aufgenommen, die für die Verrechnungspreisfindung zu berücksichtigen wäre. Auch hat der italienische

\footnotetext{
${ }^{9}$ Vgl. Tz. 6.130 OECD (2015), Aligning Transfer Pricing Outcomes with Value Creation, Actions 8-10.
} 
Darlehensnehmer keine vergleichbaren Transaktionen mit Dritten (z. B. der Hausbank), aus denen gegebenenfalls Fremdvergleichswerte abgeleitet werden können.

Die relevanten Faktoren für die Ermittlung von Zinssätzen sind vor allem die Kreditwürdigkeit des Darlehensnehmers sowie die Darlehenskonditionen (Laufzeit, Kündigungsrechte, Besicherung, Währung usw.). Um vergleichbare Transaktionen für das vorliegende konzerninterne Darlehen aus Datenbanken zu identifizieren, ist es in einem ersten Schritt erforderlich, die Kreditwürdigkeit der B Srl zu bestimmen. Dies kann beispielsweise durch die Analyse der Finanzdaten (insbesondere Verschuldungsgrad sowie Profitabilität) der B Srl zum Zeitpunkt der Vergabe des Darlehens und Ratingmodellen von entsprechenden Anbietern (in der Regel Ratingagenturen) geschehen. Im Fall der B Srl hat die Kreditwürdigkeitsanalyse ein Rating von BB- (in Terminologie der Rating Agentur Standard \& Poors) ergeben.

In einem zweiten Schritt wird nun nach vergleichbaren Finanzierungstransaktionen auf dem Anleihenmarkt in geeigneten Datenbanken gesucht. Relevante Kriterien hierbei sind:

- Kreditwürdigkeit des Darlehensnehmers (BB-),

- Vergabezeitpunkt (1.7.2015),

- Laufzeit (5 Jahre),

- Währung (EUR),

- Kündigungsmöglichkeit (Put oder Call Option) sowie

- Industrie (Automobilindustrie).

Die Suche in der Datenbank hat in diesem Fall einen Datensatz von 10 vergleichbaren Anleihetransaktionen ergeben. Nach Berechnungen der effektiven Zinssätze dieser Vergleichstransaktionen erhält man eine Bandbreite von Zinssätzen zwischen 2,5 und 6\%. Da nicht gewährleistet ist, dass alle relevanten Faktoren der Vergleichstransaktionen vollständig vergleichbar sind, wird eine interquartile Bandbreite berechnet, um ggf. Ausreißerobservationen zu eliminieren. Die interquartile Bandbreite reicht von 3,5 bis 5\%. Somit wäre in diesem Fall anhand eines externen Preisvergleichs der Nachweis erbracht, dass der zwischen der A GmbH und B Srl vereinbarte Zinssatz in Höhe von 4\% dem Fremdüblichkeitsgrundsatz standhält.

In der Praxis kann es notwendig sein, die oben genannten Suchkriterien etwas zu erweitern, um ggf. einen größeren Datensatz an Vergleichstransaktionen zu ermitteln. Dies würde dann entsprechende Anpassungsrechnungen (beispielsweise für geringe Unterschiede in den Laufzeiten oder der Währung) erforderlich machen.

Ein interner Preisvergleich erfordert, dass zumindest eines der verbundenen Unternehmen (entweder Lieferant oder Abnehmer) neben den Konzerntransaktionen auch eng vergleichbare Transaktionen mit unverbundenen Unternehmen tätigt. Ein interner Preisvergleich ist somit nur in Fällen möglich, in denen die oben genannten Vergleichskriterien (Produktgleichheit, Transaktionsvolumen, Marktgegebenheiten, Vertragsbedingungen, geografische Kriterien, immaterielle Wirtschaftsgüter sowie übernommene Risiken) 
erfüllt sind. In der Praxis bedeutet dies, dass ein Unternehmen nahezu identische Transaktionen mit verbundenen wie unverbundenen Unternehmen haben müsste, was sehr häufig nicht der Fall ist und ein interner Preisvergleich dann als nicht anwendbar abgelehnt werden muss.

Häufig werden zwar Güter auch über unverbundene Gesellschaften abgesetzt, es ist aber oft nicht möglich ein hinreichendes Maß an Vergleichbarkeit - z. B. wegen unterschiedlicher geographischer Märkte und Transaktionsvolumina - sicherzustellen, so dass ein interner Preisvergleich für Warentransaktionen oder Dienstleistungen nicht möglich ist. Nichtdestotrotz gibt es Fälle, in denen ein interner Preisvergleich in der Praxis möglich ist, beispielsweise für konzerninterne Finanzierungstransaktionen oder auch Lizenztransaktionen.

\section{Beispiel 5.2: Interner Preisvergleich bei Lizenzen}

\section{Sachverhalt:}

Die D AG ist ein Maschinenbauunternehmen mit Sitz in Deutschland und hat Tochtergesellschaften in Frankreich, Italien, Großbritannien, Spanien, USA und Kanada. Die Forschungs- und Entwicklungstätigkeiten hinsichtlich der Maschinenprodukte werden in Deutschland durchgeführt bzw. sofern hierzu Tätigkeiten von Tochtergesellschaften übernommen werden, trägt die D AG die entsprechenden Aufwendungen. Die D AG ist somit Eigentümer sämtlicher technologischer IWG und hat entsprechende Patente angemeldet. Da es sich um große und aufwendige Anlagen handelt, werden diese im Ausland durch die Tochtergesellschaften vor Ort gefertigt und vertrieben. Die D AG hat mit allen Tochtergesellschaften Lizenzverträge hinsichtlich der technischen IWG abgeschlossen. Der Lizenzsatz beträgt 5\% vom Umsatz, der aus dem Verkauf der gefertigten Maschinen an die Kunden resultiert. Historisch bedingt hat die D AG keine Tochtergesellschaft in Australien sondern arbeitet seit vielen Jahren mit einem unabhängigen Unternehmen zusammen, welches vor Ort in Australien mit den anderen Tochtergesellschaften vergleichbare Geschäftstätigkeiten entfaltet. Das australische Unternehmen hat ebenfalls einen Lizenzvertrag mit der D AG abgeschlossen mit einem Lizenzsatz von $5 \%$ auf die Umsätze mit den in Lizenz gefertigten Maschinen.

\section{Lösung:}

Grundsätzlich käme bei dem vorliegenden Sachverhalt die Anwendung eines internen Preisvergleichs in Betracht, sofern die Verträge der D AG mit verbundenen Tochtergesellschaften sowie dem konzernfremden Unternehmen in Australien vergleichbar sind. Eine detaillierte Prüfung der Verträge hat ergeben, dass diese nahezu identische Konditionen aufweisen: es handelt sich um den gleichen Lizenzinhalt, der Vertrag ist exklusiv für ein definiertes Absatzgebiet, Kündigungsfristen und Laufzeit sind ähnlich und die Vertragsparteien haben vergleichbare Rechte und Pflichten. Darüber hinaus hat eine Analyse der Marktgegebenheiten ergeben, dass der australische Markt für diese Maschinen hinsichtlich Preisniveau, Entwicklungsgrad und Größe im Grundsatz mit den Märkten, die von den Tochtergesellschaften der D AG bedient werden, 
vergleichbar ist. Aus den vorgenannten Gründen wurde geschlossen, dass ein interner Preisvergleich in diesem Fall anwendbar ist und der Nachweis der Fremdüblichkeit des Lizenzsatzes wird somit erbracht.

Als problematisch könnte sich in dem vorgenannten Beispiel erweisen, wenn eine Tochtergesellschaft der D AG als Lizenznehmer über einen längeren Zeitraum relativ hohe Gewinne oder Verluste erwirtschaftet. Der erste Fall könnte durch eine Betriebsprüfung in Deutschland aufgegriffen werden, und es ist dann damit zu rechnen, dass die Vergleichbarkeit der Verträge durch die deutsche Finanzverwaltung sehr kritisch betrachtet und angezweifelt wird. Im zweiten Fall wäre unter Umständen mit einer intensiven Prüfung des vereinbarten Lizenzsatzes durch die entsprechende ausländische Finanzbehörde zu rechnen. Für beide Fälle sollte daher genau dokumentiert werden, welche Schritte unternommen wurden, um die tatsächliche Vergleichbarkeit der Verträge und Marktbedingungen zu prüfen und zu welchem Ergebnis die Analyse geführt hat.

\section{Beispiel 5.3: Interner Preisvergleich bei Warentransaktionen}

\section{Sachverhalt:}

Die Konsum AG ist ein Hersteller von Konsumgüterartikeln mit Sitz in Deutschland. Die Gesellschaft produziert alle Güter in Deutschland und vertreibt in Deutschland selbst an Supermarktketten, Warenhäuser, Versandhäuser und in kleinerem Umfang an Großhändler. Im Ausland vertreibt die Gesellschaft ihre Produkte über eigene Tochtergesellschaften in Frankreich, Italien, Großbritannien, Spanien und Niederlande. In kleineren geografischen Märkten, in denen sich die Gründung einer eigenen Vertriebsorganisation für den Konsum AG Konzern nicht rentieren würde, findet der Import und Vertrieb über unverbundene Distributoren statt. Dies betrifft insbesondere Finnland, Griechenland und Portugal.

\section{Lösung:}

Bei dem oben geschilderten Sachverhalt wäre grundsätzlich zu prüfen, ob ein interner Preisvergleich für die Warentransaktionen der Konsum AG möglich ist, denn die Konsum AG verkauft die gleichen Konsumgüter an verbundene Gesellschaften (Vertriebsgesellschaften im Ausland) wie an fremde Dritte (Kunden in Deutschland sowie Distributoren in Finnland, Griechenland und Portugal). Nach einer näheren Analyse der anderen wichtigen Vergleichskriterien für den Preisvergleich stellt sich jedoch heraus, dass die Produkte der Konsum AG in den verschiedenen Märkten unterschiedlich positioniert sind und nachgefragt werden. Beispielsweise können die Produkte in einigen Ländern wie Finnland, Griechenland, Großbritannien und Niederlande aufgrund der Marktgegebenheiten zu höheren Preisen an den Endkunden verkauft werden als in anderen Ländern. Darüber hinaus sind die Absatzvolumen an die Distributoren in Finnland, Griechenland und Portugal mit jährlich unter 1 Mio. EUR deutlich geringer als die Absatzvolumen an die verbundenen Vertriebsgesellschaften. Schließlich sind die Preise, welche die Konsum AG von ihren Kunden in Deutschland, d. h. vorwiegend Supermarktketten, Warenhäuser und Versandhäuser, verlangt nicht vergleichbar, da es 




Abb. 5.3 Grundfall externe Wiederverkaufspreismethode

sich nicht um die gleiche Marktstufe handelt. Während die Kunden der Konsum AG in Deutschland an Endkunden verkaufen, ist die Vertriebsgesellschaft im Ausland für den Handel an dort ansässigen Einzelhandelsunternehmen verantwortlich. Aus diesen Gründen wurde ein Preisvergleich im vorliegenden Fall als nicht anwendbar abgelehnt.

\subsubsection{Wiederverkaufspreismethode}

\subsubsection{Beschreibung der Wiederverkaufspreismethode}

Im Gegensatz zur Preisvergleichsmethode, bei der Verrechnungspreise direkt mit Fremdpreisen verglichen werden, wird bei Anwendung der Wiederverkaufspreismethode die Bruttomarge aus dem Verkauf von Produkten, die von verbundenen Unternehmen bezogen wurden, mit Bruttomargen aus vergleichbaren Transaktionen zwischen unabhängigen Unternehmen verglichen. d. h., dass der Wiederverkäufer durch den Verkauf eine angemessene Brutto-Gewinnspanne erzielen sollte.

Der Grundfall der Wiederverkaufspreismethode ist in Abb. 5.3 grafisch veranschaulicht.

Die Wiederverkaufspreismethode geht von dem Preis aus, zu dem ein von einem verbundenen Unternehmen bezogenes Produkt an fremde Dritte weiter veräußert worden ist. ${ }^{10}$ Der Preis, der für das Geschäft zwischen den verbundenen Unternehmen anzusetzen ist, wird also anhand des Marktpreises aus dem Wiederverkauf errechnet. ${ }^{11}$ Der Wiederverkaufspreis wird dann durch eine fremdübliche Bruttomarge reduziert, mit der die allgemeinen Verwaltungs- und Vertriebskosten gedeckt werden sollen und ein den Funktionen

${ }^{10}$ Vgl. Tz. 2.21 OECD-RL, Tz. 2.2.3 VWG 1983.

${ }^{11}$ Vgl. Tz. 2.2.3. VWG 1983. 
und Risiken entsprechender, angemessener Gewinn erzielt werden kann. Ein dem Fremdvergleichsgrundsatz entsprechender Verrechnungspreis ergibt sich aus dem Wiederverkaufspreis nach Abzug der angemessenen Bruttomarge und etwaigen Kosten, die sich aus dem Kauf der Leistung ergeben haben (z. B. Einfuhrzölle).

Diese Methode eignet sich insbesondere für Vertriebsgesellschaften, die Produkte weiterverkaufen ohne diese physisch zu verändern bzw. ihnen weiteren materiellen Wert hinzuzufügen. ${ }^{12}$ Schwierigkeiten bei der Anwendung der Wiederverkaufspreismethode ergeben sich dann, wenn Unternehmen neben dem Vertrieb weitere Funktionen ausüben, durch die ein wesentlicher Mehrwert generiert wird. Wenn also beispielsweise Teile des Produktionsprozesses (Zusammenbau von Komponenten etc.) von der Vertriebsgesellschaft übernommen werden, schränkt dies die Anwendbarkeit der Wiederverkaufspreismethode aufgrund der Unterschiede in den Kostenstrukturen im Vergleich zu unabhängigen Vertriebsgesellschaften erheblich ein.

Im Gegensatz zur Preisvergleichsmethode stellt die Wiederverkaufspreismethode weniger hohe Anforderungen im Hinblick auf die Produktvergleichbarkeit. Der Vergleich von Bruttomargen setzt vielmehr einen detaillierten Vergleich der Funktionen, Risiken und Vertragsbedingungen des konzerninternen und des Vergleichsgeschäfts voraus. Daher ist bei kontrollierten und nicht-kontrollierten Transaktionen desselben Wiederverkäufers (interne Wiederverkaufspreismethode) mit hoher Wahrscheinlichkeit ein höheres $\mathrm{Ma}$ an Vergleichbarkeit zu erwarten, als bei einem Vergleich zu Geschäften mit anderen Lieferanten (externe Wiederverkaufspreismethode). Die Anwendung der externen Wiederverkaufspreismethode empfiehlt sich daher nur dann, sofern nicht auf interne Vergleichstransaktionen zurückgegriffen werden kann.

Die Wiederverkaufspreismethode würde zu unzuverlässigen Ergebnissen führen, falls zwischen der kontrollierten und unkontrollierten Transaktion hinsichtlich der folgenden Punkte Unterschiede bestehen:

- der Markt- bzw. Handelsstufe (z. B. Verkauf an Vertriebsgesellschaften im Vergleich zu Einzelhändler oder Endnutzer),

- den ausgeübten Funktionen (z. B. technischer Vertrieb, Lagerhaltung, Kundenservice, Installation, Marketing), oder

- den mit der Transaktion verbundenen Risiken (z. B. Währungsrisiken, Haftungsrisiken, Transportrisiken).

Um den Mangel an Vergleichbarkeit von kontrollierten und nicht-kontrollierten Transaktionen auszugleichen, wäre es möglich hinsichtlich der folgenden Punkte Anpassungsrechnungen in angemessenem Umfang vorzunehmen:

${ }^{12}$ Vgl. Tz. 2.26 und 2.29 OECD-RL; Tz. 3.1.3. Beispiel 1 VWG 1983. 
- Lagerumschlag,

- Vertragsbedingungen,

- Frachtkosten, und

- sonstige messbare Abweichungen.

Sofern Anpassungsrechnungen vorgenommen werden, sollten diese auf nachvollziehbaren Daten und Annahmen gestützt sein. Dazu sollten diese anschließend sauber dokumentiert werden, um sie der Finanzverwaltung auf Anfrage erläutern zu können.

\subsubsection{Praktische Anwendung der Wiederverkaufspreismethode}

Die OECD-RL sehen die Wiederverkaufspreismethode vor allem als geeignete Methode zur Bestimmung von Verrechnungspreisen gegenüber Vertriebsgesellschaften an, da diese in der Regel von verbundenen Gesellschaften bezogene Waren zu Marktpreisen an externe Geschäftspartner weiter veräußern.

Grundsätzlich könnte die externe Wiederverkaufspreismethode zur Bewertung der Fremdüblichkeit der Preise zwischen verbundenen Unternehmen herangezogen werden, wenn Informationen über die in vergleichbaren Geschäften zwischen konzernfremden, unverbundenen Unternehmen generierten Bruttomargen verfügbar sind. Ein Vergleich von Bruttomargen erfordert gewöhnlich ein hohes Maß an Informationen über die Funktionen, Risiken und immaterielle Wirtschaftsgüter, die in dem Geschäft der Vertriebsaktivität eine Rolle spielen. ${ }^{13}$ Weiterhin erfordert ein externer Bruttomargenvergleich, dass die Rechnungslegung auf Ebene des zu analysierenden verbundenen Unternehmens und auf Ebene des unabhängigen Vergleichsunternehmens einheitlich erfolgt, z. B. dass die Vertriebskosten oder Materialkosten die gleichen Kostenkomponenten enthalten. Weiterhin kann der gesamte Bruttogewinn eines Unternehmens nur über dessen Jahresabschluss eingesehen werden. Bruttomargen können aber bei jedem einzelnen Produkt unterschiedlich ausfallen. Wenn diese Informationen nicht zugänglich sind, kann ein externer Bruttomargenvergleich auf Grundlage von einzelnen Produkten in der Regel nicht verlässlich durchgeführt werden.

In der Praxis erweist es sich häufig als sehr schwierig, ausreichende Informationen zur Anwendung der externen Wiederverkaufspreismethode zu erhalten. Normalerweise werden Unternehmen Daten über erzielte Bruttomargen aus einzelnen Geschäften nicht offenlegen, bzw. falls solche Daten aus irgendeinem Grund verfügbar sind, wird es dennoch schwierig sein, die Vergleichbarkeit von Funktionen und Risiken zu gewährleisten. Prinzipiell können Informationen zu aggregierten Bruttomargen aus Vertriebsaktivitäten auf Gesamtunternehmensebene aus öffentlich zugänglichen Datenbanken ermittelt werden. Da sich allerdings aus öffentlichen Quellen wie Unternehmensdatenbanken oder dem Internetauftritt des Unternehmens lediglich eingeschränkte Informationen über die Funktionen eines Unternehmens ableiten lassen, kann die für einen Bruttomargenvergleich notwendige hohe Vergleichbarkeit von Funktionen und Risiken in vielen Fällen

${ }^{13}$ Vgl. Tz. 2.21 VWG 1983 und 3.27 OECD-RL. 
nicht hinreichend sichergestellt werden. Darüber hinaus ist praktisch anzuzweifeln, ob die Daten zu Bruttomargen aus öffentlichen Datenbanken ausreichend verlässlich sind, um zu gewährleisten, dass eine einheitliche Behandlung der Kosten (Materialkosten, Vertriebs- und Verwaltungskosten) bei jedem vergleichbaren Unternehmen gegeben ist. Eine Analyse auf Ebene des Bruttogewinns erfordert allerdings implizit, dass derartige Kostenkomponenten für jedes vergleichbare Unternehmen identisch bestimmt werden. Hierzu wären jedoch sehr detaillierte Finanzdaten oder gar ein Zugang zu der Finanzbuchhaltung des Unternehmens erforderlich, um dies zu gewährleisten. Insgesamt führen die oben genannten Schwierigkeiten bei der Anwendung der externen Wiederverkaufspreismethode in der Praxis häufig dazu, dass eine verlässliche Anwendung dieser Methode in vielen Fällen nicht gegeben ist.

Als Alternative könnte die sog. modifizierte Wiederverkaufspreismethode als Bruttomargenmethode Anwendung finden. Um die oben genannten Schwierigkeiten bei der Verwendung von Bruttomargen als zuverlässige Daten für den Fremdvergleich zu umgehen, werden bei der modifizierten Wiederverkaufspreismethode die für den Fremdvergleich verwendeten Bruttomargen in einem zweistufigen Verfahren auf Basis von Nettogewinnmargen von unabhängigen Vergleichsunternehmen und der Höhe der Vertriebs- und Verwaltungskosten des analysierten verbundenen (Vertriebs-) Unternehmens in Relation zum Umsatz ermittelt. Der Fremdvergleich findet also wie bei der Wiederverkaufspreismethode auf Bruttomargenebene des untersuchten Unternehmens statt. Weitere Ausführungen zur Ermittlung von Nettogewinnmargen sind im Kapitel zur Transaktionsbezogenen Nettomargenmethode Kap. 5.2.5 zu finden.

Alternativ wäre die interne Wiederverkaufspreismethode zur Bestimmung von Verrechnungspreisen anwendbar, wenn ein Unternehmen vergleichbare Waren sowohl von verbundenen als auch unverbundenen Lieferanten unter gleichen Bedingungen einkauft und diese unverändert an fremde Dritte weitervertreibt. In diesem Fall wäre es unter Umständen möglich, einen Vergleich der erzielten Rohgewinnmargen aus beiden Transaktionen durchzuführen. Hierzu ist es erforderlich, die Vergleichbarkeit der beiden Transaktionen vor dem Hintergrund der oben aufgezählten Vergleichbarkeitskriterien genau zu prüfen. Geringe Unterschiede in der Ausgestaltung beider Transaktionen (z. B. hinsichtlich der übernommenen Funktionen und Risiken) können jedoch bereits einen erheblichen Einfluss auf die erzielten Bruttomargen haben, so dass eine Vergleichbarkeit nicht mehr gegeben sein könnte. Sofern eine solche Konstellation bei dem untersuchten Fall tatsächlich vorliegt, ergeben sich in der Praxis jedoch häufig Schwierigkeiten, da die konzernfremd bezogenen Waren häufig nur einen geringen Teil des Umsatzes ausmachen bzw. als Nebenprodukt oder Komplementärgüter zu den konzerneigenen Produkten mit angeboten werden. Dies bedeutet in der Regel, dass damit auch andere Gewinnerzielungsabsichten oder Geschäftsstrategien verfolgt werden, so dass ein Vergleich von Bruttomargen als nicht sinnvoll erscheint. Nichtdestotrotz sollte dies vor dem Hintergrund des Einzelfalls im Rahmen der Verrechnungspreisanalyse jeweils geprüft werden. 


\section{Beispiel 5.4: Wiederverkaufspreismethode bei Warentransaktionen}

\section{Sachverhalt:}

Die W AG ist ein Hersteller von Werkzeugen mit Sitz in Deutschland. Die Gesellschaft entwickelt und produziert alle Güter in Deutschland und vertreibt an Kunden in Deutschland. Aufgrund der eigenen umfangreichen Forschungs- und Entwicklungstätigkeiten verfügt die W AG über ein innovatives und qualitativ sehr hochwertiges Produktportfolio, welches am Markt eine hohe Reputation genießt. In ausländischen Märkten vertreibt das Unternehmen ihre Produkte in der Regel über konzerneigene Vertriebsgesellschaften, die sich in Frankreich, Italien, Großbritannien, Spanien und den Niederlanden befinden. Die Auslandsgesellschaften sind für die Vermarktung und Bewerbung der Produkte des Konzerns in ihrem jeweiligen Vertriebsgebiet verantwortlich. Insgesamt verkauft die D AG Werkzeuge im Wert von 500 Mio. EUR pro Jahr an ihre Vertriebstöchter im Ausland. Die Verrechnungspreise für die gelieferten Werkzeuge werden so bestimmt, dass die Vertriebstöchter eine Rohgewinnmarge von 25\% aus dem Vertrieb der Produkte an Einzelhändler in ihrem jeweiligen Markt erwirtschaften. Zur Abrundung des Produktportfolios vertreiben die ausländischen Vertriebstöchter zum Teil auch Werkzeuge und Handelsware, die sie von lokalen, unverbundenen Lieferanten beziehen.

\section{Lösung:}

Da es sich bei den konzernintern gehandelten Werkzeugen um einzigartige Produkte handelt, für die keine Fremdvergleichspreise auf der Marktstufe zwischen Produktion und Vertriebsebene verfügbar sind, scheidet ein externer und interner Preisvergleich in diesem Fall aus.

Bei dem oben geschilderten Sachverhalt wäre grundsätzlich zu prüfen, ob die interne Wiederverkaufspreismethode anwendbar ist, da die ausländischen Vertriebstöchter Waren sowohl von verbundenen (Werkzeuge der deutschen Konzernmutter) als auch unverbundenen Unternehmen (Handelsware von lokalen Zulieferern) vertreiben. Die beiden Geschäftsbeziehungen sind für jede Vertriebsgesellschaft auf Vergleichbarkeit unter den oben aufgezählten Kriterien zu prüfen. Dabei haben sich folgende Unterschiede herausgestellt:

- die Warentransaktionen mit lokalen Zulieferern haben ein deutlich geringeres Volumen und machen lediglich zwischen 5 und 10\% des Wareneinsatzes der Vertriebsgesellschaften aus

- Die Handelswaren werden nur zusätzlich zu den konzerneigenen Werkzeugen vertrieben, entsprechend sind die Margen teilweise sehr gering

- Für die Handelsware werden keine Marketing bzw. Werbemaßnahmen durchgeführt

Da sich nach einer genauen Analyse der beiden Geschäftsbeziehungen deutliche Abweichungen hinsichtlich der Volumen sowie des Funktions- und Risikoprofils ergeben haben, wird die interne Wiederverkaufspreismethode als nicht zuverlässig in diesem Fall abgelehnt. 
In einem weiteren Schritt wird geprüft, ob die Fremdüblichkeit der Rohgewinnmarge von $25 \%$, welche die Vertriebstöchter im Ausland erwirtschaften, anhand von Finanzdaten unabhängiger Vergleichsunternehmen belegt werden kann. Hierzu werden mittels Unternehmensdatenbanken 11 Unternehmen aus verschiedenen europäischen Ländern identifiziert, die ebenfalls Werkzeuge vertreiben und nicht zu einem Unternehmensverbund gehören. Es wird ermittelt, dass diese Gesellschaften Rohgewinnmargen zwischen 6 und $40 \%$ erwirtschaften. Die interquartile Bandbreite reicht von 10\% (unteres Quartil) bis 30\% (oberes Quartil) mit einem Medianwert von 17\%. Die von den Töchtern der W AG erwirtschaftete Bruttomarge von $25 \%$ liegt zwar innerhalb der ermittelten Bandbreite, jedoch erscheint zweifelhaft, ob auf Basis dieser Analyse die Fremdüblichkeit der Verrechnungspreise der W AG wirklich zuverlässig nachgewiesen ist, zumal die ermittelte Bandbreite relativ breit ist.

Die Bruttomarge der Vertriebsunternehmen hängt maßgeblich von den ausgeübten Funktionen und übernommenen Risiken ab. So muss ein Vertriebsunternehmen, welches umfangreichere Funktionen im Vertrieb übernimmt (beispielsweise eigene Marketing und Werbemaßnahmen wie Messeauftritte durchführt bzw. beratungsintensive Produkte vertreibt) eine höhere Bruttomarge erzielen, um die entsprechenden Kosten zu decken und einen Reingewinn zu erwirtschaften, als Vertriebsunternehmen mit einem vergleichsweise geringeren Funktionsprofil. Ebenso muss ein Unternehmen, das mehr Risiken im Rahmen seiner Vertriebstätigkeiten übernimmt (beispielsweise Transportrisiken, Kreditausfallrisiken, Garantierisiken) mit einer höheren Bruttomarge kalkulieren, um gegebenenfalls eintretende Risiken finanziell tragen zu können. Nach nochmaliger Überprüfung der Vergleichbarkeit der 11 identifizierten Vergleichsunternehmen anhand von Informationen aus der Datenbank sowie des Internetauftritts der Unternehmen wird festgestellt, dass detaillierte Informationen zu Funktionen und Risiken nicht für alle Unternehmen mit hinreichender Genauigkeit ermittelt werden können. Auch geben die öffentlich verfügbaren Informationen keinen Aufschluss darüber, nach welchen Rechnungslegungsvorschriften die Vergleichsunternehmen bilanziert haben bzw. ob diese einheitlich angewendet wurden. Somit ist also auch nicht gewährleistet, dass die Ermittlung des Rohgewinns aus dem Vertrieb von Waren nach einheitlichen Rechnungslegungsstandards erfolgt ist, was die Unsicherheit bzgl. der Zuverlässigkeit der ermittelten Bruttomargen weiter erhöht. Da die Informationen zu den Funktionen und Risiken der Vergleichsunternehmen insgesamt als nicht hinreichend angesehen werden, um eine hohe Vergleichbarkeit mit den Vertriebstöchtern der W AG zu gewährleisten und zusätzlich große Unsicherheit hinsichtlich der Vergleichbarkeit von Finanzdaten auf Rohgewinnebene bestehen, wird die Wiederverkaufspreismethode in diesem Fall als nicht zuverlässige Methode verworfen.

Schließlich wird in einem weiteren Schritt geprüft, ob der Fremdvergleich in diesem Fall über einen Vergleich von erzielten Nettomargen auf EBIT Basis erfolgen kann (siehe hierzu Ausführungen zur Transaktionsbezogenen Nettomargenmethode). 


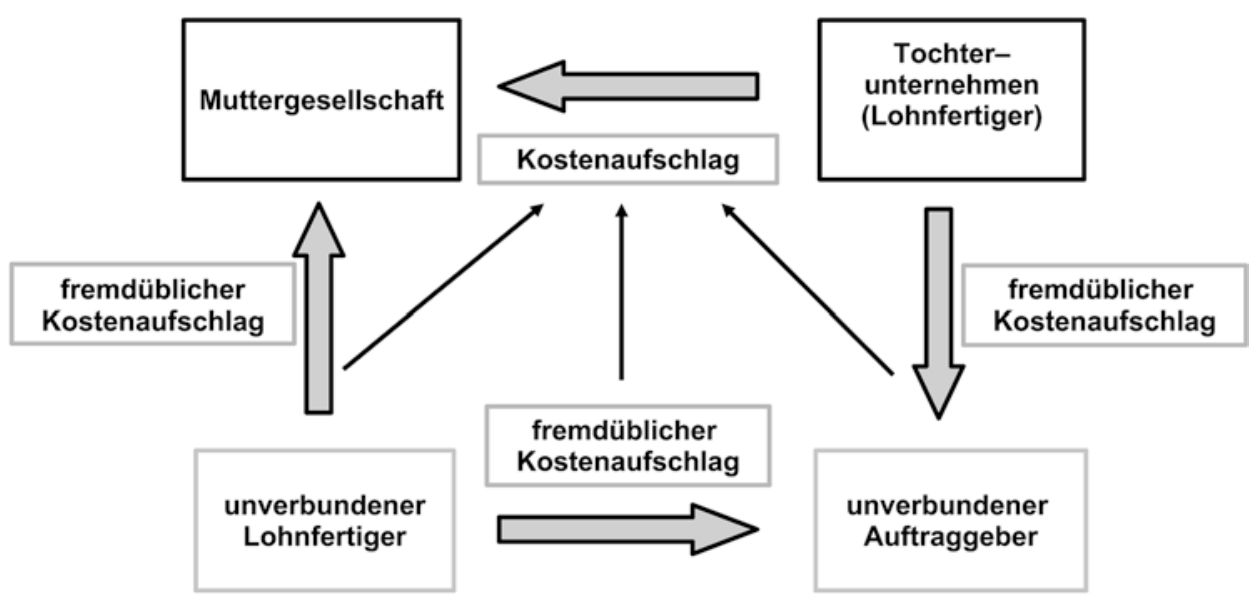

Abb. 5.4 Grundfälle der Kostenaufschlagsmethode

\subsubsection{Kostenaufschlagsmethode}

\subsubsection{Beschreibung der Kostenaufschlagsmethode}

Die Kostenaufschlagsmethode ist eine geeignete Methode, um Verrechnungspreise für die Lieferung von Waren und Leistungen von Produzenten und Dienstleistern gegenüber verbundenen Unternehmen zu bestimmen. Dabei geht sie von den Kosten aus, die einem verbundenen Unternehmen bei der Erbringung der konzerninternen Lieferungen und Leistungen entstehen. ${ }^{14}$ Zur Bestimmung des Verrechnungspreises wird dann auf die ermittelte Kostenbasis ein Gewinnaufschlag addiert, der dem Leistungserbringer einen angemessenen, den wahrgenommenen Funktionen, Risiken und Marktbedingungen entsprechenden Gewinn gewährleistet.

Der Fremdvergleich erfolgt bei dieser Methode durch den Vergleich der Kostenaufschlagssätze in einer kontrollierten und vergleichbaren unkontrollierten Transaktion statt. Die Kostenaufschlagsmethode erfordert einen detaillierten Vergleich von Produkten, Dienstleistungen, Funktionen, Risiken, Produktionskomplexität, Kostenstrukturen und immateriellen Wirtschaftsgütern der untersuchten Transaktion und der Vergleichstransaktion. Von sehr großer Bedeutung ist in diesem Zusammenhang die Vergleichbarkeit der Kostenbasis zwischen der kontrollierten und unkontrollierten Transaktion, die zur Ermittlung des fremdüblichen Aufschlagssatzes heran gezogen wird. Am wahrscheinlichsten können daher vergleichbare kontrollierte und unkontrollierte Transaktionen innerhalb desselben Herstellers oder Dienstleisters gefunden werden (d. h. interne Kostenaufschlagsmethode). Wenn solche Transaktionen nicht vorliegen, kann der Vergleich zu vergleichbaren Verkäufen oder Dienstleistungen von fremden Unternehmen gezogen werden (d. h. externe Kostenaufschlagsmethode).

Das Schaubild in Abb. 5.4 veranschaulicht grafisch die möglichen Grundfälle der Kostenaufschlagsmethode am Beispiel eines Lohnfertigers.

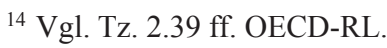


Gemäß den OECD-RL vergleicht die Kostenaufschlagsmethode den Bruttogewinn ${ }^{15}$, den verbundene Parteien in einer konzerninternen Transaktion erzielen mit dem Bruttogewinn, den fremde Dritte in einer vergleichbaren Transaktion erwirtschaften. Bei der Kostenaufschlagsmethode ist zwischen einer Analyse auf Roh- bzw. oder Bruttogewinnebene (Aufschlag auf die direkten und indirekten Kosten der Leistung) und Nettogewinnebene (Aufschlag auf sämtliche betrieblichen Aufwendungen für die Leistung) zu unterscheiden, wobei die Kostenaufschlagsmethode im Sinne der OECD-RL als Bruttogewinnanalyse gesehen wird. Die deutschen Verrechnungspreisvorschriften nehmen jedoch hinsichtlich der Ermittlung der Kostenbasis und des Gewinnzuschlags diese strenge Unterscheidung nicht vor. Gemäß der VWG 1983 sind die Kosten nach der Methode zu bestimmen, die auch zur Kalkulation von Preisen in vergleichbaren Geschäftsbeziehungen mit fremden Dritten verwendet wird. Falls keine vergleichbaren Transaktionen mit fremden Dritten bestehen, können die Kosten auch auf Basis von betriebswirtschaftlichen Grundsätzen bestimmt werden. ${ }^{16}$ Dies würde grundsätzlich auch eine Verrechnung auf Vollkostenbasis und demzufolge eine Anwendung von Nettogewinnaufschlägen beinhalten, da es betriebswirtschaftlichen Grundsätzen entspricht, auf Basis aller betrieblichen Kosten zu kalkulieren. In der Praxis kalkulieren Unternehmen sehr wohl auf Basis von betrieblichen Vollkosten (beispielsweise Kalkulation von Preisen für Teile oder Module in der Automobilzulieferer-industrie) durch Anwendung von Zuschlägen für bestimmte Gemeinkosten sowie Vertriebs- und Verwaltungskosten. Auch in der Verrechnungspreispraxis wird in Deutschland diese strenge Unterscheidung zwischen Brutto- und Nettogewinnanalyse im Prinzip nicht vorgenommen und die Kostenaufschlagsmethode häufig als Synonym für eine Brutto- als auch Nettogewinnanalyse verwendet.

Weil Unterschiede in den Funktionen und Risiken seitens der Vergleichsunternehmen den Kostenaufschlag beeinflussen können, ist ähnlich der Wiederverkaufspreismethode die Vergleichbarkeit der Funktionen und Risiken bei dieser Methode sehr wichtig. Ferner werden die Funktionen und Risiken grundsätzlich in den Finanzdaten eines Unternehmens abgebildet, weshalb verlässliche und detaillierte Finanzdaten erforderlich sind, um die darin enthaltenen Unterschiede identifizieren und ausgleichen zu können. Bei der Kostenaufschlagsmethode ist es wichtig, dass die Höhe des Kostenaufschlags auf Grundlage einer vergleichbaren Kostenbasis bestimmt wird. Schwierig kann es hierbei mitunter sein, effizienzbedingte Kostenunterschiede zu identifizieren. Daher ist eine zu Grunde liegende Annahme der Kostenaufschlagsmethode, dass die Kostenbasis für eine konzerninterne Lieferung und Leistung betriebswirtschaftlichen Grundsätzen entspricht, d. h. dass die Kostenbasis so ermittelt wurde, wie sie auch für eine Transaktion mit fremden Dritten unter marktüblichen Effizienzgesichtspunkten kalkuliert worden wäre.

Die Kostenaufschlagsmethode kann insbesondere dann zu unzuverlässigen Ergebnissen führen, falls wesentliche Unterschiede in den Geschäftsbeziehungen zwischen verbundenen und unverbunden Unternehmen bestehen. Dies kann in den folgenden Merkmalen begründet sein:

\footnotetext{
${ }^{15}$ Vgl. Tz. 2.48 und 2.53 OECD-RL.

${ }^{16}$ Vgl. Tz. 2.2.4 VWG 1983.
} 
- Eingesetzte immaterielle Wirtschaftsgüter,

- Kostenstrukturen,

- Ausgeübte Funktionen und Risiken, und

- Produkte.

Falls nur eine unzureichende Vergleichbarkeit von Transaktionen gegeben ist, können grundsätzlich in einem angemessenen Rahmen Anpassungsrechnungen vorgenommen werden, um etwa Unterschiede hinsichtlich der folgenden Aspekte auszugleichen:

- Lagerumschlag;

- Vertragsbedingungen;

- Transportkosten; und

- andere messbare Unterschiede.

\subsubsection{Praktische Anwendung der Kostenaufschlagsmethode}

Wie bereits im vorigen Absatz erläutert, ist die Kostenaufschlagsmethode ein Standardansatz zur Analyse der Fremdüblichkeit von Verrechnungspreisen für die Leistungen eines Produzenten oder Dienstleistungserbringers. Der fremdübliche Verrechnungspreis lässt sich nach dieser Methode ermitteln, indem die Produktions- oder Dienstleistungskosten mit einem angemessenen Gewinnaufschlag versehen werden. Als Vergleichsmaßstab wird hierbei der angewendete bzw. erzielte Gewinnaufschlag herangezogen und mit den Gewinnaufschlägen aus unkontrollierten vergleichbaren Transaktionen mit unverbundenen Parteien verglichen. Für die Anwendung dieser Methode wird angenommen, dass die Kostenbasis für die konzerninterne Transaktion nach betriebswirtschaftlichen Maßstäben ermittelt wurde und unter marktüblichen Bedingungen effizient ist.

In der Verrechnungspreispraxis erweist es sich häufig als schwierig, ausreichend vergleichbare Daten zur Anwendung der externen Kostenaufschlagsmethode auf Basis von Bruttogewinnaufschlägen im Sinne der OECD-RL zu ermitteln. Grundsätzlich wäre dies zwar anhand von Finanzdaten unabhängiger Unternehmen, die ein mit der untersuchten konzerninternen Transaktion vergleichbares Funktions- und Risikoprofil aufweisen, möglich. Wie allerdings bereits im Abschnitt zur Wiederverkaufspreismethode erläutert, können auf Basis frei zugänglicher Informationen in der Regel keine sehr genauen Angaben zu den übernommen Funktionen und Risiken potentieller Vergleichsunternehmen gemacht werden. Zudem stellen öffentlich zugängliche Finanzdaten von Unternehmen in der Regel nicht ausreichend verlässlich sicher, dass alle Unternehmen eine einheitliche Rechnungslegung und Kostenzuordnung (z. B. Unterscheidung Wareneinsatz, Herstellkosten und sonstigen betrieblichem Aufwand) haben. Eine Analyse auf Ebene der Bruttomargen erfordert aber implizit, dass die Kostenbasis für jedes Unternehmen einheitlich berechnet wurde. Um dies sicherzustellen, wäre es notwendig, sehr detaillierte Finanzzahlen oder Zugang zur Finanzbuchhaltung eines jeden Vergleichsunternehmens zu haben.

Aus den erläuterten Gründen ist in der Praxis eine verlässliche Anwendung der externen Kostenaufschlagsmethode mit Bruttogewinnaufschlägen aus Unternehmensdatenbanken 
häufig nicht gegeben. Alternativ wäre die Anwendbarkeit dieser Methode auf Basis von Datenbankinformationen von unabhängigen Vergleichsunternehmen zu prüfen, wenn anstatt Gewinnaufschläge auf die direkten Kosten der erbrachten Leistung (Bruttogewinnaufschlag) Gewinnaufschläge auf Vollkostenbasis, d. h. direkte und indirekte Kosten der Leistung, als Vergleichsmaßstab herangezogen werden. ${ }^{17}$ Wie oben beschrieben, erlauben auch die VWG 1983 Flexibilität hinsichtlich der Ermittlung der Kostenbasis, in dem hierzu auf betriebswirtschaftliche Grundsätze verwiesen wird. Die Verwendung von Nettogewinnaufschlägen hat den Vorteil, dass Unterschiede in den Rechnungslegungen der verschiedenen identifizierten Vergleichsunternehmen eliminiert werden, da die Klassifizierung der Kosten (als direkte oder indirekte Kosten) nicht relevant ist und der erzielte Gewinnaufschlag auf alle operativen Kosten berechnet wird. Insofern können Nettogewinnaufschläge als zuverlässiger Vergleichsmaßstab häufig aus Unternehmensdatenbanken gewonnen werden. In der Verrechnungspreispraxis in Deutschland wird daher unter der Kostenaufschlagsmethode häufig die Anwendung von Nettogewinnaufschlägen auf die Vollkosten der Leistung verstanden, sodass die Methode ähnlich zur Transaktionsbezogenen Nettomargenmethode ist (siehe hierzu die ausführlicheren Ausführungen zur Transaktionsbezogenen Nettomargenmethode in Abschn. 5.2.5).

Die Voraussetzung für die Anwendbarkeit der internen Kostenaufschlagsmethode ist, dass ein Unternehmen vergleichbare Dienstleistungs- oder Produktionsaktivitäten im Auftrag von verbundenen und unverbundenen Unternehmen erbringt. Hierzu ist es zunächst erforderlich, die Geschäftsbeziehungen auf ihre Vergleichbarkeit hinsichtlich Funktionen und Risiken, Produkten bzw. Leistungen, eingesetzter immaterieller Wirtschaftsgüter und Kostenstrukturen zu überprüfen. Da die Kostenaufschlagsmethode im Sinne der OECD-RL auf den Vergleich von Bruttogewinnen abstellt, können ähnlich der Wiederverkaufspreismethode geringe Unterschiede in der Ausgestaltung beider Transaktionen (z. B. hinsichtlich der übernommenen Funktionen und Risiken oder Ermittlung der Kostenbasis) einen erheblichen Einfluss auf die erzielten Bruttomargen haben, so dass eine Vergleichbarkeit nicht mehr gegeben sein könnte. Sofern der Vergleich der beiden Geschäftsbeziehungen ergeben hat, dass diese in hohem Maße vergleichbar sind, kann der Fremdvergleich für die Verrechnungspreise in den konzerninternen Transaktionen durch einen Vergleich der erzielten Rohgewinnmargen aus beiden Transaktionen durchgeführt werden. Ähnlich der externen Kostenaufschlagsmethode kann der Vergleich jedoch auch auf Vollkostenbasis erfolgen, so dass Nettogewinnaufschläge verglichen werden.

Neben dem Vergleich von Gewinnaufschlägen in den Geschäftsbeziehungen zwischen verbundenen und unverbundenen Parteien ist bei der Kostenaufschlagsmethode von hoher praktischer Relevanz, welche Kosten in die Kostenbasis aufzunehmen sind und ob auf alle

\footnotetext{
${ }^{17}$ Hiermit sind in der Regel alle im ordentlichen Betriebsablauf anfallenden Kosten, die im ordentlichen Betriebsergebnis enthalten sind, ohne Steuern, Zinsergebnis und außergewöhnliche Aufwendungen (d. h. EBIT) gemeint. Der individuelle Steuersatz, die Finanzierungsstruktur des Unternehmens (Verschuldungsquote) sowie ggf. einmalige Sondereffekte sollten keinen Einfluss auf die Verrechnungspreisbestimmung bei dieser Methode haben.
} 
Kosten ein Gewinnaufschlag vorzunehmen ist. Es gilt zunächst der Grundsatz, dass die Kostenbasis in der konzerninternen Transaktion mit der unkontrollierten Vergleichstransaktion vergleichbar sein muss. Im Falle der internen Kostenaufschlagsmethode wird dies in der Regel gewährleistet sein, da die Kosten anhand des gleichen Kostenrechnungssystems ermittelt werden. Im Falle eines externen Vergleichs wird man - wie oben beschrieben - in der Regel als Vergleichsmaßstab auf Vollkosten und Nettogewinnaufschläge zurückgreifen, da sonst im Prinzip keine Vergleichbarkeit der Kostenbasis gewährleistet ist. Insofern sollte der Verrechnungspreis ebenfalls auf Basis betrieblicher Vollkosten ermittelt werden. Im Falle von konzerninternen Dienstleistungen stellt sich häufig die Frage, ob der Gewinnaufschlag ebenfalls auf externe Kosten des Leistungserbringers (sogenannte ,flow-through costs“) anzuwenden ist, oder ob diese lediglich zu Kosten innerhalb des Konzerns weiterverrechnet werden sollen. Dies betrifft zumeist Kosten wie zentral getragene Kosten für IT Software, Hardware, Versicherungen, Beratungskosten, Reisekosten usw. Für diese Fälle gibt es im Prinzip keine eindeutigen Regeln in den Verrechnungspreisvorschriften. Insofern sollte im Einzelfall überprüft werden, ob ein Gewinnaufschlag ökonomisch gerechtfertigt ist und wie sich fremde Dritte verhalten würden. Beispielsweise werden zwischen unverbundenen Dritten neben einem Entgelt für erbrachte Leistungen Auslagen wie Reisekosten des Leistenden häufig separat als Spesen verrechnet und sind nicht im Dienstleistungsentgelt enthalten. Sofern der verbundene Dienstleistungserbringer in Bezug auf Leistungen und entsprechende Kosten von Dritten keine weitere Wertschöpfung leistet, wäre es aus ökonomischer Sicht nicht gerechtfertigt diese Kosten mit einem weiteren Gewinnaufschlag im Konzern zu verrechnen, da die wertschöpfende Tätigkeit in diesem Fall von einem Dritten erbracht wurde und bereits ein Gewinnelement enthält. Somit sollten nur die Kosten der eigenen Wertschöpfung (d. h. Aufwendungen, die für die Erbringung der Leistung erforderlich sind wie etwa Personalkosten und entsprechende Allgemeinkosten für Miete, IT, Verwaltung usw.) mit einem Gewinnaufschlag versehen werden.

Eine ähnliche Frage stellt sich hier in Bezug auf die Behandlung von Materialkosten für Lohn- oder Auftragsfertiger. Da ein Lohnfertiger kein Eigentum an dem verarbeitenden Material erwirbt und dieses vom Auftraggeber beigestellt wird, erhält dieser lediglich einen Gewinnaufschlag auf die eigenen Kosten der Wertschöpfung. Im Falle eines Auftragsfertigers, der Eigentum an dem zu verarbeitenden Material erwirbt, ist die Fragestellung etwas komplexer. In der Regel wird man davon ausgehen, dass die Materialkosten mit in die zugrunde liegende Kostenbasis einfließen, da der Auftragsfertiger das Eigentum und die damit verbundenen Risiken (z. B. Ausschussrisiken) übernimmt. Hat der Auftragsfertiger jedoch nur ein verbundenes Unternehmen als einzigen Abnehmer und erhält eine Vergütung auf Basis der Ist-Kosten, werden die Materialrisiken im Prinzip vom Auftraggeber getragen. In diesem Fall sollte überprüft werden, ob die Einrechnung der Materialkosten in die Kostenbasis bei der Gewinnermittlung zu einer unangemessenen Verteilung der Gewinne zwischen Auftraggeber und Auftragsfertiger führt.

Schließlich stellt sich bei Anwendung der Kostenaufschlagsmethode zudem die Frage, wie lokale Marktvorteile, etwa vergleichsweise geringe Lohn- oder Stückkosten, 
zwischen Leistungsempfänger und Leistungserbringer aufzuteilen sind. In der Praxis ist diese Fragestellung vor allem für die Vergütung von Routinefunktionen, die von verbundenen Gesellschaften in Niedriglohnländern wie z. B. Indien erbracht werden, von Relevanz. Laut OECD handelt es sich bei solchen Marktvorteilen nicht um IWG aus Verrechnungspreissicht, da diese Marktvorteile allen Marktteilnehmern im jeweiligen Land zur Verfügung stehen und nicht von Einzelnen kontrolliert werden können (siehe hierzu auch Ausführungen in Kap. 5.4.3.1). Insofern sind solche Marktvorteile nicht separat zu vergüten, vielmehr sollen angemessene Verrechnungspreise durch Verwendung von geeigneten Vergleichsdaten bestimmt werden. ${ }^{18}$ Für die Anwendung der Kostenaufschlagsmethode bedeutet dies, dass zur Bestimmung des Gewinnaufschlags in solchen Fällen auf Finanzdaten von lokalen Vergleichsunternehmen (z. B. nur indische Unternehmen) zurück zu greifen ist, da diese unabhängigen Unternehmen die jeweiligen Marktvorteile in ihren Preisen gegenüber Kunden berücksichtigt haben und sich dies entsprechend in der Profitabilität widerspiegeln sollte. In der Praxis können daher in solchen Fällen deutlich höhere Gewinnaufschläge für die Vergütung von Routinefunktionen Anwendung finden, als dies beispielsweise in voll entwickelten Industrienationen der Fall ist. Bei Verwendung eines Gewinnaufschlags, der typischerweise in voll entwickelten Industrienationen für vergleichbare Leistungen angewendet wird, würden nahezu alle Marktvorteile („Location Savings“) an den Auftraggeber weitergegeben werden, während die zusätzlichen Gewinne aus den Marktvorteilen im Rahmen von lokalen Betriebsprüfungen häufig von den jeweiligen Finanzverwaltungen in den Niedriglohnländern beansprucht werden.

\section{Beispiel 5.5: Interne Kostenaufschlagsmethode bei Auftragsfertigung}

\section{Sachverhalt:}

Die Z AG ist ein international tätiger Automobilzulieferer mit Sitz in Deutschland. Das Unternehmen entwickelt und produziert Elektronikbauteile für die Automobilindustrie. Um dem stetig steigenden Kostendruck gerecht zu werden, beschließt die Geschäftsleitung bestimmte Bauteile im osteuropäischen Ausland fertigen zu lassen und diese dann in Deutschland zu Baugruppen zu montieren. Um dies zu bewerkstelligen, übernimmt die Z AG die Elektronik S.A., ein mittelständisches Produktionsunternehmen in Rumänien, welches von nun an die von der Z AG entwickelten Bauteile als Auftragsfertiger an die Z AG liefern soll. Hierzu wird die Elektronik S.A. Rohmaterial von ihren bestehenden unverbundenen Zulieferern einkaufen und die produzierten Bauteile in der gewünschten Spezifikation und Menge an die Z AG liefern. Gleichzeitig wird die Elektronik S.A. ihre bestehenden Aufträge als Produzent weiter beliefern, um eine hohe Auslastung der Produktionskapazitäten auch im Falle einer niedrigeren Nachfrage durch die Z AG zu erreichen.

\footnotetext{
${ }^{18}$ Vgl. Tz. 1.142 OECD (2015), Aligning Transfer Pricing Outcomes with Value Creation, Actions 8-10.
} 
Die Z AG steht nun vor der Frage wie die Auftragsfertigung der Elektronik S.A. zu vergüten ist. Angedacht ist eine Vergütung auf Basis der Produktionskosten der Elektronik S.A. zuzüglich eines Gewinnaufschlags von $20 \%$.

\section{Lösung:}

Da es sich bei den im Rahmen der Auftragsfertigung gelieferten Baugruppen um einzigartige Produkte handelt, für die keine Fremdvergleichspreise auf dieser Marktstufe und unter sonst gleichen Konditionen verfügbar sind, scheidet ein externer und interner Preisvergleich in diesem Fall aus.

Unter dem gegebenen Sachverhalt wäre jedoch grundsätzlich zu prüfen, ob die interne Kostenaufschlagsmethode hier anwendbar ist, da die ausländische Produktionstochter in Rumänien sowohl Produkte für die Z AG als auch für unverbundene Kunden fertigt. Die Geschäftsbeziehungen der Elektronik S.A. mit den unverbundenen Kunden ist hierzu auf Vergleichbarkeit mit der konzerninternen, kontrollierten Transaktion unter den oben aufgezählten Kriterien zu prüfen. Die Analyse kam zu folgendem Ergebnis:

- Die Funktionen und Risiken der Elektronik S.A. sind in beiden Geschäftsarten vergleichbar. Ein geringer Unterschied ergibt sich aus der Tatsache, dass die Elektronik S.A. in kleinem Umfang Vertriebstätigkeiten in den Geschäftsbeziehungen mit unverbundenen Kunden übernimmt (Kundenansprache, Erstellung von Angeboten).

- Produkte: Zwar produziert die Elektronik S.A. unterschiedliche Elektronikbauteile für die verschiedenen Kunden. Der Produktionsprozess und die Art der Produkte sind jedoch grundsätzlich vergleichbar.

- Immaterielle Wirtschaftsgüter: Sind grundsätzlich vergleichbar, da die Elektronik S.A. jeweils als Auftragsproduzent ohne Einbringung eigener produktbezogener IWG fungiert und Produkte nach Spezifikation und Wunsch der Kunden produziert. Das eingesetzte Produktions-Know-how der Elektronik S.A. ist in beiden Geschäftsarten vergleichbar.

- Geschäftsvolumen: Ist in beiden Geschäftsarten grundsätzlich vergleichbar. Zwar erzielt die Elektronik S.A. über 50\% der Umsätze mit der Z AG, jedoch sind die gelieferten Volumen pro Produkt in beiden Geschäftsarten vergleichbar.

- Vertragsbedingungen: Zahlungsmodalitäten und Lieferkonditionen sind vergleichbar

- Markt- und Wettbewerbsbedingungen: Sind grundsätzlich vergleichbar, da die Elektronik S.A. ihre Leistungen für verbundene und unverbundene Kunden ausschließlich auf dem rumänischen Markt anbietet und erbringt.

Als Ergebnis des Transaktionsvergleichs kommt die Z AG zu dem Schluss, dass die Geschäftsbeziehungen der Elektronik S.A. mit unverbundenen Kunden grundsätzlich mit der konzerninternen Lieferungsbeziehung an die Z AG vergleichbar sind. Aus diesem Grund kann die interne Kostenaufschlagsmethode im vorliegenden Fall angewendet werden.

Die Tab. 5.1 gibt einen Überblick über die relevanten Transaktionsdaten mit fremden Dritten Kunden der Elektronik S.A. Im vorliegenden Beispiel konnten neun verschiedene, vergleichbare Produktionstransaktionen der Elektronik S.A. mit drei externen Kunden 
Tab. 5.1 Beispiel 5- Daten für Kostenaufschlagsmethode

\begin{tabular}{|c|c|c|c|c|c|c|c|c|c|}
\hline \multirow{2}{*}{$\begin{array}{l}\text { In EUR } \\
\text { Produkt- } \\
\text { typ }\end{array}$} & \multicolumn{3}{|c|}{$\begin{array}{l}\text { Externer Kunde } 1= \\
\text { Produkttyp a, b, c }\end{array}$} & \multicolumn{3}{|c|}{ Externer Kunde $2=$ d, e, f } & \multicolumn{3}{|c|}{ Externer Kunde $3=\mathrm{g}, \mathrm{h}, \mathrm{i}$} \\
\hline & $\mathrm{a}$ & $\mathrm{b}$ & $\mathrm{c}$ & $\mathrm{d}$ & e & $\mathrm{f}$ & $\mathrm{g}$ & $\mathrm{h}$ & i \\
\hline $\begin{array}{l}\text { Preis } \\
\text { pro } \\
\text { Stück }\end{array}$ & 5,40 & 3,25 & 6,05 & 4,45 & 8,34 & 5,23 & 10,12 & 9,23 & 4,45 \\
\hline $\begin{array}{l}\text { Stück- } \\
\text { kosten }\end{array}$ & 4,50 & 2,90 & 5,10 & 3,80 & 6,78 & 3,89 & 8,90 & 7,65 & 3,35 \\
\hline $\begin{array}{l}\text { Stück- } \\
\text { zahl }\end{array}$ & 90.000 & 125.000 & 78.000 & 100.000 & 56.000 & 80.000 & 35.000 & 50.000 & 100.000 \\
\hline $\begin{array}{l}\text { Produkt- } \\
\text { umsatz }\end{array}$ & 486.000 & 406.250 & 471.900 & 445.000 & 467.040 & 418.400 & 354.200 & 461.500 & 445.000 \\
\hline $\begin{array}{l}\text { Produk- } \\
\text { tions- } \\
\text { kosten }\end{array}$ & 405.000 & 362.500 & 397.800 & 380.000 & 379.680 & 311.200 & 311.500 & 382.500 & 335.000 \\
\hline $\begin{array}{l}\text { Brutto- } \\
\text { gewinn }\end{array}$ & 81.000 & 43.750 & 74.100 & 65.000 & 87.360 & 107.200 & 42.700 & 79.000 & 110.000 \\
\hline $\begin{array}{l}\text { Kosten- } \\
\text { auf- } \\
\text { schlag }\end{array}$ & $20 \%$ & $12 \%$ & $19 \%$ & $17 \%$ & $23 \%$ & $34 \%$ & $14 \%$ & $21 \%$ & $33 \%$ \\
\hline
\end{tabular}

identifiziert werden. Da die Produktionskosten für alle Produkte nach dem gleichen Schema ermittelt werden, ist eine hohe Vergleichbarkeit der Kostenstrukturen gegeben und ein Vergleich auf Bruttogewinnebene möglich.

Auf Basis des ermittelten Bruttogewinns pro Produkt und den entsprechenden Produktionskosten wird der erzielte Kostenaufschlag pro Produkt ermittelt. Die Bandbreite der Kostenaufschlagssätze reicht von 12 bis $34 \%$. Da nicht gewährleistet ist, dass alle relevanten Kriterien jeweils vollständig vergleichbar sind (eingeschränkte Vergleichbarkeit im Sinne des $\S 1$ Absatz 3 AStG, Satz 3), wird die Bandbreite mittels des Konzepts der interquartilen Bandbreite eingeengt (siehe auch VWGV Tz. 3.4.12.5). Dieses Vorgehen ist konform mit den deutschen Verrechnungsreisvorschriften und auch international (OECD-RL) üblich. Als Resultat wird eine eingeengte Bandbreite ermittelt (siehe Tab. 5.2).

Die eingeengte Bandbreite von Kostenaufschlagssätzen reicht von 17 bis $23 \%$. Somit kann davon ausgegangen werden, dass eine Bruttomarge innerhalb dieser Bandbreite mit hoher Wahrscheinlichkeit dem Fremdvergleichsgrundsatz entspricht. Entsprechend kann der Verrechnungspreis für die konzerninterne Auftragsfertigung auf Basis der Produktionskosten zuzüglich eines Gewinnaufschlags zwischen 17 und 23\% ermittelt werden. Wichtig ist in diesem Zusammenhang, dass die Kosten nach dem gleichen System bestimmt werden wie in den Transaktionen mit externen Kunden.

Gegebenenfalls könnte es notwendig sein, noch in angemessenem Umfang Anpassungsrechnungen an den Fremdvergleichsdaten vorzunehmen, z. B. für Unterschiede 
Tab. 5.2 Beispiel 5- Bandbreite Kostenaufschlagssätze

\begin{tabular}{l|l|l|l|l}
\hline Minimum (\%) & $\begin{array}{l}\text { Unteres Quartil } \\
(\%)\end{array}$ & Median (\%) & $\begin{array}{l}\text { Oberes Quartil } \\
(\%)\end{array}$ & Maximum (\%) \\
\hline 12 & 17 & 20 & 23 & 34 \\
\hline
\end{tabular}

in Volumen (Mengendegression) oder Lieferbedingungen (Preis ab Werk im Vergleich zu CIF).

Abschließend sollte noch erwähnt werden, dass der vorliegende Fall zur Veranschaulichung der Anwendungsweise der Kostenaufschlagsmethode dient. In der Praxis wird man häufig feststellen müssen, dass Konstellationen wie die oben Beschriebene sehr selten sind und diese Methode daher nicht angewendet werden kann. Sofern ein verbundenes Produktionsunternehmen sowohl verbundene als auch unverbundene Kunden beliefert, scheidet die interne Kostenaufschlagsmethode häufig aufgrund nicht vergleichbarer Volumen bzw. aufgrund Unterschiede in den eingesetzten immateriellen Wirtschaftsgütern wie Technologie, Produkteigentum, Marke, Kundenstamm usw. aus. Dies ist jeweils im Einzelfall genau zu prüfen.

\subsubsection{Transaktionsbezogene Nettomargenmethode}

\subsubsection{Beschreibung der Transaktionsbezogenen Nettomargenmethode}

Bei der Transaktionsbezogenen Nettomargenmethode (TNMM) werden die erzielten Nettogewinnmargen aus einer konzerninternen Transaktion mit den Nettogewinnmargen aus vergleichbaren Transaktionen zwischen unabhängigen Geschäftspartnern verglichen. ${ }^{19}$ Der Vergleich kann auf einzelnen Transaktionen oder auf Transaktionen, die aufgrund ihrer engen Verbundenheit zusammengefasst werden dürfen ${ }^{20}$, basieren. Dabei wird der jeweilige Nettogewinn im Verhältnis zu einer geeigneten Bezugsgröße (z. B. Kosten, Umsatz, Kapital) verglichen. Wie in den vorangegangenen Abschnitten bereits angedeutet wurde, hat die TNMM eine ähnliche Funktionsweise wie die Kostenaufschlags- oder die Wiederverkaufspreismethode, allerdings findet der Vergleich auf Nettogewinn und nicht auf Bruttogewinnebene statt. Insofern wird die TNMM in entsprechender Weise wie die Wiederverkaufspreis- oder die Kostenaufschlagsmethode angewendet. Insbesondere ist die aus der untersuchten konzerninternen Transaktion erzielte Nettogewinnspanne so zu bestimmen, wie dies der Steuerpflichtige bei vergleichbaren Geschäften mit fremden Dritten ermitteln würde (interner Vergleich). Sofern solche Geschäfte bei dem Steuerpflichtigen nicht vorliegen, kann als Vergleichsmaßstab auf die Nettogewinnspannen von unabhängigen Vergleichsunternehmen zurückgegriffen werden (externer Vergleich). In der Verrechnungspreispraxis dominiert der externe Vergleich. Voraussetzung hierfür ist die

${ }^{19}$ Vgl. Tz. 2.58 OECD-RL; zur Möglichkeit der Zusammenfassung vgl. Tz. 1.51 OECD (2015), Aligning Transfer Pricing Outcomes with Value Creation, Actions 8-10.

${ }^{20} \mathrm{Vgl}$. Tz. 3.9 bis 3.12 OECD-RL. 
Durchführung einer Funktionsanalyse des verbundenen Unternehmens und der unabhängigen Vergleichsunternehmen, um zu gewährleisten, dass die Transaktionen ausreichend vergleichbar sind und dass somit die TNMM-Analyse zu zuverlässigen Ergebnissen führt.

Wie bei allen anderen Methoden hängt die Verlässlichkeit der TNMM-Analyse vom Grad der Vergleichbarkeit der Transaktionen mit verbundenen und unverbundenen Parteien ab. Die Verlässlichkeit wird nachteilig beeinflusst, wenn Unterschiede in den Kostenstrukturen, der Geschäftserfahrung oder der Managementeffizienz bestehen. Es bedarf allerdings zur Erzielung verlässlicher Ergebnisse einer geringeren funktionalen Vergleichbarkeit als bei den geschäftsvorfallbezogenen Standardmethoden (Preisvergleichs-, Wiederverkaufspreis-, oder Kostenaufschlagsmethode). Zusätzlich ist eine geringere Produktähnlichkeit erforderlich als bei den Standardmethoden.

Es können Berichtigungen erforderlich sein hinsichtlich der Unterschiede in den folgenden Bereichen:

- Zahlungskonditionen;

- Buchhaltungsstandards:

- Lagerbestand;

- Währungsrisiko; und

- Geschäftsbedingungen.

In den meisten Fällen darf das untersuchte Unternehmen keine immateriellen oder wertvollen und einzigartigen Wirtschaftsgüter aufweisen, die es von den unabhängigen Unternehmen unterscheiden könnte. Sofern das betrachtete Unternehmen eigene wertvolle und einzigartige IWGs in die Wertschöpfungskette des Konzerns einbringt, ist die TNMM keine geeignete Methode, da eine Vergleichbarkeit mit den unabhängigen Vergleichsunternehmen nicht mehr gewährleistet werden kann.

Das Schaubild von Abb. 5.5 veranschaulicht grafisch einen möglichen Grundfall der TNMM.

In Deutschland ist die TNMM gemäß den VWGV (vgl. Tz. 3.4.10.3b) anwendbar, sofern die OECD Standardmethoden aufgrund fehlender und zuverlässiger Fremdvergleichsdaten als Methode ausscheiden. Zudem ist die TNMM auf Unternehmen mit Routinefunktionen bzw. auf Unternehmen, die in einzelnen Geschäftsbereichen Routinefunktionen durchführen und es dafür segmentierte Finanzzahlen (Spartenrechnung) gibt, anwendbar. Zudem muss ein detaillierter Nachweis der Vergleichbarkeit der ausgewählten Vergleichsunternehmen geführt werden. Für Unternehmen, die mehr als Routinefunktionen ausüben oder über wertvolle und einzigartige IWGs verfügen, ist die TNMM demzufolge nicht anwendbar.

\subsubsection{Praktische Anwendung der Transaktionsbezogenen Nettomargenmethode}

Im Vergleich zu den geschäftsvorfallbezogenen Standardmethoden erfordert die TNMM weniger detaillierte Informationen über Preise oder Bruttomargen, da sie auf Ebene von 


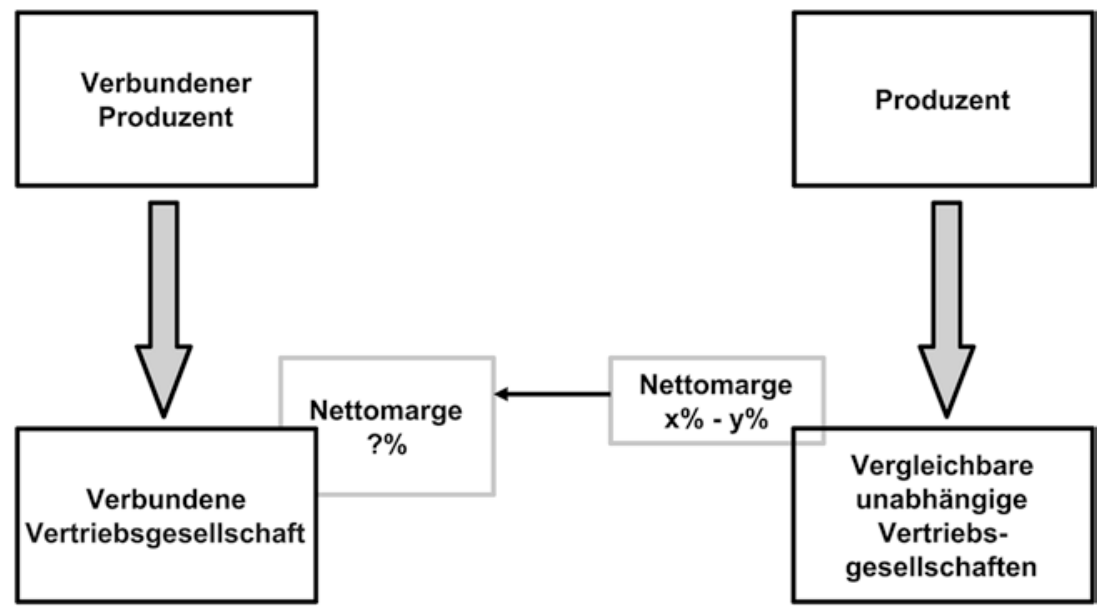

Abb. 5.5 Grundfall Transaktionsbezogene Nettomargenmethode

Nettomargen anstelle von Bruttomargen ansetzt. Wichtig ist, dass als Nettogewinn das Betriebsergebnis (also vor Abzug von Zinsaufwendungen, außerordentlichem Ergebnis und Steuern) herangezogen wird, da für Verrechnungspreiszwecke die Finanzierungsstruktur und individueller Steuersatz der betrachteten Unternehmen außer Betracht bleiben sollen. Auch sollen keine außergewöhnlichen Effekte in den Finanzdaten enthalten sein. Darüber hinaus wird die TNMM weniger nachteilig durch Unterschiede in den Rechnungslegungsmethoden des untersuchtem Unternehmens und der Vergleichsunternehmen beeinflusst. Insbesondere ist es anders als bei den Standardmethoden irrelevant, ob ein bestimmter Kostenpunkt als Wareneinsatz oder sonstige betriebliche Aufwendungen (z. B. Vertriebs, Allgemein- und Verwaltungskosten) verbucht wird. Die Kosten werden in jedem Fall von den Einkünften abgezogen, um den Reingewinn zu ermitteln. Daher erfordert die TNMM ein geringeres $\mathrm{Ma}$ an Funktions- und Produktähnlichkeit als die geschäftsvorfallbezogenen Standardmethoden, d. h., die Anforderungen an die Vergleichbarkeit sind bei der TNMM geringer als bei jeder anderen von den OECD-RL vorgeschlagenen Methode. Daher ist auch die Verfügbarkeit von Daten für den Fremdvergleich bei der TNMM deutlich besser, denn je niedriger die Anforderungen an die Vergleichbarkeit der Transaktionen sind, umso wahrscheinlicher wird es, zuverlässige Fremdvergleichsdaten zu identifizieren. Die TNMM findet in der Praxis zunehmend breitere Anwendung insbesondere für die Verrechnungspreisfindung für sogenannte Routinefunktionen, etwa Vertriebsfunktionen, Auftragsfertigung oder Dienstleistungen, da es häufig sehr schwierig ist, fremdübliche Bruttomargen oder Bruttogewinnaufschläge zu ermitteln. Die Gründe hierfür liegen vor allem darin,

- dass für den Vergleich von Bruttomargen ein relativ hohes Maß an Vergleichbarkeit der Funktionen und Risiken, Produkte und Vertragskonditionen und den damit verbundenen operativen Kosten gegeben sein muss, und 
- dass in öffentlich zugänglichen Unternehmensdatenbanken häufig keine zuverlässigen Informationen über Bruttogewinne verfügbar sind. Häufig werden nur Umsatzzahlen und Betriebsergebnis veröffentlicht.

Daher führt die Ermittlung und Analyse fremdüblicher Nettogewinne häufig zu zuverlässigeren Ergebnissen als die Anwendung der Standardmethoden. Aufgrund der relativ hohen Datenverfügbarkeit ist die TNMM somit in der deutschen und internationalen Verrechnungspreispraxis ${ }^{21}$ die am häufigsten angewendete Methode, obwohl diese streng genommen gemäß den deutschen Verrechnungspreisvorschriften einen Ausnahmefall darstellt. Demnach soll die TNMM nur für Unternehmen mit Routinefunktionen anwendbar sein, was im Prinzip auch der Sichtweise der OECD entspricht, die Routineunternehmen vor allem als Unternehmen ohne Eigentum von signifikanten einzigartigen IWG bezeichnen. Allerdings haben die deutschen Verrechnungspreisvorschriften eine engere Sichtweise in Bezug auf Routinefunktionen, und definieren den Fall von sogenannten „Mittelunternehmen“" als ein Unternehmen, das mehr als Routinefunktionen ausübt ohne „Entrepreneur“ zu sein (vgl. VWGV Tz. 3.4.10.2. c und 3.4.10.3b). In diesem Falle sei der Nachweis der Angemessenheit der Verrechnungspreise auf Basis des Vergleichs von Renditekennzahlen funktional vergleichbarer, unabhängiger Unternehmen nicht ausreichend. In einem solchen Fall soll der Fremdvergleich bei Abwesenheit von anderen Fremdvergleichsdaten anhand von Planrechnungen durchgeführt werden (vgl. VWGV Tz. 3.4.12.6), wobei diese wiederum mit den Renditekennzahlen funktional vergleichbarer, unabhängiger Unternehmen belegt werden sollen. Letztlich führt diese Thematik in der deutschen Betriebsprüfungspraxis dazu, dass es zu Diskussionen hinsichtlich der Einordnung des untersuchten Unternehmens als Routine - oder Nicht-Routine Unternehmen kommen kann. (vgl. Kap. 4.5.2.).

Da die Funktionsweise der TNMM sehr ähnlich der Kostenaufschlagsmethode bzw. der modifizierten Wiederverkaufspreismethode ist, werden diese beiden Methoden häufig als abgeleitete Methoden verwendet. Die Basis für die Verrechnungspreisfindung bilden hier ebenso Renditekennzahlen funktional vergleichbarer, unabhängiger Unternehmen. Im Falle der modifizierten Wiederverkaufspreismethode werden sodann auf Basis der ermittelten Nettomargen und den Vertriebs- und Verwaltungskosten des untersuchten Unternehmens Bruttomargen abgeleitet, auf deren Basis der Fremdvergleich durchgeführt wird. Ein solches Vorgehen entspricht im Prinzip der Meinung des BFH, der in einem Urteil

${ }^{21}$ So ist beispielsweise die TNMM (bzw. in den USA Comparable Profits Method oder CPM) die am häufigsten im Rahmen von internationalen Vorabverständigungsverfahren angewendete Verrechnungspreismethode in den USA (vgl. „Announcement and report concerning advance pricing agreements“, des Internal Revenue Services (IRS) vom 30. März 2015; http://www.irs.gov/pub/ irs-drop/a-15-11.pdf). Dies gilt sowohl für konzerninterne Transaktionen bzgl. materieller und immaterieller Güter als auch für Dienstleistungen. Für die Übertragung materieller und immaterieller Güter wurde die CPM/TNMM im Jahr 2014 in 78\% der Fälle als Verrechnungspreismethode in Vorabverständigungsverfahren verwendet. Für Dienstleistungen wurde die CPM/TNMM in $77 \%$ der Fälle verwendet. 
vom 17.2.1993 feststellte, dass eine Vertriebsgesellschaft eine Bruttomarge erwirtschaften soll, die ihr nach Abzug der Vertriebs- und Verwaltungskosten einen angemessenen Nettogewinn ermöglicht. ${ }^{22}$

Bei Anwendung der TNMM kommen je nach untersuchtem Fall verschiedene Gewinnkennzahlen für den Fremdvergleich in Betracht und das Betriebsergebnis kann zu verschiedenen anderen Größen aus der Gewinn- und Verlustrechnung bzw. Bilanz in Bezug gesetzt werden. Die Umsatzrendite wird häufig bei Vertriebsgesellschaften verwendet, der Nettogewinnaufschlag für Dienstleistungen oder Auftragsfertigung, während die Rendite auf das eingesetzte Kapital eher bei kapitalintensiven Produktionsbetrieben Verwendung findet.

Sofern die TNMM als angemessene Methode zur Analyse der Fremdüblichkeit der Verrechnungspreise einer kontrollierten Transaktion gewählt wurde, sind zur Durchführung der TNMM in der Regel die folgenden Arbeitsschritte erforderlich:

a. Auswahl des zu analysierenden Unternehmens/ Unternehmensteils

b. Zusammenfassung von Transaktionen

c. Auswahl der zu untersuchenden Wirtschaftsjahre

d. Auswahl der Vergleichsunternehmen

e. Auswahl der Gewinnkennziffer

f. Ermittlung einer Brandbreite

Die jeweiligen Arbeitsschritte werden im Folgenden kurz erläutert.

\section{a. Auswahl des zu analysierenden Unternehmens/Unternehmensteils}

Bei der Anwendung der TNMM ist in einem ersten Schritt zu ermitteln, die Finanzzahlen welches der an der kontrollierten Transaktion beteiligten Unternehmen zur Durchführung des Fremdvergleichs herangezogen werden sollen. Dies sollte der Transaktionspartner sein, für den die zuverlässigsten und belastbarsten Daten vorliegen, für den die wenigsten und verlässlichsten Anpassungen erforderlich sind und für den verlässliche Daten von unabhängigen Vergleichsunternehmen zu erhalten sind. Aus diesem Grund wird man im Regelfall die Transaktionsergebnisse derjenigen Partei untersuchen, welche das vermeintlich einfachere Funktions- und Risikoprofil aufweist. So wird man beispielsweise nicht die Ergebnisse des Transaktionspartners analysieren, welches unternehmerische Risiken trägt und über einzigartige IWG verfügt.

\section{b. Zusammenfassung von Transaktionen}

Grundsätzlich der Fremdvergleichsgrundsatz im Idealfall auf jedes einzelne Geschäft gesondert Anwendung finden. ${ }^{23}$ Wenn die einzelnen Geschäfte aber wirtschaftlich eng miteinander verbunden sind und durch eine Zusammenfassung die Aussagekraft der Analyse erhöht wird, so sollte dies als Ansatz gewählt werden. Beispielsweise kann es nach den

${ }^{22}$ BFH Urteil vom 17.2.1993, BStB1. II 1993, S. 457.

${ }^{23}$ Vgl. Tz. 3.9 OECD-RL, § 2 (3) GAufzV. 
OECD-RL sachgerechter erscheinen, Transaktionen zusammenzufassen, wenn sie miteinander verknüpft sind, viele Transaktionen in kurzer Zeit aufeinanderfolgen, z. B. bei Rechten auf Nutzung immaterieller Wirtschaftsgüter oder bei Package Deals. ${ }^{24}$ Gemäß deutschen Verrechnungspreisvorschriften ${ }^{25}$ ist eine Aggregation von Geschäftsvorfällen zulässig, wenn dies bei Geschäften zwischen fremden Dritten üblich ist, wenn die Gruppenbildung nach vorher festgelegten, nachvollziehbaren und aufgezeichneten Regeln erfolgt und die Geschäftsvorfälle gemessen an den ausgeübten Funktionen, den eingesetzten Wirtschaftsgütern und den übernommenen Risiken vergleichbar sind oder wenn es für die Prüfung der Angemessenheit im Hinblick auf die Geschäftsbeziehung weniger auf den einzelnen Geschäftsvorfall, sondern vielmehr auf die Beurteilung des Gesamtgeschäfts ankommt, z. B. bei ursächlich zusammenhängenden Geschäftsbeziehungen und bei Teilleistungen im Rahmen eines Gesamtgeschäfts.

Bei der Anwendung der TNMM wird es häufig geboten sein, eine Aggregation von einzelnen Transaktionen vorzunehmen, und das Gesamtergebnis aus der Transaktion als Vergleichsmaßstab zu verwenden. Schließlich wird auf Ebene der unabhängigen Vergleichsunternehmen ebenfalls eine aggregierte Betrachtung vorgenommen, und nicht auf Einzeltransaktionsbasis mangels vorliegender Daten. Häufig stehen die Einzeltransaktionen auch in einem engen Zusammenhang (beispielsweise dauerhafte Bereitstellung von Dienstleistungen oder Vertrieb der gesamten Produktpalette des verbundenen Produzenten), sodass eine Betrachtung von Transaktionsergebnissen für einzelne Leistungen und Waren nicht zielführend ist und nicht zu einem verlässlichen Fremdvergleich führen würde.

\section{c. Auswahl der zu untersuchenden Wirtschaftsjahre}

Da die TNMM auf die Finanzergebnisse von unabhängigen Vergleichsunternehmen als Basis für den Fremdvergleich abstellt, ist es erforderlich zu entscheiden welche, und wie viele Geschäftsjahre der Vergleichsunternehmen für den Fremdvergleich heranzuziehen sind. Häufig können die Analyseergebnisse verbessert werden, wenn Durchschnittswerte für mehrere Geschäftsjahre verwendet werden, beispielsweise wenn Faktoren wie Produktlebenszyklen, Wechselkursschwankungen oder andere Geschäftsfaktoren einen Einfluss auf die Profitabilität des zu untersuchenden Unternehmens oder der Vergleichsunternehmen haben, die nicht im Zusammenhang mit den Verrechnungspreisen stehen. Um die Auswirkung dieser verrechnungspreisfremden Faktoren zu minimieren, schlagen sowohl die OECD-RL als auch die deutschen Vorschriften einen Mehrjahresdurchschnitt vor, um einen verlässlicheren Vergleich auf Basis der TNMM durchführen zu können. ${ }^{26}$

Die Auswahl der Geschäftsjahre hängt maßgeblich von der Datenverfügbarkeit zum Zeitpunkt der Verrechnungspreisanalyse und der Zielsetzung ab. So werden in der Regel die Geschäftsergebnisse der Unternehmen in den meisten Datenbanken erst mit einer Verzögerung von einem halben bis einem Jahr erfasst. Ist das Ziel der Analyse die Ermittlung einer fremdüblichen Gewinnkennziffer für das kommende Geschäftsjahr, wird der

\footnotetext{
${ }^{24}$ Vgl. Tz. 3.9 OECD-RL.

${ }^{25}$ Vgl. § 2 (3) GAufzV, Tz. 3.4.13 VWGV.

${ }^{26}$ Vgl. Tz. 3.75 bis 3.79 OECD-RL; Tz. 3.4.12.9 VWGV.
} 
Steuerpflichtige auf historische Finanzdaten der Vergleichsunternehmen zurückgreifen müssen, wobei hier die aktuellsten verfügbaren Zahlen zu nehmen sind. Wird eine Verrechnungspreisanalyse für bereits abgelaufene Geschäftsjahre erstellt, ist es unter Umständen möglich, die gleichen Geschäftsjahre für das untersuchte Unternehmen sowie für die Vergleichsunternehmen heranzuziehen. Schließlich gibt es für die Wahl der Geschäftsjahre keine festen Regeln und im Einzelfall können die Jahre herangezogen werden, die dem Einzelfall am besten gerecht werden.

\section{d. Auswahl der Vergleichsunternehmen}

Basierend auf dem Funktions- und Risikoprofil des untersuchten Unternehmens werden zur Identifikation von unabhängigen Vergleichsunternehmen im Rahmen der TNMM häufig Unternehmensdatenbanken verwendet. Die Suche sollte auf möglichst objektiven Kriterien basieren und so aufgebaut und dokumentiert werden, dass sie im Prüfungsfall durch die Finanzverwaltung nachvollzogen werden kann. Aus diesem Grund ist es erforderlich, auf öffentlich verfügbare Datenbanken zurückzugreifen.

Als Kriterien, die sich für die Entwicklung einer Suchstrategie in einer Datenbank eignen, können die folgenden genannt werden (nicht abschließend):

- Einschränkung auf einen bestimmten geografischen Raum (siehe weitere Ausführungen unten)

- Einschränkung auf bestimmte Wirtschaftszweige

- Verwendung von eindeutigen Schlüsselwörtern als Ausschluss- oder Einschlusskriterium

- Unabhängigkeit des Unternehmens

- Verfügbarkeit von Finanzdaten

- bestimmte Finanzkennzahlen (Umsatz, relativer Lagerbestand usw.)

Die auf diese Weise aus der Datenbank identifizierten Unternehmen können jedoch noch nicht ohne weitere Prüfung als finale Vergleichsunternehmen herangezogen werden (keine reines Datenbankscreening). ${ }^{27}$ Da nicht ausgeschlossen ist, dass die Datenbankinformationen vollständig oder aktuell sind, ist es nach der Suche in der Datenbank erforderlich, die identifizierten manuell zu überprüfen, beispielsweise anhand des Internetauftritts oder weiterer Datenbanken. Schließlich sind die Auswahl der finalen Vergleichsunternehmen und die Gründe für den Ausschluss von Unternehmen noch genau zu dokumentieren, beispielsweise in tabellarischer Form.

Für die Praxis von hoher Relevanz im Zusammenhang mit Datenbankstudien ist die Frage, ob mittels einer Datenbankstudie der Fremdvergleich für eine Reihe von verbundenen Unternehmen innerhalb einer Region durchgeführt werden kann (beispielsweise für alle Vertriebsgesellschaften eines Konzerns in Europa), oder ob für jedes untersuchte Konzernunternehmen eine eigene Studie auf Basis von lokalen Vergleichsunternehmen im jeweiligen Land durchzuführen ist. Bei einem regionalen Ansatz wird die Suche nach

${ }^{27}$ Vgl. Tz. 3.4.12.3 VWGV. 
unabhängigen Vergleichsunternehmen auf die Länder innerhalb einer Region beschränkt, die grundsätzlich hinsichtlich ihres wirtschaftlichen Entwicklungsgrads und Marktumfelds vergleichbar sind (z. B. alle EU Staaten plus Norwegen und Schweiz, wobei eine Einschränkung auf bestimmte Regionen der EU wie West- bzw. Osteuropa zu empfehlen ist).

Da die Verwendung von lokalen Vergleichsunternehmen bei der Anwendung der TNMM mit deutlich höherem Aufwand und Kosten verbunden ist, hat in der Verrechnungspreispraxis der letzten zehn Jahre die Verwendung von regionalen Daten (insbesondere in der EU) dominiert. ${ }^{28}$ Auch hat sich in der Praxis gezeigt, dass bei Verwendung von lediglich landesspezifischen Vergleichsunternehmen die Anzahl der verfügbaren Vergleichsunternehmen häufig sehr gering ist und damit die Qualität der Vergleichsunternehmen (d. h. der Grad der Vergleichbarkeit der Geschäftsaktivitäten und insbesondere von Funktionen und Risiken) und die Zuverlässigkeit der Ergebnisse abnimmt. Insofern stellt sich die Frage, ob die Erhöhung der Vergleichbarkeit durch Verwendung lokaler Vergleichsunternehmen nicht wieder durch die Verwendung weniger geeigneter Vergleichsunternehmen aufgewogen wird. Allerdings gibt es einige Länder, in denen entweder gemäß Verrechnungspreisregularien oder aus der Betriebsprüfungspraxis heraus die Verwendung lokaler Vergleichsdaten stark präferiert wird. Insofern sollte die Akzeptanz von regionalen Daten in den jeweiligen Ländern im Einzelfall geklärt werden.

Die OECD hat sich im Rahmen des BEPS Projekts hinsichtlich der Verwendung von lokalen vs. Regionalen Daten klar positioniert. Da die zuverlässigsten Fremdvergleichsdaten zur Verrechnungspreisbestimmung verwendet werden sollen, sind Finanzinformationen von lokalen Vergleichsunternehmen, soweit vorhanden, gegenüber der Verwendung von regionalen Daten zu bevorzugen. ${ }^{29}$ Für die Praxis bedeutet dies nun einen erhöhten Aufwand, da jeweils im Einzelfall geprüft werden muss, ob lokale Vergleichsdaten für eine zuverlässige Analyse vorhanden sind. Wenn dies der Fall ist, sind lokale Datenbankstudien anzufertigen. Nur wenn keine zuverlässigen lokalen Daten vorhanden sind, kann demgemäß auf regionale Vergleichsdaten zurückgegriffen werden.

Basierend auf den Finanzdaten der ausgewählten finalen Vergleichsunternehmen werden Gewinnkennziffern berechnet, die für den Fremdvergleich verwendet werden. In diesem Zusammenhang wird auf das Beispiel der BSK AG zur Anwendung der TNMM von Kap. 3.4 insbesondere Abb. 3.8 verwiesen.

\section{e. Auswahl der Gewinnkennziffer}

Nach Abschluss der Suche nach unabhängigen Vergleichsunternehmen erfolgt die Analyse der Finanzdaten dieser Unternehmen. Hierzu ist es erforderlich eine Gewinnkennziffer zu wählen, die einen verlässlichen und belastbaren Vergleich zwischen den Nettogewinnen

\footnotetext{
${ }^{28} \mathrm{Vgl}$. hierzu weitere Ausführungen zur Verwendung regionaler Vergleichsunternehmen in Abschn. 3.4.3.1 dieses Buches.

${ }^{29}$ Vgl. Abschnitt D.9 OECD (2015), Transfer Pricing Documentation and Country-by-Country Reporting, Action 13.
} 
des zu untersuchenden Unternehmens und denen der Vergleichsunternehmen erlaubt. Als Beispiele seien hier die folgenden Gewinnkennziffern genannt:

$$
\text { Umsatzrendite }=\frac{\text { OperativerGewinn }(\text { EBIT })}{\text { Umsatzerlöse }} .
$$

Die Umsatzrendite misst den operativen Gewinn pro EUR an generiertem Umsatz. Als Vergleichsgröße wird häufig der operative Gewinn verwendet, der als Gewinn vor Zinsen und Steuern (,EBIT“) definiert ist. Die Umsatzrendite ist vor allem für Vertriebsgesellschaften und für Unternehmen sinnvoll, die hauptsächlich im Vertrieb ihrer Produkte tätig sind, solange der Umfang der Geschäftstätigkeit des Unternehmens in direktem Zusammenhang mit den Verkaufsumsätzen steht.

$$
\text { Berry Ratio }=\frac{\text { Bruttogewinn }}{\text { Operative Kosten }}
$$

Die Berry Ratio misst den Bruttogewinn pro EUR operative Kosten. Die Berry Ratio bietet sich vor allem für Dienstleistungsunternehmen an. Grund hierfür ist, dass viele Dienstleister, wie etwa Vertriebskommissionäre, den erreichten Absatz nicht oder nur in Teilen in ihren Finanzdaten berichten. Bei Dienstleistungsunternehmen bilden zudem die Wirtschaftsgüter das eingesetzte Kapital nur unzureichend ab. Jedoch ist diese Gewinnkennziffer sehr sensibel hinsichtlich der Unterschiede in der Einordnung von Kosten als Wareneinsatz oder sonstige betriebliche Aufwendungen.

$$
\text { Nettogewinnaufschlag }=\frac{\text { Operativer Gewinn }(\text { EBIT })}{\text { Operative Gesamtkosten }} .
$$

Der Nettogewinnaufschlag misst den operativen Gewinn pro EUR der Gesamtkosten. Der Nettogewinnaufschlag ist hauptsächlich für Dienstleistungs- oder Produktionsunternehmen geeignet, da sich ihre Geschäftstätigkeit primär in den Kosten widerspiegelt.

$$
\text { Kapitalrendite }=\frac{\text { Operativer Gewinn }(\text { EBIT })}{\text { Betriebsvermögen }} .
$$

Die Kapitalrendite misst den operativen Gewinn pro EUR des eingesetzten Betriebsvermögens, das definiert wird als das Gesamtvermögen abzüglich Investitionen, wie solche in Tochterunternehmen und Wertpapiere. Die Kapitalrendite ist vor allem für Unternehmen geeignet, bei denen das Betriebsvermögen wie Grundstücke, Fabriken und Anlagen einen starken Einfluss auf die Profitabilität hat.

Die Auswahl der Renditekennziffer ist jeweils einzelfallbezogen zu treffen. Dabei ist die Kennziffer zu wählen, die die Zuverlässigkeit des Fremdvergleichs noch weiter erhöht. 


\section{f. Ermittlung einer Bandbreite}

Als Ergebnis der Datenbankstudie resultieren je nach gesuchten Unternehmenstypus typischerweise zwischen 10 und 30 Vergleichsunternehmen. Nach Berechnung einer geeigneten Renditekennziffer auf Basis der Finanzzahlen der identifizierten Unternehmen für die ausgewählten Jahre (siehe Schritt c) oben) erhält man eine Bandbreite von Fremdvergleichswerten. Der Vergleich der Ergebnisse der Vergleichsunternehmen mit den Ergebnissen des zu untersuchenden Unternehmens ist eine Grundvoraussetzung für die Überprüfung des Fremdvergleichsgrundsatzes. Daher ist es entscheidend, dass die Vergleichsunternehmen und das untersuchte abhängige Unternehmen ihre Geschäftsaktivitäten unter denselben oder zumindest ähnlichen Bedingungen ausüben. Alle Faktoren, die die Verrechnungspreise und demnach auch die Gewinnkennziffernbeeinflussen könnten, müssen beachtet und gegebenenfalls durch Anpassungen berücksichtigt werden. In der Praxis wird man als Resultat der Anwendung einer Verrechnungspreismethode häufig nicht einen einzelnen Preis oder eine einzelne Marge erhalten, sondern eine Reihe von Daten, die jeweils gleich zuverlässige Werte darstellen. Dies liegt zum einen daran, dass bei der Vergleichbarkeitsanalyse nicht jeder Beurteilungsmaßstab hundertprozentig überprüft und Vergleichbarkeit hergestellt werden kann. Zum anderen ist auch nicht gewährleistet, dass sich fremde Dritte in Preisverhandlungen immer auf den gleichen Preis einigen würden. Insofern erkennen die OECD-RL an, dass häufig die Berechnung einer Bandbreite von Preisen anstatt der Angabe eines einzelnen Wertes für die Verrechnungspreisbestimmung erforderlich ist.

Gemäß deutscher Verrechnungspreisvorschriften ${ }^{30}$ kann eine solche Bandbreite nur dann in vollem Umfang berücksichtigt werden, wenn aufgrund zuverlässiger und vollständiger Informationen feststeht, dass eine uneingeschränkte Vergleichbarkeit der Geschäftsbedingungen besteht. Besteht die Bandbreite nur aus eingeschränkt vergleichbaren Werten oder steht die uneingeschränkte Vergleichbarkeit wegen Informations- und Datenmängeln nicht hinreichend zuverlässig fest, ist keine ausreichende Vergleichbarkeit gegeben. Aus diesem Grund wird in der Regel für den Fremdvergleich nur die eingeengte Bandbreite der fremdüblichen Werte verwendet. Dabei wird häufig die interquartile Bandbreite angewendet, die aus den mittleren 50\% der Ergebnisse der Vergleichsunternehmen besteht. Die Einschränkung der Bandbreite mithilfe anderer geeigneter statistischer Methoden ist jedoch ebenfalls möglich.

\section{Ergänzung des Beispiels 5.4: TNMM bei Warentransaktionen}

\section{Sachverhalt:}

Siehe detaillierte Beschreibung des Sachverhalts in Kap. 5.2.3 zur Wiederverkaufspreismethode. Die in Deutschland ansässige W AG entwickelt und produziert Werkzeuge. Diese werden in ausländischen Märkten über eigene Vertriebsgesellschaften in Frankreich, Italien, Großbritannien, Spanien und Niederlande vertrieben. Die interne

${ }^{30}$ Vgl. Tz. 3.4.12.5 VWGV und $\S 1$ (3) AStG. 
und externe Wiederverkaufspreismethode wurde hier aufgrund mangelnder zuverlässiger Daten als nicht anwendbare Methode verworfen.

\section{Lösung:}

Nachdem die Anwendung der geschäftsvorfallbezogenen Standardmethoden in diesem Fall abgelehnt wurde, wird in einem weiteren Schritt geprüft, ob der Fremdvergleich über einen Vergleich von erzielten Nettomargen auf EBIT Basis erfolgen kann. Wie oben erläutert, erfordert die TNMM ein geringeres Maß an Vergleichbarkeit von Funktionen- und Risiken sowie Produkten als die geschäftsvorfallbezogenen Standardmethoden, d. h., die Anforderungen an die Vergleichbarkeit sind bei der TNMM geringer als bei jeder anderen von den OECD-RL vorgeschlagenen Methoden. Darüber hinaus wird die TNMM weniger nachteilig durch Unterschiede in den Rechnungslegungsmethoden von untersuchtem Unternehmen und den Vergleichsunternehmen beeinflusst. Aus diesen Gründen wird die TNMM im vorliegenden Fall als eine anwendbare Methode betrachtet.

Für die neun identifizierten Vergleichsunternehmen werden nun, anstatt Bruttomargen, Nettomargen im 3-Jahresdurchschnitt der letzten verfügbaren Finanzjahre berechnet. Die Ergebnisse sind in Tab. 5.3 dargestellt.

Die Bandbreite der erzielten Nettomargen der Vergleichsunternehmen reicht von $-0,8$ bis $14,2 \%$. Da nicht gewährleistet ist, dass es sich um vollständig vergleichbare Daten handelt, wird die Bandbreite mittels des Konzepts der interquartilen Bandbreite eingeengt, um die Analyse zu verfeinern. Das Resultat der Einengung der Bandbreite ist in Tab. 5.4 dargestellt.

Die eingeschränkte Bandbreite von Nettomargen reicht von 2,0 bis 5,1\%. Somit kann davon ausgegangen werden, dass eine Nettomarge aus den analysierten Vertriebstätigkeiten innerhalb dieser Bandbreite mit hoher Wahrscheinlichkeit dem Fremdvergleichsgrundsatz entspricht. Entsprechend können nun die erzielten Nettomargen der Vertriebsgesellschaften in Frankreich, Italien, Großbritannien, Spanien und Niederlande mit der ermittelten Bandbreite verglichen werden. Sofern diese innerhalb dieser Bandbreite liegen, ist der Fremdvergleich erbracht. Bei einer Abweichung sollten die

Tab. 5.3 Erweiterung Beispiel 5.4 Nettomargen der Vergleichsunternehmen

\begin{tabular}{l|l|l|l|l|l|l|l|l|l}
\hline InTEUR & AGmbH & B Srl & C Ltd. & D AG & E Spa & F S.A. & G Ltd. & H N.V. & I B.V. \\
\hline Umsatz & 50.000 & 30.000 & 35.000 & 98.000 & 15.000 & 24.000 & 78.000 & 65.000 & 33.000 \\
\hline EBIT & 1000 & 800 & 700 & 3000 & 1000 & 3400 & 4000 & 500 & 300 \\
\hline $\begin{array}{l}\text { Umsatz- } \\
\text { rendite }\end{array}$ & $2,0 \%$ & $2,7 \%$ & $2,0 \%$ & $3,1 \%$ & $6,7 \%$ & $14,2 \%$ & $5,1 \%$ & $-0,8 \%$ & $0,9 \%$ \\
\hline
\end{tabular}

Tab. 5.4 Tabelle 3: Erweiterung Beispiel 5.4 Bandbreite Nettomargen der Vergleichsunternehmen

\begin{tabular}{l|l|l|l|l}
\hline Minimum (\%) & Unteres Quartil (\%) & Median (\%) & Oberes Quartil (\%) & Maximum (\%) \\
\hline$-0,8$ & 2,0 & 2,7 & 5,1 & 14,2 \\
\hline
\end{tabular}


entsprechenden Ursachen analysiert und dokumentiert werden. Unter Umständen ist es auch sinnvoll einen Mehrjahresvergleich mit den Finanzdaten der untersuchten Unternehmen durchzuführen.

\subsubsection{Gewinnaufteilungsmethode}

\subsubsection{Beschreibung der Gewinnaufteilungsmethode}

Geschäftsbeziehungen zwischen zwei verbundenen Parteien (Gesellschaften oder Stammhaus und Betriebsstätte) können so eng miteinander verbunden und voneinander abhängig sein, dass eine separate Bewertung eines einzelnen Transaktionszweiges nicht durchführbar ist. ${ }^{31}$ Das kann insbesondere dann der Fall sein, wenn der Gewinn aus den Transaktionen auf wertvolle und einzigartige immaterielle Wirtschaftsgüter zurückzuführen ist, die beide Transaktionspartner gemeinsam erschaffen haben und verwenden. In diesen Fällen - in denen auch unabhängige Dritte ein Joint Venture und eine Gewinnaufteilung vereinbaren würden - wäre die Anwendung der Gewinnaufteilungsmethode angemessen.

Zur Anwendung der Gewinnaufteilungsmethode muss zunächst das zwischen den verbundenen Unternehmen aufteilbare, gemeinsam erwirtschaftete Transaktionsergebnis ermittelt werden. Im nächsten Schritt müssen, abhängig von der gewählten Variante der Gewinnaufteilungsmethode, entweder eine vergleichbare Gewinnaufteilung, die zwischen unverbundenen Unternehmen angewendet wird, oder aber objektive Wertschöpfungskriterien als Verteilungsmaßstab bestimmt werden. Ausgehend von diesen Werten werden die Transaktionsergebnisse entsprechend des Verteilungsmaßstabes der Drittunternehmen bzw. der jeweiligen Wertschöpfungsbeiträge verteilt.

Wie die OECD-RL klarstellen ${ }^{32}$, besteht ein Vorteil der Gewinnaufteilungsmethode darin, dass diese auch in Fällen angewendet werden kann, in denen keine vergleichbaren Geschäfte zwischen unabhängigen Unternehmen identifiziert werden können. Die Gewinnteilungsmethode bietet somit für solche Fälle Flexibilität, da für die Bestimmung des Gewinnaufteilungsmaßstabs angenommen wird, dass sich fremde Dritte unter den gegeben Umstände ebenso verhalten würden. In solchen Fällen beruht die Gewinnaufteilung auf der Funktionsteilung zwischen den verbundenen Unternehmen. Externe Daten von unabhängigen Unternehmen werden bei der Gewinnaufteilungsanalyse in erster Linie benötigt, um den Wert der Beiträge jedes verbundenen Unternehmens zu den Transaktionen zu beurteilen, und nicht um unmittelbar die Gewinnaufteilung vorzunehmen.

Schwierigkeiten bei der Anwendung der Gewinnaufteilungsmethode ergeben sich einerseits bei der Ermittlung des gemeinsamen Transaktionsgewinns, beispielsweise da nicht notwendigerweise der gesamte Unternehmensgewinn aufgeteilt werden muss bzw. weil es landesspezifische Unterschiede in der Ermittlung von Kosten und Umsätzen gibt. Zudem ist der Gewinnteilungsmaßstab oft schwierig zu substantiieren. Sofern kein

${ }^{31}$ Vgl. Tz. 2.111 bis 2.113 OECD-RL; Tz. 2.4.6. VWG 1983.

${ }^{32}$ Tz. 3.6 OECD-RL. 
Drittvergleich vorhanden ist (was in der Praxis oft der Fall ist), können mithilfe einer detaillierten Wertschöpfungsanalyse die jeweiligen Beiträge der beteiligten Parteien anhand der Funktionen, Risiken, eingesetzten Wirtschaftsgüter und Kosten ermittelt werden. Dies ist entweder auf quantitativer Basis möglich, sofern eine zuverlässige, beobachtbare Variable vorhanden ist, die signifikant mit dem eingebrachten Beitrag zur Wertschöpfung der beteiligten Parteien korreliert ist. Andererseits ist auch eine qualitative Analyse des relativen Wertschöpfungsbeitrags auf Basis von Expertenschätzungen/ Balanced Score Cards etc. denkbar.

Schließlich sei noch erwähnt, dass im Rahmen des OECD BEPS Projekts derzeit die Richtlinien zur Anwendung der Gewinnaufteilungsmethode derzeit überarbeitet werden. ${ }^{33}$ Insbesondere sollen noch weitere Anleitungen erarbeitet werden, in welchen Situationen die Gewinnaufteilungsmethode als Verrechnungspreismethode am besten geeignet ist, was unter hochintegrierten Geschäftsabläufen sowie einzigartigen und wertvolle Beiträgen zu verstehen ist, wie Synergien im Konzern zu behandeln sind, nach welchen Faktoren Gewinne zu teilen sind und inwiefern die Gewinnaufteilungsmethode verwendet werden kann, um Lizenzsätze für IWGs zu bestimmen oder die Resultate einer TNMM zu unterstützen. Zudem wird die Gewinnaufteilungsmethode von der OECD als einer der am besten geeignetsten Methoden zur Bestimmung von Verrechnungspreisen im Zusammenhang mit immateriellen Wirtschaftsgütern genannt (siehe weitere Ausführungen hierzu in Kap. 5.4.3.2 dieses Buches).

\subsubsection{Praktische Anwendung der Gewinnaufteilungsmethode}

Die Gewinnaufteilungsmethode wird insbesondere dann angewendet, wenn beide an der Transaktion beteiligten Unternehmen über maßgebliche immaterielle Wirtschaftsgüter verfügen, die den Gesamtgewinn aus der Transaktion stark beeinflussen und beide Parteien erhebliche wirtschaftliche Risiken tragen. Dies wäre beispielsweise der Fall wenn zwei oder mehrere verbundene Parteien wertvolle Patente und Know-how bzw. wertvolle Markenrechte in den Wertschöpfungsprozess einbringen. Unter diesen Bedingungen ist die Bestimmung fremdüblicher Preise oder Gewinnmargen aufgrund der Einzigartigkeit der eingesetzten immateriellen Wirtschaftsgüter nicht möglich. In diesem Fall wird deshalb auf der Basis einer Analyse des jeweiligen Wertschöpfungsbeitrags der beteiligten Parteien der Gesamtgewinn aufgeteilt.

Hinsichtlich der Ausgestaltung der angewendeten Gewinnaufteilung sind zahlreiche Varianten je nach untersuchtem Geschäftsmodell möglich. Man unterscheidet jedoch zwei grundsätzliche Anwendungsweisen des Profit Splits, die Beitragsanalyse („Contribution Profit Split“") und die Residualgewinnaufteilungsmethode, bzw. Restgewinnanalyse (,Residual Profit Split"). Beide Ausprägungen der Gewinnaufteilungsmethode sind in den Grafiken veranschaulicht (Abb. 5.6 und 5.7).

${ }^{33}$ Vgl. OECD (2015), Aligning Transfer Pricing Outcomes with Value Creation, Actions 8-10, S. $55-62$. 




Abb. 5.6 Beispiel Beitragsanalyse oder Contribution Profit Split



Abb. 5.7 Beispiel Residualgewinnaufteilungsmethode

Beim „Contribution Profit Split“ wird der aufzuteilende Gewinn (oder Verlust) zunächst ermittelt, in dem die Umsatzerlöse und Kosten aus der gemeinsamen Wertschöpfung gegenübergestellt werden. Wichtig in diesem Zusammenhang ist, dass nur der Gewinn oder Verlust aus den gemeinsamen Geschäft zu ermitteln ist und nicht notwendigerweise der gesamte Unternehmensgewinn der beiden Parteien. Sofern diese noch weitere Geschäftszweige haben, die nicht in Zusammenhang mit der gemeinsamen Transaktion stehen, ist der darin erwirtschaftete Gewinn oder Verlust nicht Bestandteil des Profit Splits.

Nach Ermittlung des Gesamtgewinns der beiden Parteien wird dieser nach einem fremdüblichen Schlüssel (z. B. typischerweise in dieser Industrie verwendete Parameter, falls vorhanden) bzw. auf Basis einer detaillierten Analyse des Wertbeitrags der jeweiligen Partei aufgeteilt. Als problematisch bei dieser Form des Profit Splits könnte sich erweisen, 
dass von einem der Parteien durchgeführte Routinefunktionen einen Verlust generieren könnten, da keine Vorabvergütung von gegebenenfalls vorhandenen Routinefunktionen erfolgt.

In der Verrechnungspreispraxis wird häufiger der „Residual Profit Split“ angewendet, wobei der Gruppengewinn aus der gemeinsamen Wertschöpfung nach Abzug von Routinevergütungen aufgeteilt wird. Die grobe Vorgehensweise ist in der folgenden Abb. 5.7 dargestellt.

Die typische Vorgehensweise bei der Anwendung eines Residual Profit Split ist wie folgt: Analog zur Beitragsanalyse ist in einem ersten Schritt der aufzuteilende Gewinn aus der gemeinsamen Geschäftsverbindung zu ermitteln. Hierzu ist es zunächst erforderlich, die relevanten externen Umsätze der beteiligten Parteien sowie die (Voll-) Kosten der jeweiligen Funktionen in der Wertschöpfungskette zu identifizieren. In einem nächsten Schritt wird analysiert, welche Funktionen als sogenannte Routine- und Nicht-Routine Funktionen eingestuft werden können. Den identifizierten Routine-Funktionen werden so dann jeweils Routinevergütungen zugeteilt. Diese werden in der Regel auf Basis der Transaktionsbezogenen Nettomargenmethode und den Ergebnissen von Datenbankstudien für die entsprechende Funktion ermittelt. Der Residual Profit Split ist somit eine Kombination verschiedener OECD-Verrechnungspreismethoden, nämlich der TNMM und der Gewinnaufteilungsmethode. Anschließend wird der Residualgewinn (oder -verlust) ermittelt, in dem die Vorabvergütungen für die Routinefunktionen vom Gesamtgewinn subtrahiert werden. Schließlich wird in einem letzten Schritt der Residualgewinn oder -verlust auf die identifizierten „Nicht-Routine Funktionen“ bzw. von den beteiligten Parteien eingebrachten, signifikanten immateriellen Wirtschaftsgütern aufgeteilt.

Die Bestimmung des Aufteilungsschlüssels ist in der Praxis häufig eine schwierige Fragestellung, da Informationen über Aufteilungsschlüssel aus Vergleichstransaktionen zwischen unverbundenen Parteien in der Regel nicht verfügbar sind. Insofern sollte der Aufteilungsschlüssel auf einer detaillierten Funktions- und Risikoanalyse sowie Werttreiberanalyse beruhen (,Value Chain Analysis“). Beispielsweise kann mit Hilfe von Interviews mit Experten aus den beteiligten Fachbereichen eine Gewichtung des relativen Beitrags der Funktionen der beteiligten Unternehmen ermittelt werden. Alternativ können sich quantitative Aufteilungsschlüssel wie z. B. Kosten, Lohnsumme, bilanziertes Vermögen usw. als geeignete Variable für den Wertbeitrag der beteiligten Unternehmen herausstellen. Die Voraussetzung für die Verteidigungsfähigkeit des ermittelten Schlüssels in einer Betriebsprüfung ist jedoch das Vorhandensein einer detaillierten Dokumentation, die objektiv darstellt, wie der angemessene Schlüssel ermittelt wurde.

Der Vorteil der Residualgewinnaufteilungsmethode ist, dass Routinefunktionen im Rahmen der gemeinsamen Wertschöpfungskette eine stabile, positive Vergütung erhalten. Die Nicht-Routine Funktionen, d. h. die unternehmerischen Funktionen, erhalten das Residualergebnis, welches positiv oder negativ sein kann. 


\section{Beispiel 5.6: Anwendung der Gewinnaufteilungsmethode}

\section{Sachverhalt:}

Die Pharma GmbH ist Teil eines internationalen Konzerns in der pharmazeutischen Industrie mit Sitz in den USA. Die Gesellschaft hat ein hochkomplexes technisches Verfahren zur Entwicklung von Grundsubstanzen für neue Medikamente entwickelt. Sobald eine Grundsubstanz für ein neues Medikament entwickelt wurde, übernimmt die Forschungs- und Entwicklungsabteilung der US Muttergesellschaft die Weiterentwicklung bis hin zum zulassungsfähigen Medikament und führt sämtliche klinischen Tests durch. Beide Gesellschaften tragen die Kosten der Entwicklung für ihren entsprechenden Teil der Entwicklung. Aus den gemeinsamen Entwicklungsarbeiten ging ein neues Medikament hervor, das sehr erfolgreich am Markt gestartet wurde. Das Medikament wird von der US Muttergesellschaft produziert und in den USA vermarktet. Die Pharma GmbH bezieht die produzierten Medikamente von der US Muttergesellschaft und vermarktet und vertreibt das Produkt in Europa.

Der Konzern steht nun vor der Frage, welcher Verrechnungspreis für die Lieferung der Medikamente der US Mutter an die deutsche Tochtergesellschaft anzusetzen ist, und wie die gemeinsame Forschungs- und Entwicklungsarbeit zu vergüten ist.

\section{Lösung:}

Der oben geschilderte Fall ist ein typisches Anwendungsbeispiel für die Gewinnaufteilungsmethode. Die OECD-Standardmethoden sind für den vorliegenden Fall nicht anwendbar, da es sich bei den gehandelten Medikamenten um einzigartige Produkte handelt, für die keine Fremdvergleichswerte (Preise oder Margen) verfügbar sind, zumal beide Gesellschaften ein wirtschaftliches und rechtliches Eigentum an dem Medikament haben. Beide Gesellschaften bringen einzigartige immaterielle Wirtschaftsgüter in die Wertschöpfung ein und tragen erhebliche wirtschaftliche Risiken (hier vor allem Forschungsrisiken). Somit haben beide Gesellschaften einen Anspruch auf das Residualergebnis aus der Vermarktung des Medikaments. Daher wird die Anwendung der Gewinnaufteilungsmethode als angemessen in diesem Fall betrachtet.

Da es keine zuverlässigen Fremdvergleichswerte für einen Gewinnaufteilungsschlüssel zwischen fremden Dritten Parteien in einem ähnlichen Fall gibt, wird die Residualgewinnaufteilungsmethode angewendet.

In einem ersten Schritt wird zunächst der gemeinsame Transaktionsgewinn der beiden Unternehmen aus dem Medikament auf Basis einer vorläufigen Gewinn- und Verlustrechnung ermittelt. Dies ist in Tab. 5.5 dargestellt.

Der aufzuteilende Gewinn aus dem gemeinsam entwickelten und vermarkteten Medikament beträgt EUR 220 Mio., wobei gemäß einer vorläufigen Gewinn- und Verlustrechnung die US Gesellschaft einen Gewinn von EUR 170 Mio. und die Pharma GmbH von EUR 50 Mio. erzielt.

In einem weiteren Schritt werden für die im Rahmen der Wertschöpfungskette ausgeübten Routinefunktionen Vorabvergütungen ermittelt. Die Funktions- und Risikoanalyse hat im vorliegenden Fall ergeben, dass die Funktionen Produktion, Laufende 
Tab. 5.5 Beispiel 5.6 Aufzuteilender Gewinn

\begin{tabular}{l|l|l}
\hline In Tausend EUR & US Corp. & Pharma GmbH \\
\hline Produktumsätze des Medikaments & 700.000 & 300.000 \\
\hline Davon Außenumsatz & 500.000 & 300.000 \\
\hline Davon Innenumsatz & 200.000 & 0 \\
\hline Wareneinsatz/Produktionskosten & 400.000 & 200.000 \\
\hline Laufende Entwicklungskosten & 20.000 & 10.000 \\
\hline Vertriebs- u. Gemeinkosten & 110.000 & 40.000 \\
\hline EBIT & 170.000 & 50.000 \\
\hline Aufzuteilender Gewinn & 220.000 & \\
\hline
\end{tabular}

Tab. 5.6 Beispiel 5.6 Ermittlung Routinegewinn

\begin{tabular}{|c|c|c|}
\hline & US Corp. & Pharma GmbH \\
\hline \multicolumn{3}{|l|}{ Routinevergütung Produktion } \\
\hline Produktionskosten & 400.000 & - \\
\hline Routinegewinn Produktion & 30.000 & - \\
\hline \multicolumn{3}{|c|}{ Routinevergütung Laufende Entwicklung } \\
\hline Kosten & 20.000 & 10.000 \\
\hline Routinegewinn Produktion & 2000 & 1000 \\
\hline \multicolumn{3}{|l|}{ Routinevergütung Vertrieb } \\
\hline Externe Umsätze & 500.000 & 300.000 \\
\hline Routinegewinn Vertrieb & 25.000 & 15.000 \\
\hline Summe Routinevergütung & 57.000 & 16.000 \\
\hline
\end{tabular}

Entwicklung und Vertrieb als Routinefunktion eingestuft werden können, wohingegen die Funktionen Forschung \& Entwicklung des Medikaments auf Basis von einzigartigem Know-how als die werttreibende „Nicht-Routine“ Funktion identifiziert wird. Auf Basis von durchgeführten Datenbankstudien wurden folgende Routinevergütungen ermittelt:

Routinevergütung Produktion: Gewinnaufschlag von 7,5\% auf die Kosten

Routinevergütung Laufende Entwicklung: Gewinnaufschlag von $10 \%$ auf die Kosten

Routinevergütung Vertrieb: Rendite auf den Umsatz von 5\%

Anhand dieser Werte werden die Routinevergütungen berechnet, siehe Abb. 5.6 (Tab. 5.6).

Schließlich müssen in einem letzten Schritt der Residualgewinn und der anzuwendende Aufteilungsschlüssel bestimmt werden. Der aus der gemeinsamen Geschäftstransaktion resultierende Residualgewinn ist nach dem jeweiligen Beitrag zur Wertschöpfung und insbesondere dem Beitrag von einzigartigen immateriellen 
Tab. 5.7 Beispiel 5.6 Aufteilung Residualgewinn

\begin{tabular}{l|l|l}
\hline & US Corp. & Pharma GmbH \\
\hline Aufzuteilender Gewinn & 220.000 & \\
\hline Summe Routinevergütungen & 73.000 & \\
\hline Residualgewinn & 147.000 & \\
\hline Anteil Residualgewinn $(2 / 3: 1 / 3)$ & 98.000 & 49.000 \\
\hline Gesamtgewinn & 155.000 & 65.000 \\
\hline Vorläufiger Gewinn & 170.000 & 50.000 \\
\hline Ausgleichszahlung & -15.000 & 15.000 \\
\hline
\end{tabular}

Wirtschaftsgütern (Technologie, Patente auf Medikament) zwischen den Parteien aufzuteilen. Eine detaillierte Analyse der Beiträge zur Entwicklung des Medikaments, der über den Zeitraum der Forschung und Entwicklung getragenen Kosten und Risiken auf Basis von Experteninterviews hat ergeben, dass ein Aufteilungsschlüssel von zwei Drittel (US Corp.) zu einem Drittel (Pharma GmbH) angemessen ist.

Entsprechend wird nun der Residualgewinn ermittelt und anhand des Aufteilungsschlüssels auf die beiden Parteien verteilt. Schließlich muss noch bestimmt werden, ob gegebenenfalls Ausgleichszahlungen erforderlich sind, damit die Parteien den fremdüblichen Gewinn gemäß des Residual Profit Splits erhalten (Tab. 5.7).

Als Ergebnis des vorliegenden Profit Splits erhalten die US Corp. einen Residualgewinn von ca. EUR 98 Mio. und die Pharma GmbH einen Residualgewinn von EUR 49 Mio. Hinzu kommt noch der jeweilige Anteil an den Routinevergütungen, sodass ein Gesamtgewinn von EUR 155 Mio. für die US Corp. und EUR 65 Mio. für die Pharma GmbH fremdüblich wären. Da die US Corp. auf Basis der vorläufigen Gewinn- und Verlustrechnung einen höheren Gewinn aufweist, als gemäß Profit Split Analyse angemessen wäre, muss sie eine Ausgleichszahlung von EUR 15 Mio. an die Pharma GmbH leisten.

Während in dem obigen Beispiel ein einzelner Betrag für die jeweiligen Gewinnanteile der am Profit Split beteiligten Unternehmen angegeben wird, ist es in der Praxis zur Vereinfachung ggf. möglich, eine Bandbreite von Gewinnanteilen durch Ausnutzung der fremdüblichen Bandbreite von Routinevergütungen herzuleiten. Der Vorteil dieser Herangehensweise ist, dass dadurch die Höhe der Ausgleichszahlungen am Jahresende verringert werden kann, da nicht auf einen einzelnen Gewinnbetrag angepasst wird sondern lediglich in einem Umfang, dass alle Parteien einen Gewinn innerhalb der fremdüblichen Bandbreite von Gewinnanteilen erzielen. 


\subsection{Besonderheiten hinsichtlich der Verrechnungspreismethoden in Deutschland}

\subsubsection{Methodenhierarchie}

Im vorangegangenen Abschnitt wurden die OECD-Verrechnungspreismethoden vorgestellt und auf ihre Anwendbarkeit in der Verrechnungspreispraxis eingegangen. Gemäß OECD-RL sollte die Auswahl der Verrechnungspreismethode jeweils darauf abzielen, die vor dem Hintergrund der Verfügbarkeit von Fremdvergleichsdaten, der jeweiligen Transaktionsart und Funktionsanalyse am besten geeignete Methode zu wählen. ${ }^{34}$ Zwar bezeichnen die OECD-RL die geschäftsvorfallbezogenen Standardmethoden als die Methoden, mit denen sich am unmittelbarsten feststellen lässt, ob die konzerninternen Transaktionen fremdüblichen Bedingungen unterliegen. ${ }^{35}$ Allerdings wird auch festgestellt, dass die Standardmethoden in der Praxis aufgrund mangelnder empirischer Fremdvergleichswerte weniger geeignet sein können als die gewinnbezogenen Methoden.

Mit der Unternehmenssteuerreform 2008 und Änderung des $\S 1$ Abs. 3 AStG zum 1.1.2008 wurde in Deutschland eine gesetzlich vorgeschriebene Hierarchie von Verrechnungspreismethoden eingeführt, die der Steuerpflichtige bei der Wahl der Verrechnungspreismethode zu berücksichtigen hat. Gemäß der gesetzlichen Regelung ist die Auswahl der angemessenen Verrechnungspreismethode insbesondere von der Verfügbarkeit geeigneter Fremdvergleichsdaten abhängig. So soll der Steuerpflichtige vorrangig die OECDStandardmethoden, d. h. die Preisvergleichs-, die Wiederverkaufspreis- oder die Kostenaufschlagsmethode anwenden, ,wenn Fremdvergleichswerte ermittelt werden können, die nach Vornahme sachgerechter Anpassungen im Hinblick auf die ausgeübten Funktionen, die eingesetzten Wirtschaftsgüter und die übernommenen Chancen und Risiken (Funkti-


schränkt vergleichbare Werte ermittelt werden können, bilden diese eine Bandbreite. In diesen Fällen darf der Steuerpflichtige die Bandbreite voll ausnutzen.

Wenn keine uneingeschränkt vergleichbaren Werte ermittelt werden können, aber eingeschränkt vergleichbare Werte nach Vornahme sachgerechter Anpassungen vorliegen, sind diese der Anwendung einer geeigneten Verrechnungspreismethode zugrunde zu legen, d. h., grundsätzlich sind alle OECD-Verrechnungspreismethoden inklusive der Gewinnmethoden zulässig. In diesem Fall ist die resultierende Bandbreite einzuengen, etwa durch Anwendung des Konzepts der interquartilen Bandbreite. ${ }^{37}$ Falls auch keine eingeschränkt vergleichbaren Fremdvergleichswerte festgestellt werden können, hat der Steuerpflichtige einen hypothetischen Fremdvergleich durchzuführen. ${ }^{38}$ Beim hypothetischen

\footnotetext{
${ }^{34}$ Tz. 2.2 OECD-RL.

${ }^{35} \mathrm{Vgl}$. Tz. 2.3 OECD-RL.

${ }^{36}$ Vgl. $§ 1$ Abs. 3 S. 1 AStG.

${ }^{37}$ Vgl. $§ 1$ Abs. 3 S. 2 und S. 3 AStG.

${ }^{38}$ Vgl. $§ 1$ Abs. 3 S. 5 AStG.
} 
Fremdvergleich wird auf Basis einer hypothetischen Analyse der Zahlungsbereitschaft des Käufers der Leistung (Höchstpreis) und dem minimalen akzeptablen Preis aus Sicht des Verkäufers (Mindestpreis) ein Einigungsintervall aufgestellt. Der fremdübliche Verrechnungspreis soll sich dann innerhalb dieses Intervalls befinden, wobei der wahrscheinlichste Einigungspunkt (oder wenn kein anderer Wert glaubhaft gemacht werden kann) der Mittelwert als Verrechnungspreis gewählt werden soll. Im Rahmen seiner Aufzeichnungspflichten hat der Steuerpflichtige in Deutschland die Wahl der verwendeten Verrechnungspreismethode zu begründen. d. h., es muss auch dokumentiert werden, ob uneingeschränkt vergleichbare, eingeschränkt vergleichbare oder überhaupt keine Fremdvergleichswerte vorliegen.

Abbildung 5.8 gibt die Methodenhierarchie in Deutschland gemäß $\S 1$ Abs. 3 AStG grafisch wieder.

In der Verrechnungspreispraxis wird man häufig schnell feststellen, dass uneingeschränkt vergleichbare Fremdvergleichswerte für konzerninterne Transaktionen nicht identifiziert werden können. Fremdvergleichswerte aus Datenbanken können nicht als uneingeschränkt vergleichbar bezeichnet werden, da die vorliegenden Informationen zu den potenziellen Vergleichsunternehmen begrenzt sind und häufig nicht alle Umstände genau auf Vergleichbarkeit geprüft werden können. Um uneingeschränkt vergleichbare Fremdvergleichswerte zu identifizieren, müsste der Steuerpflichtige im Prinzip beinahe die gleiche Transaktion unter identischen Bedingungen mit fremden Dritten unterhalten. Da dies selten der Fall ist und in der Praxis zumeist eingeschränkt vergleichbare Werte identifiziert werden können, ist in der Folge zumeist jede geeignete Verrechnungspreismethode anwendbar. Zu beachten ist dann jedoch, dass nicht die volle Bandbreite der eingeschränkt



Abb. 5.8 Methodenhierarchie in Deutschland gemäß $\S 1$ Abs. 3 AStG 
vergleichbaren Werte angewendet werden kann, sondern die Bandbreite mittels eines geeigneten Ansatzes einzuschränken ist.

Es gibt jedoch auch Situationen, in denen noch nicht einmal eingeschränkt vergleichbare Werte vorliegen. In der Regel sind dies Fälle von Funktionsverlagerungen, aber auch Transaktionen in sehr speziellen Industrien mit wenigen Marktteilnehmern, für die es äußerst schwierig ist, geeignete Daten für den Fremdvergleich zu identifizieren. In der Betriebsprüfungspraxis lässt sich jedoch beobachten, dass die deutsche Finanzverwaltung den hypothetischen Fremdvergleich durchaus auch häufig für andere Transaktionen anwenden möchte, insbesondere wenn der Nachweis der Fremdüblichkeit auf Basis von eingeschränkt vergleichbaren Werten aus Datenbanken zu einer aus ihrer Sicht nicht gewünschten Gewinnverteilung führt. Der hypothetische Fremdvergleich wird im nachfolgenden Kapitel ausführlicher behandelt.

\subsubsection{Hypothetischer Fremdvergleich und Funktionsverlagerung}

Der hypothetische Fremdvergleich als Verrechnungspreismethode kann als ein Sonderfall der deutschen Verrechnungspreisvorschriften bezeichnet werden. Wie in Kap. 5.1 ausgeführt, war der Zweck der OECD-RL einen einheitlichen Rahmen und gewisse Rechtssicherheit bei der Anwendung des Fremdvergleichsgrundsatzes in verschiedenen Ländern zu schaffen, um auf diesem Weg das Risiko von Doppelbesteuerung in internationalen konzerninternen Transaktionen zu verringern. Der hypothetische Fremdvergleich ist ein Sonderweg, der nicht unbedingt zur Rechtssicherheit für den Steuerpflichtigen beiträgt, da er hinsichtlich des Fremdvergleichs auf ein hypothetisches Denkmodell und nicht auf Fremdvergleichsdaten, seien diese auch nur eingeschränkt vergleichbar, abstellt. Da es sich bei Verrechnungspreisfragen in den meisten Fällen um internationale Sachverhalte handelt, sollte daher von der Anwendung dieses deutschen Sonderwegs „sparsam“ Gebrauch gemacht werden, auch zumal immer das Risiko besteht, dass die deutsche Finanzverwaltung ein anderes „Denkmodell“ verfolgen kann. Insofern sollte bei der ökonomischen Verrechnungspreisanalyse jeweils genau geprüft werden, ob nicht zumindest eingeschränkt vergleichbare Fremddaten vorliegen, etwa durch Anwendung weniger restriktiver Annahmen (etwa hinsichtlich Produkte, Industriezweig) bei der Suche nach unabhängigen Vergleichsunternehmen oder ob die Gewinnaufteilungsmethode in Betracht kommt. Auch die OECD äußert mittlerweile eine Präferenz für zweiseitige Verrechnungspreisansätze. Insbesondere sollten laut OECD bei der Verrechnungspreisbestimmung die realistischen Handlungsalternativen der beteiligten Unternehmenseinheiten berücksichtigt werden. Daher ist zu erwarten, dass die generelle Vorgehensweise beim hypothetischen Fremdvergleich in der Praxis zukünftig stark zunehmen wird.

Im Falle der Anwendung des hypothetischen Fremdvergleichs auf reguläre Geschäftsbeziehungen zwischen verbundenen Unternehmen wird der Mindestpreis des Leistenden dem Wert entsprechen, bei dem sich das Geschäft für den Leistenden noch rentiert. Dies wird in vielen Fällen den Kosten (in bestimmten Konstellationen auch Grenzkosten) der Leistung entsprechen, da ein unabhängiger Marktteilnehmer in aller Regel 
Gewinnerzielungsabsichten verfolgt und mindestens seine Kosten decken bzw. einen Gewinn erzielen möchte. In Abhängigkeit von der verfolgten Marktstrategie des Konzerns und der Verteilung der Funktionen und Risiken zwischen den Konzernunternehmen können in bestimmten Einzelfällen aber auch für eine gewisse Zeit Preise unterhalb der Kosten denkbar sein, beispielsweise im Falle von Marktverdrängungsstrategien und wenn signifikante Gewinnpotentiale für den Leistungserbringer in der Zukunft erwartet werden. Dies ist abhängig von der hypothetischen Verteilung der Geschäftsrisiken und Chancen und sollte bei einer solchen Konstellation im Rahmen einer Betriebsprüfung auch glaubhaft gemacht werden können. Desweiteren kann der Mindestpreis ebenfalls durch eine Kapitalbudgetierungsanalyse anhand der geplanten Cash Flows über die Laufzeit des Geschäfts und unter Berücksichtigung der Kapitalkosten bestimmt werden. Der Mindestpreis würde dann dem Preis entsprechen, bei dem der Barwert des Geschäfts für den Leistenden bei Vereinbarung des Geschäfts mindestens positiv ist bzw. größer als der Barwert möglicher weiterer Geschäftsfelder, in die der Leistende investieren kann.

Aus Sicht des Käufers der Leistung ist die Betrachtung ähnlich wie beim Verkäufer, da dieser in den meisten Fällen die Leistung nur kaufen würde, wenn er insgesamt aus dem Geschäft seine Kosten decken bzw. einen geringen Gewinn erzielen würde. Der Höchstpreis würde dann in der Regel dem Preis entsprechen, bei dem der Gewinn mindestens null entspricht, bzw. bei dem der Barwert des Geschäfts bei Vereinbarung des Geschäfts insgesamt positiv ist und keine besseren Investitionsalternativen zur Verfügung stehen.

Aus dem ermittelten Mindestpreis des Verkäufers und dem Höchstpreis des Käufers sollte in aller Regel ein Einigungsintervall resultieren, da für die ökonomische Verrechnungspreisanalyse angenommen wird, dass die Transaktionen betriebswirtschaftlichen Grundsätzen entsprechen. Sofern der Höchstpreis des Käufers unterhalb des Mindestpreises des Verkäufers liegt, würde im Prinzip keine Einigung zustande kommen. Dies würde bedeuten, dass die Transaktion aus Konzernsicht im Prinzip nicht betriebswirtschaftlich sinnvoll ist bzw. einige der getroffenen Annahmen sind gegebenenfalls zu überprüfen. Innerhalb des Einigungsintervalls soll der Preis festgelegt werden, welche mit höchster Wahrscheinlichkeit dem Fremdvergleichsgrundsatz entspricht. Kann kein Wert glaubhaft gemacht werden, ist der Mittelwert zugrunde zu legen.

Der methodische Ansatz ist in Abb. 5.9 grafisch veranschaulicht.

Sofern das Einigungsintervall bestimmt wurde, besteht die Schwierigkeit analog zur Gewinnaufteilungsmethode den für beide Parteien angemessenen Aufteilungsschlüssel bzw. Einigungswert zu bestimmen. Im Prinzip ist der hypothetische Fremdvergleich ein Sonderfall der Gewinnaufteilungsmethode, da das Einigungsintervall in der Regel zwischen den Werten verläuft, bei denen der gesamte Gewinn aus der Transaktion auf den Käufer (Mindestpreis) oder auf den Verkäufer (Höchstpreis) entfällt. Für die Ermittlung des Einigungswertes kommen entweder empirische Vergleichswerte infrage (beispielsweise sofern Informationen über Gewinnaufteilungen unter fremden Dritten wie etwa Joint Ventures vorhanden sind), eine spieltheoretische Betrachtung (beispielsweise wie wäre die Verhandlungsmacht bzw. die Handlungsalternativen der jeweiligen Vertragspartner unter fremdüblichen Bedingungen) oder ein Aufteilungsschlüssel auf Basis einer qualitativen Analyse der jeweiligen Funktionen, Risiken und Wertschöpfungsbeiträge der 


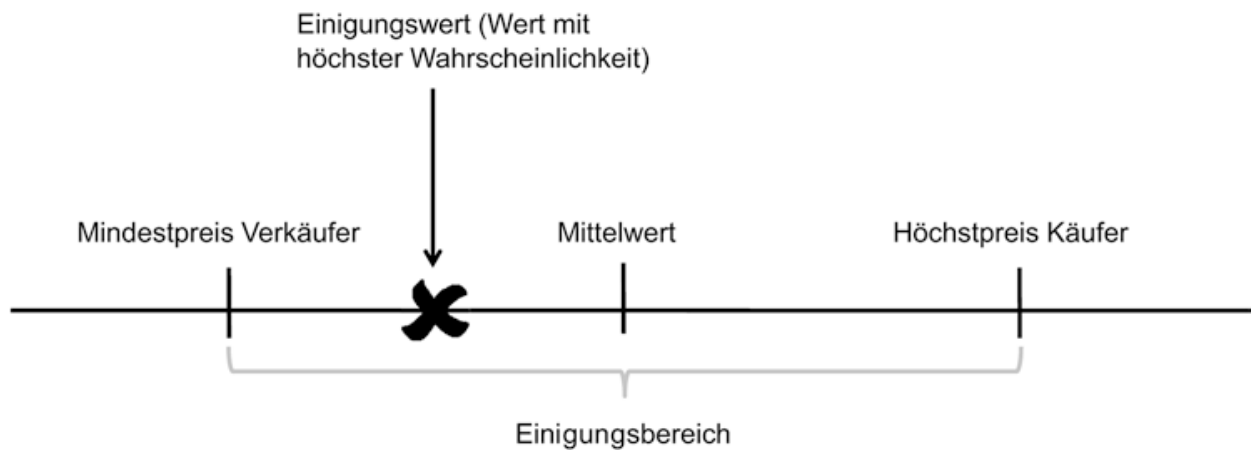

Abb. 5.9 Hypothetischer Fremdvergleich

Transaktionspartner. Die Verwendung des Mittelwerts ohne zugrunde liegende Analyse würde vermutlich vom Fremdvergleichsgrundsatz abweichen, da es im Prinzip keine ökonomische Fundierung gibt, warum dieser Wert angemessen sein soll.

Im Falle von Funktionsverlagerungen (vgl. Ausführungen hierzu in Abschn. 2.2.4.3 dieses Buches) ist, sofern keine der Ausnahmeregelungen (,Escape Klauseln“) des $\S 1$ Abs. 3 AStG bzw. der FVerlV anwendbar ist, in aller Regel davon auszugehen, dass der Verrechnungspreis für die verlagerte Funktion inklusive der damit verbundenen Wirtschaftsgüter, Chance und Risiken sowie sonstigen Vorteilen als Ganzes (sog. Transferpaket) auf Basis des hypothetischen Fremdvergleichs zu bestimmen ist. In den meisten Fällen werden aufgrund der Einzigartigkeit der im Rahmen von Funktionsverlagerungen transferierten Wirtschaftsgüter in der Regel weder uneingeschränkt noch eingeschränkt vergleichbare Fremdvergleichsdaten für das Transferpaket vorliegen, sodass keine der OECD-Verrechnungspreismethoden zuverlässig anwendbar wäre. Somit erscheint lediglich die Preisvergleichsmethode als eine sinnvolle Alternative zum hypothetischen Fremdvergleich bei der Bestimmung eines Verrechnungspreises für das Transferpaket. Dies könnte beispielsweise der Fall sein, wenn ein ähnlicher Teilbetrieb oder Betriebszweig im Konzern kurz zuvor bereits an einen fremden Dritten veräußert wurde. Dann könnten anhand der dort vereinbarten Preise Rückschlüsse auf den Wert der konzernintern transferierten Funktion möglich sein, ggf. unter Durchführung von Anpassungsrechnungen hinsichtlich der Größe des Geschäfts.

Die Anwendung des hypothetischen Fremdvergleichs im Fall von Funktionsverlagerungen wird im folgenden Abschnitt kurz umrissen (detaillierte Anwendungsvorschriften sind in der Funktionsverlagerungsverordnung sowie im BMF-Schreiben zur Funktionsverlagerung enthalten). Vom Grundsatz her sind zur Bestimmung des Einigungsbereichs vier Bewertungen erforderlich:

1. Bewertung Gewinnpotential des Funktion abgebenden Unternehmens vor der Funktionsverlagerung

2. Bewertung Gewinnpotential des Funktion abgebenden Unternehmens nach der Funktionsverlagerung 
3. Bewertung Gewinnpotential des Funktion aufnehmenden Unternehmens vor der Funktionsverlagerung

4. Bewertung Gewinnpotential des Funktion aufnehmenden Unternehmens nach der Funktionsverlagerung

In der Praxis kann es jedoch auch genügen lediglich zwei vollständige Bewertungen durchzuführen, da oftmals davon auszugehen ist, dass der Wert der in Betracht stehenden Funktion des abgebenden Unternehmens nach der Verlagerung gleich null ist bzw. den Schließungskosten entspricht. Gleichsam wird in der Regel der Wert der Funktion aus Sicht des aufnehmenden Unternehmens vor der Verlagerung ebenfalls null sein. Jedoch wäre es hierzu erforderlich, segmentierte Finanzzahlen für die zu verlagernde Funktion zu ermitteln.

Die Bewertung hat auf Grundlage der betrieblichen Plandaten (budgetierte Cash Flows bzw. Reingewinne nach Steuern) hinsichtlich der Funktion zu erfolgen, welche die Grundlage für die Entscheidung der Funktionsverlagerung gebildet haben. Dabei sollen potentielle Synergien sowie Standortvorteile des aufnehmenden Unternehmens berücksichtigt werden. Darüber hinaus ist ein der Funktion angemessener Kapitalisierungszeitraum für die Bewertung zugrunde zu legen. Wenn keine Gründe für einen bestimmten Zeitraum glaubhaft gemacht werden können, ist gemäß Funktionsverlagerungsverordnung (vgl. § 6 FVerlV) von einem unbegrenzten Zeitraum auszugehen. Diese Annahme ist in der Praxis genau zu untersuchen, da die Annahme eines unendlichen Kapitalisierungszeitraums einen erheblichen Einfluss auf den Wert der Funktion haben kann. Der jeweils für die Bewertung anzunehmende Kapitalisierungszinssatz soll sich nach dem laufzeitspezifischen risikolosen Zinssatz zuzüglich eines funktions- und risikoadäquaten Zuschlags richten. Schließlich soll das übertragene Gewinnpotential beider Parteien auf Basis des Reingewinns nach Steuern bestimmt werden.

Auf Basis der Bewertungen wird dann zwischen dem entgangenen Gewinnpotential des abgebenden Unternehmens (Mindestpreis) sowie dem erhaltenen Gewinnpotential des aufnehmenden Unternehmens (Höchstpreis) das Einigungsintervall aufgestellt. Dies ist in Abb. 5.10 grafisch dargestellt.

Im Allgemeinen wird davon ausgegangen, dass der Höchstpreis des Empfängers über der minimalen Kaufpreisforderung des abgebenden Unternehmens liegt, andernfalls würden sich beide Unternehmen konsolidiert betrachtet aufgrund der Verlagerung schlechter stellen, d. h., die Sinnhaftigkeit der Verlagerung wäre aus betriebswirtschaftlicher Sicht anzuzweifeln.

Im Falle der Verlagerung einer verlusttragenden Funktion können sich auch ein negativer Mindest- und Höchstpreis ergeben. Dies würde bedeuten, dass das übertragende Unternehmen dem Aufnehmenden eine Ausgleichszahlung für die Übernahme der Funktion erstattet. Allerdings dürfte der Mindestpreis nicht unterhalb der Schließungskosten des abgebenden Unternehmens liegen, da es aus Sicht des abgebenden Unternehmens günstiger wäre, die Funktion einzustellen, als weiter fortzuführen. 


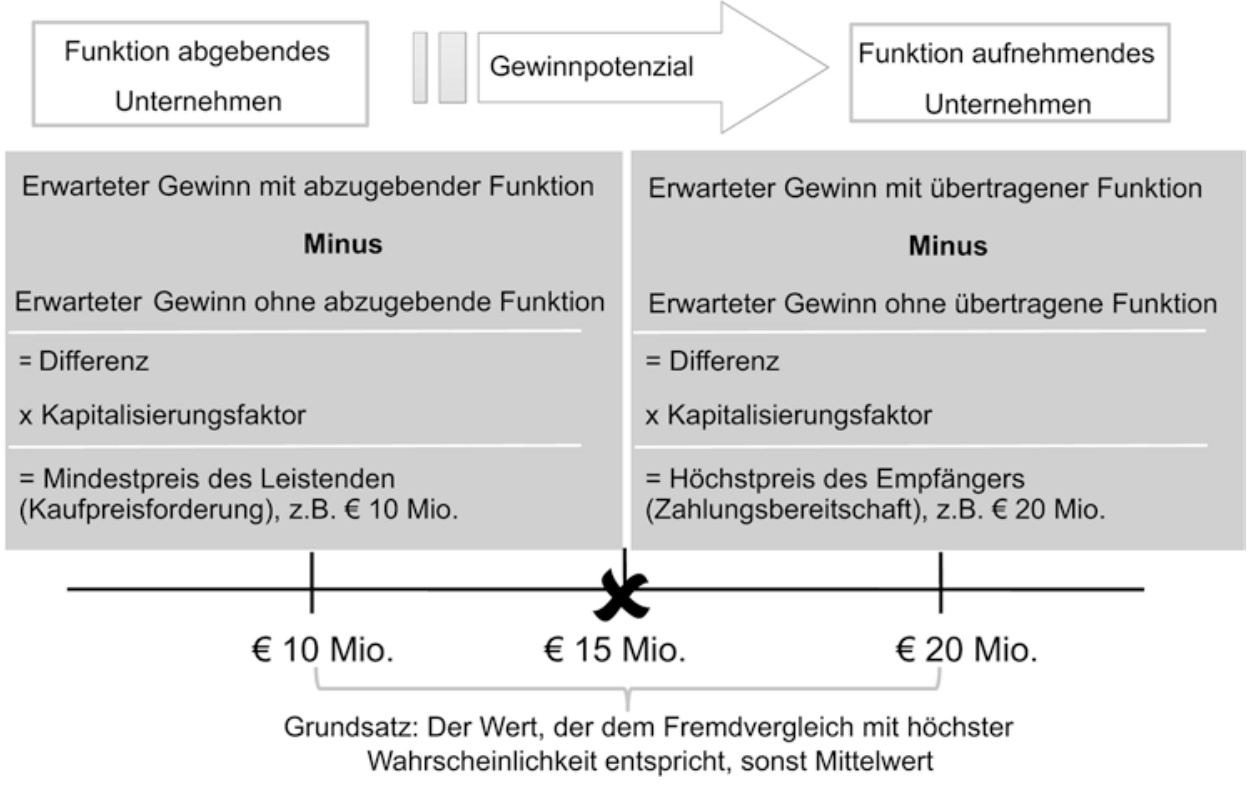

Abb. 5.10 Beispiel hypothetischer Fremdvergleich bei Funktionsverlagerung

Als Einigungswert ist der Wert anzusetzen, der mit höchster Wahrscheinlichkeit dem Fremdvergleichsgrundsatz entspricht. Sofern nichts anderes glaubhaft gemacht werden kann, ist der Mittelwert anzusetzen.

Als allgemein sehr kritisch gesehen wird, neben der äußerst breiten Definition von Funktionsverlagerungsfällen, dass die deutsche Steuergesetzgebung hinsichtlich des hypothetischen Fremdvergleichs von den OECD-RL abweicht. Die beidseitige Betrachtung der Gewinnpotentiale bei der Ermittlung des Einigungsbereichs kann insbesondere dazu führen, dass Synergien, Standort- oder Kostenvorteile sowie Steuervorteile des aufnehmenden Unternehmens im Ausland in die Verrechnungspreisbestimmung einfließen, die dann über die vereinbarte Ausgleichszahlung der Besteuerung in Deutschland unterliegen können. Es ist zwar auch in der betriebswirtschaftlichen Praxis unter fremden Dritten davon auszugehen, dass der Verkäufer versucht die Zahlungsbereitschaft des Käufers zu antizipieren, jedoch liegen keinesfalls vollständige Informationen insbesondere hinsichtlich der Standort- und Kostenvorteilen oder unternehmensspezifischen Steuersätzen des Käufers vor, welche die Entscheidung des Verkäufers beeinflussen können. Eine solche vollständige Transparenz wird jedoch in den deutschen Verrechnungspreisvorschriften (insbesondere $\S 1$ Abs. $1 \mathrm{AStG}$ ) als Grundannahme unterstellt. Insofern stellt dies im Prinzip eine Abweichung vom Fremdvergleichsgrundsatz dar. Schließlich ist noch die Anpassungsregelung hinsichtlich des vereinbarten Preises für die Funktion zu nennen (vgl. $\S 1$ Abs. 3 Satz 11 und 12, $\S 10,11$ FVerlV), welche vorsieht, dass bei einer erheblichen Abweichung der Gewinnentwicklung innerhalb von 10 Jahren nach der Funktionsverlagerung eine Anpassung auf den Verrechnungspreis vorzunehmen wäre, da unabhängige 
Dritte bei großer Unsicherheit eine solche Anpassungsklausel vereinbaren würden. Eine erhebliche Abweichung wäre demnach gegeben, wenn der auf Basis der tatsächlichen Gewinnentwicklung ermittelte Preis außerhalb des ursprünglichen Einigungsintervalls liegt bzw. der neu ermittelte Höchstpreis unterhalb des ursprünglichen Mindestpreises. Die Vereinbarung einer solchen Anpassungsregelung unter fremden Dritten erscheint jedoch als nicht unbedingt geschäftsüblich und wird selten vereinbart, zumal der Zeitraum der Anpassung über zehn Jahre als sehr lang erscheint. ${ }^{39}$ In der Verrechnungspreispraxis werden von Steuerpflichtigen im Falle von Funktionsverlagerungen daher häufig von den o. g. gesetzlichen Regeln abweichende Anpassungsklauseln mit einem kurzen Zeitraum für Preisanpassungen vereinbart. Im Rahmen des BEPS Projekts hat die OECD nun ähnliche ,ex-post“" Anpassungsregelungen für sog. schwer zu bewertende IWG veröffentlicht (siehe nähere Ausführungen in Abschn. 5.4.3.2 dieses Buches), bei denen die Vorhersage von zukünftigen Cash Flows zum Zeitpunkt der Übertragung ebenso mit großer Unsicherheit behaftet ist.

\subsection{Besondere Methoden für spezifische Transaktionen}

\subsubsection{Dienstleistungen im Konzern}

\subsubsection{Verrechnung von Dienstleistungen dem Grunde nach}

Die Verrechnung von Dienstleistungen innerhalb von Konzernen (insbesondere sogenannte Management Services, die von der Muttergesellschaft bzw. von konzerninternen Servicecentern erbracht werden) ist ein häufig geprüftes Thema im Rahmen von Betriebsprüfungen in Deutschland und vielen anderen Ländern. Im Vordergrund der Prüfung steht dabei zunächst, ob die Verrechnung der erbrachten Leistung an ein verbundenes Unternehmen dem Grunde nach gerechtfertigt ist. Dies ist der Fall, wenn tatsächlich eine Leistung an ein verbundenes Unternehmen erbracht wurde, und dem empfangenden Unternehmen daraus ein ökonomischer Nutzen entstanden ist. Ein wirtschaftlicher Nutzen würde grundsätzlich dann bestehen, wenn das empfangende Unternehmen diese Leistung sonst für sich selbst erbringen müsste (beispielsweise durch Einstellen neuer Mitarbeiter) oder diese Leistung anderweitig einkaufen müsste. ${ }^{40}$ Diese beiden Aspekte sind im Rahmen der ökonomischen Analyse zu prüfen, und dann im Rahmen der Verrechnungspreisdokumentation entsprechend zu dokumentieren.

Die Prüfung des wirtschaftlichen Nutzens des Empfängers ist hier von großer Bedeutung, denn es gibt eine Reihe von betrieblichen Tätigkeiten und Funktionen, die für verbundene Unternehmen ausgeübt werden, obwohl die empfangenden Unternehmen

${ }^{39}$ Vgl. hierzu auch A. Ebering, ,Wann sind Preisanpassungen bei Funktionsverlagerungen i. S. v. $\S 1$ Abs. 3 Satz 9 AStG fremdüblich?“, IStR 11/2011, S. 418 ff.

${ }^{40}$ Vgl. hierzu Tz. 7.6 OECD (2015), Aligning Transfer Pricing Outcomes with Value Creation, Actions 8-10. 
die Tätigkeit für sich betrachtet nicht benötigen und für diese kein Entgelt zahlen würden, wenn sie unabhängige Parteien wären. Dies ist häufig im Rahmen von Management Dienstleistungen der Fall, wenn bestimmte innerbetriebliche Aufgaben, welche ein Unternehmen der Gruppe (üblicherweise die Muttergesellschaft oder eine regionale Holdinggesellschaft) ausschließlich aufgrund ihrer Beteiligung an einer oder mehrerer der Gruppengesellschaften ausübt, also in der Funktion als Gesellschafter. Da kein direkter wirtschaftlicher Nutzen für den vermeintlichen Leistungsempfänger entsteht, würde eine solche Aktivität keine Weiterbelastung zum Empfänger rechtfertigen. Entsprechend den OECD-RL ${ }^{41}$ wird eine solche Aktivität als Gesellschafteraufwand bezeichnet. Die OECD nennen hierzu die folgenden Beispiele für sog. Shareholderkosten:

- Kosten für Aktivitäten in Zusammenhang mit der rechtlichen Struktur des Konzerns (beispielsweise Shareholder Meetings, Kosten des Aufsichtsrats, Kosten für die Ausgabe von Aktien),

- Kosten für die Erstellung von konsolidierten (Konzern-) Abschlüssen bzw. sonstige Mitteilungspflichten,

- Kosten für die Finanzierung von Unternehmensakquisitionen,

- Kosten für die Erfüllung der steuerlichen Mitwirkungspflichten der Muttergesellschaft,

- Kosten, die bei der Muttergesellschaft bezüglich der Einhaltung der Grundsätze ordentlicher Unternehmensführung (Corporate Governance) des Gesamtkonzerns anfallen.

Demgegenüber legt die deutsche Finanzverwaltung den Begriff des Gesellschafteraufwands wesentlich weiter aus. In den VWG 1983 wird eine nicht abschließende Liste von Gesellschafteraktivitäten genannt, für welche keine Entgelte verrechnet werden dürfen (siehe Tz. 6.3.2). Dies beinhaltet:

- den sogenannten Rückhalt im Konzern einschließlich des Rechts, den Konzernnamen zu führen, sowie der Vorteile, die sich allein aus der rechtlichen, finanziellen und organisatorischen Eingliederung in den Konzern ergeben;

- die Tätigkeit ihres Vorstandes und Aufsichtsrates als solche sowie für ihre Gesellschafterversammlungen;

- die rechtliche Organisation des Konzerns als ganzen sowie für die Produktions- und Investitionssteuerung im Gesamtkonzern;

- Tätigkeiten, die Ausfluss ihrer Gesellschafterstellung sind, einschließlich der allgemeinen Organisation sowie der der Konzernspitze dienenden Kontrolle und Revision;

- Schutz und Verwaltung der Beteiligungen;

- die Konzernführung und solche Führungsaufgaben nachgeordneter Unternehmen, die die Konzernspitze an sich gezogen hat, um ihre eigenen Führungsmaßnahmen besser vorzubereiten, durchzusetzen und zu kontrollieren. Die Führung schließt die Planung, die unternehmerische Entscheidung und die Koordinierung ein.

${ }^{41}$ Vgl. Tz. 7.9 ff. ebenda. 
Sofern Dienstleistungen durch eine ausländische Muttergesellschaft an deutsche verbundene Unternehmen verrechnet werden, sollte geprüft werden, dass Gesellschafteraktivitäten nicht an die deutsche Gesellschaft fakturiert werden, da dies von der deutschen Finanzverwaltung mit der Begründung aufgegriffen werden kann, es handele sich nicht um Leistungen, die einen wirtschaftlichen Nutzen für die deutsche Gesellschaft haben. Im umgekehrten Fall, d. h. der Verrechnung von Leistungen einer deutschen Muttergesellschaft an ausländische verbundene Unternehmen, dürften diese analog auch nicht weiterverrechnet werden. Allerdings wird in diesem Fall der Fokus der Prüfung durch die deutsche Finanzverwaltung darauf liegen, dass die Kosten für sämtliche Aktivitäten zum Nutzen der Auslandsgesellschaften verrechnet wurden.

Die oben erläuterte Problematik der Abgrenzung von Gesellschafteraktivitäten von Aktivitäten zum wirtschaftlichen Vorteil anderer verbundenen Unternehmen stellt sich häufig im Rahmen von zentralisierten Dienstleistungen der Konzernmutter. Grundsätzlich sind durch die Konzernmutter erbrachte Leistungen, sofern sie nicht unter Gesellschafteraktivitäten fallen, verrechenbar. Dies betrifft in der Praxis häufig zentralisierte betriebliche Aktivitäten wie IT Leistungen (Administration von Lizenzen, Betrieb des Helpdesk, Betrieb von Servern, Betreuung des Internet-/Intranetauftritts usw.), Marketing, Einkauf, Finanzen (Unterstützung bei der Budgetplanung, Budgetkontrolle, Kapitalbudgetierung, Entwicklung von Controlling Tools, Unterstützung der Buchhaltung, Behandlung von Forderungen und Verbindlichkeiten), Treasury (sofern nicht anderweitig über konzerninterne Finanzierungstransaktionen vergütet), Rechtsabteilung, Personalwesen usw. Die OECD-RL nennen ebenfalls eine Liste von grundsätzlichen verrechenbaren Dienstleistungen. ${ }^{42}$

Nicht verrechenbar sind jedoch Dienstleistungen, die doppelt erbracht werden, beispielsweise durch eine Abteilung vor Ort sowie auf zentraler Ebene von der Konzernmutter. In diesem Fall würde sich kein zusätzlicher Nutzen für den Leistungsempfänger ergeben. Ob dies der Fall ist, sollte im Einzelfall geprüft werden. Darunter können auch Tätigkeiten fallen, die zur Standardisierung von Abläufen innerhalb eines Konzerns durchgeführt werden. Beispielsweise können mehrere Unternehmen eines Konzerns jeweils eigene Qualitäts- oder Sicherheitsstandards entwickelt haben, die nun auf Konzernebene harmonisiert werden sollen. Fraglich wäre in diesem Zusammenhang nun, ob die Unternehmen für sich genommen jeweils einen Nutzen daraus erzielen, dass diese Standards nun konzernweit vereinheitlicht werden.

\subsubsection{Bestimmung angemessener Verrechnungspreise für Dienstleistungen}

Für die Bestimmung von Verrechnungspreisen für Dienstleistungen kommen in der Regel ein Preisvergleich oder eine kostenbasierte Verrechnung in Betracht (siehe hierzu Ausführungen in Kap. 5.2.4 zur Kostenaufschlagsmethode bzw. Kap. 5.2.5 zur TNMM), wobei letzteres in der Praxis aufgrund der hohen Anforderungen an die Qualität und

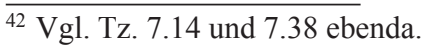


Vergleichbarkeit von Fremddaten eindeutig dominierend ist. Ein Preisvergleich wäre insbesondere dann möglich, wenn die gleiche Leistung unter vergleichbaren Umständen auch an fremde Dritte erbracht wird, etwa Stundensätze für Entwicklungsdienstleistungen oder Entgelte für die Durchführung bestimmter standardisierter Prozesse (beispielsweise Gebühren pro Gehaltsabrechnung, Depotführung, Zugriffe auf bestimmte IT Programme).

Bei der Anwendung der Kostenaufschlagsmethode (oder TNMM) für Dienstleistungen ist - neben der Frage des Gewinnaufschlags - die Ermittlung der Kosten der Dienstleistungen, die an verbundene Unternehmen erbracht werden, von hoher Relevanz (siehe hierzu auch Ausführungen zu Teil vs. Vollkosten in Kap. 5.2.4 zur Kostenaufschlagsmethode). Grundsätzlich wird von der OECD und den meisten Finanzverwaltungen eine direkte Abrechnung von Dienstleistungen im Zuge einer direkten Kostenermittlung bevorzugt. ${ }^{43}$ Dies kann beispielsweise durch das Führen von Zeitaufschrieben, mit denen die (anteilig) geleistete Arbeitszeit pro Dienstleistungsempfänger zugeordnet wird oder durch eine direkte Zuordnung von einzelnen Kosten, die in Bezug auf einen Leistungsempfänger entstanden sind, umgesetzt werden. In der Praxis erweist sich die direkte Methode jedoch häufig als nicht praktikabel: Einerseits werden die Zeitaufschriebe häufig nicht von allen Mitarbeitern konsequent und lückenlos geführt. Andererseits ist es bei vielen Leistungen nicht möglich, diese speziell dem Nutzen einer verbundenen Gesellschaft zuzuordnen, da die Aktivitäten zum Vorteil einer Reihe von Unternehmen durchgeführt werden. Als Beispiel seien hier die Leistungen des zentralen Einkaufs (z. B. Verhandlung von Rahmenverträgen mit strategischen Zulieferern), zentrale Marketingaktivitäten (Messen, Pressearbeit oder Werbekampagnen) oder des Personalwesens (z. B. Entwicklung eines neuen Vergütungssystems oder Ausbildungsprogramms) genannt. Diese Aktivitäten können gleichzeitig zum Nutzen einer Reihe von Unternehmen im Konzern durchgeführt werden, sodass ein Zeitaufschrieb und eine direkte Zuordnung von einzelnen Leistungen mit entsprechenden Kosten nicht möglich ist. In solchen Fällen lassen sich die Kosten verursachungsgerecht am besten anhand von Allokationsschlüsseln auf die von der Dienstleistung profitierenden Unternehmen verteilen (indirekte Methode). Hierbei sollte ein Schlüssel angewendet werden, der zu einer Verteilung der Kosten führt auf die sich auch fremde Dritten geeinigt hätten. Für die Tätigkeiten einer zentralen Einkaufsabteilung könnten die Kosten somit beispielsweise anhand des relativen Einkaufsvolumens mit diesen Zulieferern auf die profitierenden Gesellschaften umgelegt werden. Kosten für Aktivitäten des Personalwesens können beispielsweise anhand der relativen Anzahl der die Tätigkeiten betreffenden Mitarbeiter verteilt werden. Somit sollte im Rahmen der ökonomischen Analyse ebenfalls untersucht werden, welche Allokationsschlüssel im Einzelfall am besten geeignet sind, die Kosten verursachungsgerecht zu verteilen. Die Anwendung eines einheitlichen Umlageschlüssels (wie beispielsweise relative Umsätze) für ein ganzes Bündel von Dienstleistungen empfiehlt sich jedoch im Regelfall nicht, es sollten durchaus zumindest nach Gruppen von Leistungen differenzierte Schlüssel angewendet werden, sofern dies zu einer verursachungsgerechteren Verteilung führt. Die Auswahl der

${ }^{43}$ Vgl. Tz. 7.21 ebenda. 
Allokationsschlüssel sowie eine Begründung der Eignung der Schlüssel sollte im Rahmen der Verrechnungspreisdokumentation diskutiert werden.

Bezüglich eines fremdüblichen Gewinnaufschlags auf die Kosten der Dienstleistung sei auf die Ausführungen in Kap. 5.2.4 zur Kostenaufschlagsmethode bzw. Kap. 5.2.5 zur TNMM verwiesen. Im Regelfall sollte der Gewinnaufschlag anhand empirischer Fremdvergleichsdaten belegt werden. Aus Vereinfachungsgründen gibt es mittlerweile eine Reihe von nationalen und internationalen Regelwerken bzw. Publikationen, die auf allgemein akzeptierte Bandbreiten von Gewinnaufschlagssätzen verweisen. Als Beispiel sei hierzu eine Publikation des EU Joint Transfer Pricing Forum ${ }^{44}$ genannt, nach dem Gewinnaufschläge für sog. Dienstleistungen mit geringer Wertschöpfung in einer Bandbreite von 3 bis $10 \%$ allgemein akzeptiert werden sollten, wobei ein Wert von 5\% in der Praxis am häufigsten angewendet wird. In einigen Ländern, beispielsweise in den USA, gibt es zudem Vorschriften, nach denen bestimmte Leistungen auch ohne Gewinnaufschlag verrechnet werden können. ${ }^{45}$ In der Praxis werden von US Unternehmen diese sog. „White List“" Dienstleistungen daher häufig nur zu Kosten verrechnet.

Neben der Bestimmung von Verrechnungspreisen für Dienstleistungen anhand der Preisvergleichs- oder Kostenaufschlagsmethode können hochwertige, nicht-Routine Dienstleistungen bei fehlenden Fremdvergleichsdaten grundsätzlich auch gemäß ihres Wertbeitrags, der anhand einer detaillierten Wertschöpfungsanalyse zu bestimmen ist, vergütet werden. In der Praxis findet sich dies z. B. bei der Vergütung von hochwertigen und komplexen Einkaufsdienstleistungen im Konzern, die anhand eines Anteils an den Einsparungen durch bessere Lieferantenkonditionen bestimmt wird. Ein solcher Ansatz setzt jedoch voraus, dass der Wertbeitrag und die Einsparungen der zentralen Einkaufsfunktion genau dokumentiert werden können. Sofern die Einsparungen gegenüber Lieferanten nur auf Synergien durch das Bündeln des Einkaufsbedarfs verschiedener Konzerneinheiten zurückzuführen ist, wird mittlerweile die Auffassung vertreten, dass die entsprechenden Vorteile auf die einkaufenden Einheiten gemäß ihres Einkaufsvolumens zu verteilen sind. ${ }^{46}$ Der Einkaufsfunktion an sich sollte nur ein angemessenes Dienstleistungsentgelt (z. B. auf Basis der Kosten der Dienstleistung zuzüglich eines angemessenen Gewinnaufschlags) zugewiesen werden.

\subsubsection{Dienstleistungen mit geringer Wertschöpfung}

In diesem Zusammenhang wird im Folgenden auf die Neuerungen auf OECD Ebene hinsichtlich der Verrechnung von Dienstleistungen aufgrund des BEPS Projekts eingegangen. Das im Jahr 2015 überarbeitete Kap. VII der OECD-RL sieht nun spezielle Regelungen für die Verrechnung sog. Dienstleistungen mit geringer Wertschöpfung („Low value-adding

\footnotetext{
${ }^{44}$ Vgl. Tz. 7.7.2 des EU JTPF Report: Guidelines on low value adding intra-group services, meeting of 4th February 2010.

${ }^{45}$ Vgl. hierzu IRS Regulations zu „Services Cost Method“, § 1.482-9(b) (2).

${ }^{46}$ Vgl. hierzu Tz. 1.162 und Beispiele in Tz. 1.168 und 1.169 OECD (2015), Aligning Transfer Pricing Outcomes with Value Creation, Actions 8-10.
} 
intragroup services") vor, die vor allem zu einer Vereinfachung der Verrechnung für den Steuerpflichtigen führen sollen. Zum anderen ist das Ziel der neuen Richtlinien die Reduzierung der Verlagerung von Gewinnen und Vermögenswerten innerhalb von Konzernen durch eine exzessive Verrechnung sog. „Management Fees“ und „Head Office Expenses“.

Demgemäß können Dienstleistungen, die innerhalb des Konzerns an verbundene Einheiten erbracht werden, als Dienstleistung mit geringer Wertschöpfung angesehen werden, wenn diese

- Lediglich einen unterstützenden Charakter haben,

- Nicht Teil des eigentlichen Kerngeschäftes des Konzerns sind,

- Dabei keine immateriellen Wirtschaftsgüter eingesetzt oder geschaffen werden, und

- Kein signifikantes Risiko mit sich bringen oder schaffen.

Als Beispiele nennt die OECD hier die folgenden Dienstleistungen: Rechnungslegung und Revision, Kreditoren- und Debitorenbuchhaltung, Personal/HR, IT, Überwachung von Gesundheits-, Sicherheits-, Umwelt- und anderer Vorschriften, Öffentlichkeitsarbeit (PR) sowie Rechts- und Steuerdienstleistungen. Als Beispiele für Dienstleistungen, die nicht als „low value adding services"klassifiziert werden können, werden u. a. die folgenden genannt: Dienstleistungen des Kerngeschäftes, Forschungs- und Entwicklungsdienstleistungen, Produktionsdienstleistungen, Vertrieb, Marketing und Verkauf, Finanztransaktionen, Abbau, Erforschung, oder Erarbeitung von Rohstoffen, Versicherungen sowie Dienstleistungen der Geschäftsleitung.

Für die Verrechnung der Dienstleistungen mit geringer Wertschöpfung schlägt die OECD einen vereinfachten Abrechnungsansatz vor, der den Aufwand der Mitwirkungspflichten für Steuerpflichtige reduzieren, die Rechtssicherheit erhöhen und den jeweiligen Finanzverwaltungen eine effizientere Prüfung ermöglichen soll. Die Vorgehensweise wird im Folgenden kurz umrissen: Zunächst sollen die Kosten für die Erbringung der „low value adding services“ auf jährlicher Basis bei allen Konzernmitgliedern ermittelt werden, wobei die Kosten nach Service Kategorien und Kostenstellen getrennt aufgestellt werden sollen. Die Kostenbasis darf keine Kosten für Gesellschafteraktivitäten oder für selbst genutzte Leistungen enthalten. Sofern möglich, sind die Kosten für direkt an einen Leistungsempfänger zuordenbare Dienstleistungen aus dem Kostenpool zu entfernen und direkt abzurechnen. In einem zweiten Schritt werden die ermittelten Kosten über einen oder mehrere Allokationsschlüssel (Kombination) auf die Leistungsempfänger verteilt. Der oder die Allokationsschlüssel sind so auszuwählen, dass diese den Nutzen und die tatsächliche Nachfrage nach der Dienstleistung durch die Empfänger am besten berücksichtigen. Hierzu werden u. a. folgende Beispiele genannt: Personaldienstleistungen (Headcount), IT-Leistungen (Anzahl Nutzer) oder Rechnungslegung (Anzahl relevant Geschäftsvorfälle, Bilanzsumme, Umsatz). Die gewählten Allokationsschlüssel sollen durch den Steuerpflichtigen für die Zuordnung der Kosten der gleichen Dienstleistungskategorie konsistent angewendet werden und - sofern keine wichtigen Gründe für eine Änderung vorliegen - über mehrere Jahre kontinuierlich angewendet werden. Letzteres gilt auch für 
den angewendeten Gewinnaufschlag. Dieser sollte für alle Dienstleistungen mit geringer Wertschöpfung gleich sein und $5 \%$ betragen.

Schließlich ist für den oben beschriebenen vereinfachten Abrechnungsansatz für Dienstleistungen mit geringer Wertschöpfung eine Dokumentation zu erstellen, welche die folgenden Punkte umfassen sollte:

- Beschreibung der unterschiedlichen Kategorien der Dienstleistungen,

- Begründung, wieso die Dienstleistung als eine „low value adding“ Service eingestuft wurde,

- Benennung der Dienstleistungsempfänger,

- Begründung, warum diese Dienstleistungen zentral erbracht werden und eine Beschreibung des (erwarteten) Nutzens für die Empfänger,

- Beschreibung der angewendeten Allokationsschlüssel pro Dienstleistungsart und eine Begründung, warum diese als angemessen angesehen werden,

- Benennung des angewendeten Gewinnaufschlags,

- (Schriftliche) Verträge über die Erbringung der Dienstleistungen und entsprechende Vertragsergänzungen bzw. -änderungen,

- Dokumentation und zu Grunde liegende Berechnungen zur Bestimmung des Kostenpools je nach Kategorie sowie für direkte Einzelabrechnungen, und

- Berechnungen, die die Anwendung des Allokationsschlüssels zur Verteilung der Kostenbasis zeigen.

Hierzu sei noch hinzugefügt, dass die oben aufgezählten Punkte im Allgemeinen auch für sämtliche anderen Dienstleistungen im Konzern dokumentiert werden sollten, um auf eine Betriebsprüfung gut vorbereitet zu sein. In der Praxis werden solche Dokumentationen von vielen Konzerngesellschaften im Rahmen lokaler Betriebsprüfungen für die Verteidigung von Dienstleistungsentgelten benötigt, so dass es sich empfiehlt, eine solche Dokumentation jährlich zentral zu erstellen und den Leistungsempfängern regelmäßig zur Verfügung zu stellen.

\subsubsection{Kostenumlagen oder Cost Contribution Arrangements}

Neben der Verrechnung von Dienstleistungen können sich verbundene Gesellschaften eines multinationalen Konzerns auch dafür entscheiden, gemeinsame Leistungen im Rahmen eines Kostenumlagevertrag („Cost Contribution Arrangement“ oder „CCA“) zu erbringen und auszutauschen. Bei einem Kostenumlagevertrag werden die im Rahmen der Tätigkeit tatsächlich angefallenen Kosten ohne einen Aufschlag weiterbelastet (siehe hierzu einschränkend die nachfolgenden Ausführungen zu dem im Rahmen des BEPS Projekts überarbeiteten Kap. VIII der OECD-RL), während die Kosten bei einem Dienstleistungsvertrag in der Regel mit Gewinnaufschlag weiterbelastet werden. Dem Steuerzahler steht es im Allgemeinen frei einen dieser Ansätze zu wählen, jedoch ist gemäß 
OECD-RL (siehe u. a. Tz. 8.3 ff. des neuen Kap. VIII der OECD-RL) und den deutschen Verrechnungspreisvorschriften ${ }^{47}$ das Vorliegen eines gemeinsamen Interesses des oder der Leistungserbringer sowie des oder der Leistungsempfänger die dringende Voraussetzung für das Vorliegen eines Umlagevertrags. So definieren die VWG-Umlageverträge (Tz. 1.1) einen Umlagevertrag als einen Vertrag, welcher geschlossen wurde, um im gemeinsamen Interesse und in einem längeren Zeitraum durch das Zusammenwirken in einem Pool Leistungen zu erlangen oder zu erbringen. Sofern der Leistungserbringer also nicht selbst von der Leistung profitiert, ist dieser nicht Teils eines Pools und es liegt im Prinzip ein Dienstleistungsvertrag vor. Das bedeutet, dass in diesem Fall eine fremdübliche Vergütung für die Dienstleistung zu verrechnen ist, entweder auf Basis vergleichbarer Marktpreise (sofern vorhanden) oder auf Basis der Kosten zuzüglich eines fremdüblichen Gewinnaufschlags.

Die VWG-Umlageverträge enthalten umfangreiche Dokumentationsanforderungen. Danach bedarf ein Kostenumlagevertrag der Schriftform und soll zumindest die folgenden Elemente enthalten (Tz. 5.1.1):

- Benennung der Poolmitglieder und der sonstigen nahestehenden Nutznießer;

- Genaue Beschreibung der Leistungen, die Vertragsgegenstand sind;

- Ermittlung der umzulegenden Aufwendungen, die Methode der Aufwandserfassung und etwaige Abweichungen;

- Ermittlung des Nutzens, den die jeweiligen Teilnehmer erwarten;

- Ermittlung des Umlageschlüssels;

- Beschreibung der Ermittlung und der Verrechnung des Werts der anfänglichen und späteren Leistungsbeiträge der Poolmitglieder;

- Art und Umfang der Rechnungskontrolle (z. B. bei Vorkasse; Zeitpunkt)

- Bestimmungen über die Anpassung an veränderte Verhältnisse;

- Vertragsdauer;

- Bestimmungen über die Vertragsauflösung sowie ggf. Eintritt oder Austritt von Poolmitgliedern;

- Vereinbarungen über den Zugriff auf die Unterlagen über den Aufwand und die Leistungen des leistungserbringenden Unternehmens;

Im Rahmen des OECD BEPS Projekts wurden die Richtlinien im Jahr 2015 hinsichtlich der Anwendung von Umlageverträgen grundlegend überarbeitet. Ziel war es insbesondere, die Verrechnungspreisregeln für Umlageverträge den tatsächlichen wertschöpfenden Tätigkeiten im Konzern anzupassen. Eine der wichtigsten Neuerungen in diesem Zusammenhang ist, dass künftig die Beiträge der einzelnen Poolmitglieder nicht mehr nur nach den angefallenen Kosten, sondern nach deren Wertbeitrag zu messen sind. In der Praxis

\footnotetext{
${ }^{47}$ Siehe Tz. 1.7 und 2.1 der Grundsätze für die Prüfung der Einkunftsabgrenzung durch Umlageverträge zwischen international verbundenen Unternehmen BMF, Schreiben v. 30.12.1999- IV B 4- S 1341 -14/99, BStB1 1999 I S. 112 („,VWG-Umlageverträge“).
} 
bedeutet dies für die Steuerpflichtigen eine erheblich höhere Komplexität. Während in der Vergangenheit in der Regel die Kosten der Poolmitglieder ermittelt und nach Nutzen aufgeteilt wurden, ist nun für die einzelnen Beiträge der Poolmitglieder ein fremdüblicher Preis zu bestimmen. In der Praxis wird dies häufig über fremdübliche Kostenaufschlagssätze umgesetzt werden, wobei diese je nach Wertbeitrag des Mitglieds unterschieden werden müssten. Eine Ausnahme gilt für sog. „low value adding services“; hier darf auch zukünftig aus Vereinfachungsgründen der Beitrag der einzelnen Mitglieder zum Pool in Kosten gemessen werden. Die neuen und komplexeren Regelungen zielen somit vor allem auf den Austausch von höherwertigen Leistungen im Konzern und insbesondere auf sog. Forschungs- und Entwicklungspools (,F\&E Pools“) ab.

Generell sind F\&E Pools jeweils für Situationen geeignet, in denen mehrere Konzerneinheiten (grenzüberschreitend) gemeinsam immaterielle Wirtschaftsgüter entwickeln und diese im Rahmen ihrer Geschäftstätigkeiten (z. B. Produktion der entwickelten Güter) gemeinsam nutzen. Dadurch können unter Umständen komplexe gegenseitige Lizenzverträge - und ströme vermieden werden. In der Praxis zeigt sich jedoch häufig, dass F\&E Pools in der Umsetzung ebenfalls sehr komplex sein können und haben daher in den letzten Jahren an praktischer Bedeutung verloren. Die Komplexität von F\&E Pools wurde durch die neuen Richtlinien nun weiter erhöht; die wichtigsten Aspekte werden im Folgenden kurz erläutert.

Die grundlegende Voraussetzung für die Anwendbarkeit solcher F\&E Pools ist, dass die Pool-Mitglieder alle einen gemeinsamen Nutzen von den geteilten Aufgaben erwarten, wobei laut OECD der erwartete Nutzen auch in etwa dem eingebrachten Beitrag entsprechenden sollte. Hierzu ist es erforderlich, dass der Inhalt und die Ziele der gemeinsamen F\&E Aktivitäten klar definiert und vertraglich geregelt sind. Jedes Poolmitglied sollte zudem die Absicht haben, die Ergebnisse des F\&E Pools auch selbst zu nutzen, andernfalls sollte die entsprechende Tätigkeit nicht im Rahmen des Pools geteilt werden. Desweiteren hat die OECD erhöhte Substanzanforderungen an ein Poolmitglied definiert. Das bedeutet, dass jedes Pool-Mitglied über entsprechende wirtschaftliche Substanz und Entscheidungsbefugnisse verfügen sollte, um die mit den geteilten F\&E Aktivitäten verbundenen Risiken überwachen und kontrollieren zu können, und falls erforderlich, entsprechende Entscheidungen treffen und Maßnahmen einleiten können. Mit diesen neuen Anforderungen sollen Konstellationen verhindert werden, in denen vor allem niedrig besteuerte Konzerneinheiten mit geringer wirtschaftlicher Substanz über die Mitgliedschaft in einem F\&E Pool möglichst günstig wirtschaftliches (Mit-) Eigentum an wertvollen IWGs erlangen.

Bei der Einrichtung eines F\&E Pools sind die jeweiligen Beiträge der Pool-Mitglieder nach dem Fremdvergleichsgrundsatz zu bewerten. Dies betrifft wertvolles geistiges Eigentum der Mitglieder, das bereits vor der Gründung des Pools entwickelt wurde und von einem Poolmitglied zur Nutzung durch andere Poolmitglieder eingebracht wird. Sofern der zur Gründung des F\&E Pools eingebrachte Wert der IWGs der verschiedenen Mitglieder ungleich ist, sind entsprechende Ausgleichszahlungen auf Basis des erwarteten Nutzens aus den IWGs zu leisten (sog. „Buy-in payments“). Auch während der Laufzeit 
des F\&E Pools sind, wie bereits oben erwähnt, Beiträge nicht anhand ihrer Kosten sondern anhand des fremd- oder marktüblichen Werts anzusetzen. In vielen Fällen wird dies durch Anwendung eines Gewinnaufschlags auf die Kosten erfolgen, jedoch stellen die Kosten nicht unbedingt immer einen angemessen Indikator für den Wertbeitrag von IWGs dar.

Der Wert der geleisteten Beiträge eines Pool-Mitglieds sollte mit dem erwarteten Nutzen des Mitglieds übereinstimmen. Sofern es Abweichungen gibt, sind entsprechende Ausgleichszahlungen zwischen den Mitgliedern zu leisten. Eine große Schwierigkeit besteht jedoch gerade in der Messung des erwarteten Nutzens aus F\&E Pools, da bei F\&E Tätigkeiten in der Regel eine Zeitdifferenz zwischen dem Beitrag zur Entwicklung von wertvollen IWGs und der kommerziellen Nutzung der IWGs besteht. Daher ist häufig zum Zeitpunkt der Entwicklung nicht klar, welches Mitglied später in welchem Umfang von der kommerziellen Nutzung profitieren wird. Praktisch könnte der erwartete Nutzen mit Hilfe von Allokationsschlüsseln gemessen und die entsprechenden tatsächlichen Beiträge zum Pool danach umverteilt werden. Beispielsweise wird in diesem Zusammenhang häufig die relative Produktionskapazität der Mitglieder verwendet, da diese weniger volatil als z. B. relativer Produktumsatz ist. Insbesondere in einem Konzernverbund ist dies jedoch auch kritisch, da die Verschiebung von Produktionskapazität und Standortentscheidungen im Konzern einen erheblichen Einfluss auf die tatsächliche Nutzung der IWGs haben können. Dies bedeutet, dass in der Praxis eine regelmäßige Überprüfung der Verteilung des tatsächlichen Nutzens aus den Pool-Aktivitäten erforderlich ist, was zu entsprechenden Ausgleichszahlungen und Anpassungen der Allokationsschlüssel führen wird.

Sofern Beiträge zu einem F\&E Pool von einem Nicht-Mitglied geleistet werden, sind die Tätigkeiten zunächst mit einem fremdüblichen Preis zu vergüten. Dieser Beitrag sollte dann ebenfalls nach (erwarteten) Nutzen auf die Pool-Mitglieder aufgeteilt werden. Sofern durch den F\&E Pool geschaffene IWGs von Nicht-Mitgliedern genutzt werden, sind entsprechende Lizenzverträge mit den Pool-Mitgliedern abzuschließen. Die Verträge sollten genau spezifizieren, welches Pool-Mitglied welche Anteile an den Lizenzen erhält, um mögliche Quellensteuer Problematiken zu verringern. Diese können sich daraus ergeben, dass der Staat des Lizenznehmers unterschiedliche Regelungen in den jeweiligen DBAs mit den Staaten der Pool-Mitglieder in Bezug auf Besteuerung von Lizenzeinkünften (Quellensteuern) vereinbart hat.

Sofern Konzerneinheiten ihre Mitgliedschaft in einem F\&E Pool beenden oder neue Konzerneinheiten Mitglied des Pools werden, sind in den meisten Fällen entsprechende Ausgleichszahlungen erforderlich (Buy-in oder Buy-out Payments). Hierzu sollten sämtliche geleisteten Beiträge der Mitglieder dem erwarteten Nutzen gegenüberstellt werden. Sofern diese nicht übereinstimmen sind entsprechende Ausgleichszahlungen zu leisten. 


\subsubsection{Verrechnungspreise für immaterielle Wirtschaftsgüter}

\subsubsection{Grundsätzliches}

Die Bestimmung von fremdüblichen Verrechnungspreisen für die Bereitstellung oder Übertragung von IWGs innerhalb von multinationalen Konzern ist von hoher praktischer Bedeutung. Die Gründe dafür sind, dass einzigartige und wertvolle IWGs wie Markennamen oder Technologie heutzutage zu den Hauptwerttreibern in der Wertschöpfungskette von Konzernen gehören. Das heißt mit ihnen ist ein hohes Gewinnpotential verknüpft. Gleichzeitig sind Einkommensströme, die ausschließlich einem bestimmten IWG zuzurechnen sind, nur schwierig direkt zu messen, da diese in der Regel Teil des gesamten Umsatzes mit den Produkten sind, welche mithilfe der IWGs produziert und am Markt vertrieben worden sind. Daher ist eine Bepreisung bzw. Bewertung von IWGs häufig mit Subjektivität und Annahmen verbunden. Diese lassen einen gewissen Spielraum und verstärken die damit verbundene Unsicherheit hinsichtlich der Bewertung. Zudem sind IWGs per Definition sehr mobil und können innerhalb von multinationalen Konzernen in verschiedenen Jurisdiktionen einfach eingesetzt bzw. transferiert werden. Daher sind Verrechnungspreise für IWGs in den letzten Jahren stark in den Fokus von Betriebsprüfungen geraten. Auch die OECD hat im Rahmen des BEPS Projekts verkündet, dass eine falsche Allokation von Gewinnen aus der Nutzung von IWGs in erheblichem Ausmaß zu Gewinnverschiebungen und damit verbundenen Steuerverkürzungen geführt hat. Aus diesem Grund wurde das Kap. VI und Teile der Kap. I und II der OECD-RL, welche sich mit Verrechnungspreisaspekten zu IWGs beschäftigen, grundlegend überarbeitet. Die Überarbeitung fand letztlich im Rahmen des BEPS Projekts statt, obgleich bereits seit 2011 in der OECD über die Notwendigkeit neuer Verrechnungspreisrichtlinien für IWGs beraten wurde und vor dem Start des BEPS Projekts erste Neuentwurfsfassungen für das Kap. VI der OECD-RL in 2012 und 2013 veröffentlicht wurden. Im Folgenden werden die neuen Richtlinien hinsichtlich der Behandlung von IWGs aus Verrechnungspreissicht kurz dargestellt.

Im Rahmen der Verrechnungspreisanalyse im Zusammenhang mit IWGs sind grundsätzlich die folgenden vier Analyseschritte erforderlich: ${ }^{48}$

- Was sind die relevanten IWGs aus Verrechnungspreissicht und welche IWGs sind wichtiger Bestandteil der Wertschöpfungskette im Konzern?

- Welcher Konzerneinheit oder welchen Konzerneinheiten sind die identifizierten IWGs und die entsprechenden Erträge aus den IWGs zuzuordnen?

- Welche Geschäftsvorfälle mit IWGs liegen im Konzern vor?

- Wie können fremdübliche Verrechnungspreise für Transaktionen mit IWGs bestimmt werden?

\footnotetext{
${ }^{48}$ Vgl. hierzu Kap. 6 in OECD (2015), Aligning Transfer Pricing Outcomes with Value Creation,
} Actions 8-10. 
Die OECD definiert IWGs aus Verrechnungspreissicht als ein nicht-materielles oder finanzielles Wirtschaftsgut, welches geeignet ist Gegenstand einer Geschäftsbeziehung zu sein, welches im Eigentum einer (juristischen) Person stehen und von diesem kontrolliert werden kann. ${ }^{49}$ Dabei müssen IWGs aus Verrechnungspreissicht nicht unbedingt auch IWGs für Rechnungslegungszwecke sein. Auch bedeutet ein fehlender rechtlicher Schutz für ein Wirtschaftsgut nicht automatisch, dass es kein IWG aus Verrechnungspreissicht darstellt. Als Beispiele für IWGs ${ }^{50}$ aus Verrechnungspreissicht werden Patente, Knowhow, Geschäftsgeheimnisse, Markenrechte und vertraglich gesicherte Lizenzen genannt. Keine IWGs aus Verrechnungspreissicht sind Gruppensynergien, marktspezifische Charakteristika oder Standortvorteile, da diese nicht durch einen Eigentümer kontrolliert werden können. Letztere können jedoch ebenfalls einen Einfluss auf die Bestimmung von Verrechnungs-preisen haben und sollten separat analysiert werden. Die für die Verrechnungspreisanalyse relevanten IWGs sollten im Rahmen der Funktions- und Risikoanalyse identifiziert und genau benannt werden (siehe hierzu Ausführungen in Kap. 4.4.4).

In einem nächsten Schritt ist auf Basis einer umfassenden Analyse der Funktionen, Risiken und eingesetzten Wirtschaftsgüter der beteiligen Konzerneinheiten zu bestimmen, welchen Konzerneinheiten die Erträge aus den identifizierten IWGs zuzuordnen sind. Als Startpunkt für die Verrechnungspreisanalyse im Zusammenhang mit IWGs sollte zunächst das rechtliche Eigentum an den IWGs und die vertraglichen Beziehungen zwischen den involvierten Konzerneinheiten geprüft und festgestellt werden. ${ }^{51}$ Hierzu können schriftliche Verträge, öffentliche Einträge (z. B. Patent-registrierungen) oder entsprechende Korrespondenz zwischen den beteiligten Parteien herangezogen werden. Allerdings betont die OECD, dass rechtliches Eigentum an IWGs alleine nicht genügt, um einen Anspruch auf eine (vollständige) Entlohnung aus der Bereitstellung der IWGs zu erhalten. ${ }^{52}$ Vielmehr sollen die tatsächlich von allen beteiligten Parteien ausgeübten Funktionen, die im Zusammenhang mit der Entwicklung, der Verbesserung, Aufrechterhaltung, dem Schutz und der Verwertung des IWGs (engl.: ,development, enhancement, maintainance, protection and exploitation“ oder „DEMPE“ Funktionen) stehen, bei der Analyse berücksichtigt werden. Sofern es Abweichungen zwischen dem tatsächlichen Verhalten der Parteien und dem schriftlich fixiertem Vertrag gibt, ist generell das tatsächliche Verhalten der Verrechnungspreisanalyse zu Grunde zu legen. Dies kann bei einer groben Abweichung der vertraglichen Vereinbarung von dem tatsächlichen Verhalten der Parteien zu einer Umklassifizierung von Transaktionen oder Verträgen führen. In diesem Sinne sind die Konzernmitglieder, die wertbeitragende Funktionen in Bezug auf das IWG ausführen, an den durch die Verwertung des IWG antizipierten Gewinnen durch Erhalt einer fremdüblichen Vergütung gemäß ihres relativen Wertbeitrags zu beteiligen. Demzufolge sind dem rechtlichen Eigentümer des IWGs nur dann die Residualgewinne aus der Verwertung

\footnotetext{
${ }^{49}$ Vgl. Tz. 6.6 ff. ebenda.

${ }^{50} \mathrm{Vgl}$. Tz. 6.19 bis 6.29 ebenda.

${ }^{51}$ Vgl. Tz. 6.35 ebenda.

52 Vgl. Tz. 6.42 ebenda.
} 
des IWGs zuzuordnen, wenn dieser die wichtigen Funktionen ausübt und kontrolliert, die dazu benötigten Wirtschaftsgüter inklusive der Kapitalausstattung zur Verfügung stellt sowie die Risiken übernimmt und kontrolliert, die im Zusammenhang mit den wertbeitragenden Funktionen eines IWG stehen.

Die tatsächliche wirtschaftliche Substanz der Transaktion wird somit deutlich stärker gegenüber der vertraglichen Vereinbarung in den Vordergrund gerückt. Damit möchte die OECD vor allem Strukturen bekämpfen, in denen steuergünstig gelegene Konzerneinheiten mit wenig wirtschaftlicher Substanz über eine hohe Eigenkapitalausstattung und entsprechende Verträge der (rechtliche) Eigentümer von wertvollen IWGs werden, und über Lizenzen an Konzerngesellschaften die Residualgewinne aus den wertvollen IWGs erhalten.

Dies bedeutet nicht, dass Forschungs- und Entwicklungsfunktionen im Konzern im Sinne von Auftragsentwicklung nicht an andere Einheiten delegiert oder beauftragt werden können. Vielmehr betont die OECD, dass der Auftraggeber in diesem Fall die tatsächliche Kontrolle über die wertbeitragenden (DEMPE) Funktionen ausüben (können) und über entsprechende Entscheidungskompetenzen verfügen muss. Ob diese theoretischen Anforderungen in der betrieblichen Praxis immer umgesetzt und auf Anfragen von Finanzverwaltungen entsprechend dokumentiert werden können, wird von Industrievertretern noch kritisch gesehen.

Neben der Analyse der wertbeitragenden Funktionen ist auch zu klären, welche Rolle der Bereitstellung von Kapital im Kontext der Schaffung wertvoller IWGs beizumessen ist. Wie bereits erwähnt steht mittlerweile im Vordergrund, welche der beteiligten Konzerneinheiten tatsächlich die wertbeitragenden Funktionen ausüben. Demzufolge ist eine Konzerneinheit, welche nur die Kosten zur Schaffung eines IWG und die mit der Finanzierung einhergehenden Risiken übernimmt, ohne dabei die wichtigen Funktionen und Risiken auszuüben bzw. zu kontrollieren, nicht notwendigerweise zum Erhalt der Residualgewinne aus den IWGs berechtigt. Vielmehr ist eine reine Finanzierungsfunktion durch eine fremdübliche risikoadäquate Verzinsung des eingesetzten Kapitals zu vergüten ${ }^{53}$, wobei die angemessene Bepreisung des Risikos in der Praxis eine komplexe Analyse voraussetzt.

\section{Beispiel 5.7: Finanzierung von Forschungs- und Entwicklungsleistungen ${ }^{54}$}

Die Konzernunternehmen D-GmbH und IP Company vereinbaren im Rahmen eines Auftragsentwicklungsvertrags die Entwicklung neuer technischer IWGs, wobei existierende IWGs der D-GmbH als Grundlage für die Entwicklungsarbeiten dienen werden. Die D-GmbH übernimmt und kontrolliert alle Aktivitäten, die mit der Entwicklung, dem Erhalt und der Verwendung des IWG verbunden sind. Die Entwicklung wird über einen Zeitraum von drei Jahren stattfinden und die gesamten Entwicklungskosten

\footnotetext{
${ }^{53}$ Vgl. Tz. 6.61 ebenda.

${ }^{54}$ In Analogie zu Beispiel 6 des neuen Kapitel VI der OECD-RL.
} 
werden 30 Mio. $€$ betragen. Die IP Company übernimmt die Finanzierung der Entwicklungsleistungen und wird zum rechtlichen Eigentümer des IWG. Nach Beendigung der Entwicklungsarbeiten räumt die IP Company der D-GmbH ein Nutzungsrecht für die entwickelten IWGs ein und die D-GmbH zahlt eine Nutzungslizenz an die IP Company. Mit den neu entwickelten Produkten lassen sich über einen Produktlebenszyklus von 7 Jahren Gewinne von 30 Mio. $€$ pro Jahr erwirtschaften. Davon erhält die IP Company eine Lizenz von der D-GmbH in Höhe von 20 Mio. $€$ pro Jahr. Insgesamt erzielt der Konzern einen Gewinn von 180 Mio. $€$ aus den IWGs (30 Mio. € Gewinn über 7 Jahre abzüglich 30 Mio. $€$ Entwicklungskosten), davon erhält die IP Company 110 Mio. $€$ und die D-GmbH 70 Mio. $€$.

Im Rahmen einer Betriebsprüfung bei der D-GmbH wird diese Struktur genau geprüft und eine umfangreiche Funktions- und Wertschöpfungsbeitragsanalyse durch die Finanzverwaltung durchgeführt. Aufgrund der fehlenden wirtschaftlichen Substanz und Kompetenzen bei der IP Company, die wertbeitragenden (DEMPE) Funktionen zu steuern und zu kontrollieren, wird der Beitrag der IP Company zur Entwicklung des IWG als reine Finanzierungsfunktion der Forschungs- und Entwicklungsleistungen klassifiziert. Nach Prüfung der realistischen Handlungsalternativen der D-GmbH und IP Company geht die Betriebsprüfung davon aus, dass der IP Company lediglich ein Finanzierungsgewinn in Form einer risikoangepassten Verzinsung des eingesetzten Kapitals von $10 \%$ zusteht. Daraus ergibt sich, dass für die IP Company lediglich ein Gesamtgewinn von 16,67 Mio. € für ihre Finanzierungsfunktion angemessen wäre, woraus in diesem (vereinfachten) Beispiel eine Einkommensanpassung zu Gunsten der D-GmbH i. H. v. 93,3 Mio. € resultieren würde.

Das obige Beispiel verdeutlicht, wie Finanzverwaltungen - den neuen Ausführungen der OECD folgend - künftig entsprechende Strukturen prüfen werden. Dies bedeutet nicht, dass aus Verrechnungspreissicht grundsätzlich keine Auslagerung von Entwicklungstätigkeiten an andere Konzerngesellschaften im Wege von Auftragsentwicklungsverträgen mehr möglich ist (vgl. hierzu etwa Beispiele 14 und 15 des neuen Kap. VI der OECD-RL). Jedoch sind die Substanzanforderungen an die auslagernde Gesellschaft deutlich verschärft worden und im Fokus steht, welche Gesellschaft tatsächlich und in welchem Umfang an den wertbeitragenden Funktionen zur Schaffung wertvoller IWGs beteiligt war.

Für die Verrechnungspreispraxis bedeutet dies, dass es nicht mehr genügt lediglich zu prüfen, welche Partei die Aufwendungen für Forschung und Entwicklung finanziert und vertraglich die damit verbundenen Risiken getragen hat. Vielmehr ist nun der Wertbeitrag aller beteiligten Parteien, auch vor dem Hintergrund der jeweiligen realistischen Handlungsalternativen zu analysieren. Auch ist darauf abzustellen, ob die Partei, welche die Risiken vertraglich übernommen hat, überhaupt in der Lage war, die Risiken zu kontrollieren und im Falle des Risikoeintritts überhaupt hätte tragen können. Schließlich bedeutet dies, dass gemäß den neuen Anforderungen der OECD die Zuordnung der Erträge aus IWGs in der Praxis deutlich komplexer und dadurch auch mit mehr Unsicherheit verbunden sein wird. 
Ein weiterer Schritt im Rahmen der Verrechnungspreisanalyse ist die Identifikation von Geschäftsvorfällen im Konzern im Zusammenhang mit IWGs. Grundsätzlich gibt es Transaktionen, in denen IWGs übertragen oder die Rechte zur Nutzung von IWGs überlassen werden. Typischerweise handelt es sich dabei um die Übertragung des Eigentums an IWGs im Konzern, die Lizenzierung der Nutzungsrechte oder die gemeinsame Nutzung von IWGs im Rahmen von F\&E Pools (siehe hierzu Ausführungen in Abschn. 5.4.2)

Zum anderen gibt es Transaktionen, in denen IWGs im Rahmen von Warenlieferungen oder Dienstleistungen eingesetzt werden. Bei letzteren wird in der Praxis häufig eine Betrachtung des Gesamtgeschäfts und weniger eine isolierte Analyse des Wertbeitrags des involvierten IWGs vorgenommen (beispielsweise wird bei eine Warentransaktion nicht jeweils ein Verrechnungspreis für das IWG, die Produktionsfunktion und das verarbeitete Material ermittelt). Somit sei auf die generellen Ausführungen in diesem Kapitel zu Verrechnungspreismethoden und ihre jeweilige Anwendbarkeit verwiesen. Nichtsdestotrotz sollten die in solchen Transaktionen eingesetzten IWGs im Rahmen der Verrechnungspreis- und Vergleichbarkeitsanalyse identifiziert und benannt werden.

\subsubsection{Verrechnungspreismethoden im Kontext immaterieller Wirtschaftsgüter}

Hinsichtlich der Ermittlung von fremdüblichen Verrechnungspreisen für die Lizenzierung oder Übertragung von IWGs im Konzern finden grundsätzlich die in diesem Kapitel vorgestellten OECD Verrechnungspreismethoden Anwendung. Da es in der Praxis aufgrund des oftmals einzigartigen Charakters von IWGs häufig keine zuverlässigen Fremdvergleichsdaten gibt, sind jedoch auch andere Methoden (insb. Profit Split sowie Bewertungsmethoden) angemessen.

Als wichtige Vergleichbarkeitsfaktoren für die Verrechnungspreisanalyse im Zusammenhang mit IWGs nennt die OECD eine Reihe von Merkmalen, die im Rahmen der Analyse von Bedeutung sein können, u. a.. ${ }^{55}$

- Exklusivität der Nutzungsrechte,

- Länge und Umfang des rechtlichen Schutzes,

- Geografische Nutzungsrechte,

- Wirtschaftliche Nutzungsdauer,

- Grad der Entwicklung des IWGs,

- Weiterentwicklungs- und Verbesserungsrechte,

- Erwarteter zukünftiger Nutzen

Insbesondere im Zusammenhang mit IWGs betont die OECD Ihre Präferenz für zweiseitige Verrechnungspreismethoden, d. h. die Fremdüblichkeit des Verrechnungspreises sollte vor dem Hintergrund der ausgeübten Funktionen, übernommenen Risiken, eingesetzten Wirtschaftsgütern und realistischen Handlungsalternativen der involvierten

${ }^{55}$ Vgl. Tz. 6.197 bis 6.127 ebenda. 
verbundenen Einheiten beurteilt werden. Einseitige Ansätze wie die Wiederverkaufspreismethode oder die TNMM werden als nicht zuverlässige Methoden für die Verrechnungspreisfindung angesehen. ${ }^{56}$ In der Praxis werden fremdübliche Lizenzgebühren zum Teil so bestimmt, dass dem Lizenznehmer nach Bezahlung der Lizenzgebühr noch ein angemessener Restgewinn im Einklang mit der Verteilung der Funktionen, Risiken und Wirtschaftsgütern zwischen den beiden verbundenen Geschäftspartnern verbleibt. Einen solchen Ansatz bezeichnet man als „Residual-TNMM“ oder „Absauglizenz“, da der angemessene Routinegewinn für den Lizenznehmer anhand der TNMM bestimmt wird. Dieser Ansatz ist jedoch umstritten, da nicht in allen Fällen geschlossen werden kann, dass der gesamte über die Routinevergütung hinausgehende Gewinn dem lizensierten IWG zuzuordnen ist. Daher ist ein solcher Ansatz aus Sicht der OECD akzeptabel, wenn nach umfangreicher Funktions- und Risikoanalyse festgestellt wird, dass der Lizenzgeber sämtliche wichtigen IWGs in die Wertschöpfungskette einbringt und der Lizenznehmer nur relativ einfache Funktionen und Risiken ausführt.

Die Anwendung von kostenbasierten Ansätzen zur Bewertung von IWG führt generell nicht zu zuverlässigen und fremdüblichen Ergebnissen, da nur ganz selten ein $\mathrm{Zu}-$ sammenhang zwischen den Kosten zur Entwicklung und dem Wert eines IWG besteht. ${ }^{57}$ Dies gilt auch für noch erst teilweise entwickelte IWGs. In Ausnahmefällen kann eine kostenbasierte Bewertung des IWGs zuverlässig sein, beispielweise wenn es sich um nicht einzigartige und nicht besonders wertvolle IWGs handelt, die überwiegend konzernintern verwendet (z. B. intern genutzte Software) und nicht am Markt gegenüber Kunden angeboten werden.

In der Vergangenheit wurden zur Bestimmung fremdüblicher Lizenzsätze oder zur Bewertung von IWGs aufgrund ihrer Einfachheit häufig Daumenregeln verwendet. Die in Deutschland bekannteste ihrer Art ist die „Knoppe-Formel“" (im englischen Sprachraum meist als ,25\%-Rule“ oder „Goldscheider-Rule“ bekannt). Nach der Knoppe-Formel ist der Lizenzsatz für ein IWG so zu bestimmen, dass dem Lizenzgeber zwischen 25 und $33 \%$ des Gewinns aus der Vermarktung des IWGs verbleiben. Die Verwendung solcher Daumenregeln ist in der Praxis sehr umstritten, da es keine ökonomisch oder empirisch begründbare Evidenz für diese Regeln gibt. Auch ist nicht klar, warum diese Regeln für alle Industrien gelten sollen. Daher ist von der Verwendung dieser Daumenregeln eher abzuraten. Auch gemäß OECD können Daumenregeln nicht zur Bewertung von IWGs herangezogen werden. ${ }^{58}$

Die in der Praxis am häufigsten eingesetzten Verrechnungspreismethoden in Bezug auf IWGs sind die Preisvergleichsmethode oder Gewinnaufteilungsmethode. Auch die OECD sieht diese Methoden als die grundsätzlich am besten geeigneten Methoden an. ${ }^{59}$ Hinsichtlich der Preisvergleichsmethode sei jedoch an die hohen Anforderungen an die

\footnotetext{
${ }^{56}$ Vgl. Tz. 6.141 ebenda.

${ }^{57}$ Vgl. Tz. 6.142 ff. ebenda.

${ }^{58}$ Vgl. Tz. 6.144 ebenda.

${ }^{59} \mathrm{Vgl}$. Tz. 6.145 ebenda.
} 
Vergleichbarkeit einer konzerninternen Transaktion mit Transaktionen zwischen fremden Dritten erinnert (siehe Ausführung in Abschn. 5.2.2 oben.). Da Informationen über Lizenzsätze zwischen fremden Dritten grundsätzlich in öffentlich zugänglichen Datenbanken gefunden werden können, findet diese Methode bei vielen Steuerpflichtigen Anwendung. Allerdings sind die identifizierten Vergleichsverträge einer umfassenden Prüfung der relevanten Vergleichbarkeitskriterien zu unterziehen, um zu bestimmen, ob es sich zumindest um eingeschränkt vergleichbare Fremdvergleichsdaten handelt. Selbst wenn ausreichend zumindest eingeschränkt vergleichbare Lizenzverträge gefunden werden können, ist zu empfehlen, die Ergebnisse und die entsprechende Gewinnverteilung einer Plausibiltätsprüfung durch Anwendung einer zweiten, gewinnorientierten Methode zu unterziehen.

Bezüglich der Anwendung der Gewinnaufteilungsmethode sei auf Abschn. 5.2.1 in diesem Kapitel verwiesen. Diese Methode könnte u. a. zur Ermittlung einer fremdüblichen Lizenz für die Nutzung von IWGs verwendet werden, wenn die Preisvergleichsmethode aufgrund nicht vorhandener Vergleichsdaten nicht anwendbar ist. Für diese Methode ist es von großer Wichtigkeit, dass die gemeinsamen Gewinne aus der Nutzung der IWGs der beteiligten Parteien, die entsprechenden Kosten und der jeweilige Wertbeitrag der Parteien zuverlässig ermittelt werden können. Die Bestimmung des jeweiligen Wertbeitrags hat dabei auf einer detaillierten Analyse der Funktionen, Risiken und eingesetzten Wirtschaftsgüter der beteiligten Parteien zu basieren. Wie bereits in Abschn. 5.2.1 erwähnt, wird eine genauere Anleitung hinsichtlich der Anwendung der Gewinnaufteilungsmethode derzeit von der OECD erarbeitet.

Sofern die Preisvergleichsmethode für die Übertragung eines IWGs im Konzern nicht anwendbar ist, sind grundsätzlich Bewertungsmethoden für die Bestimmung eines fremdüblichen Verrechnungspreises denkbar. Hier wird insbesondere auf Ertragswertverfahren (Discounted Cash Flow Methode) verwiesen, deren Anwendung stets nach dem Fremdvergleichsprinzip zu erfolgen hat. Die OECD geht hier nicht direkt auf einzelne Bewertungsmethoden ein, so dass grundsätzlich verschiedenen betriebswirtschaftliche bewertungsverfahren Anwendung finden können. Die Bewertung von IWGs hängt sehr stark von der Qualität und Zuverlässigkeit der verwendeten Finanzdaten, Annahmen und Parameter ab. Insofern ist es von höchster Bedeutung, diese mit größtmöglicher Sorgfalt herzuleiten, für spätere Betriebsprüfungen zu dokumentieren und zu begründen. Insbesondere sorgfältig zu evaluieren und dokumentieren sind: i) die prognostizierten Finanzdaten, ii) angenommene Wachstumsraten für Umsätze, Kosten usw., iii) die Diskontrate, iv) die wirtschaftliche Nutzungsdauer des IWGs und der Endwert (Terminal Value), v) Steuern, und vi) die Form der Vergütung. ${ }^{60}$ Da die Ergebnisse der Bewertung sehr stark von subjektiven Annahmen abhängig sein können, sind grundsätzlich Sensitivitätsanalysen hinsichtlich der relevanten Parameter zu empfehlen.

Eine erhebliche praktische Schwierigkeit bei der Bewertung von IWGs besteht darin, die Finanzdaten (d. h. Cash Flows), die dem zu bewertenden IWG zugerechnet werden können, zuverlässig zu prognostizieren. In der Praxis greifen Bewertungsmethoden zur

${ }^{60}$ Vgl. Tz. 6.163 bis 6.180 ebenda. 
Herleitung dieser IWG spezifischen Cash-flows) häufig auf Informationen über marktübliche Lizenzsätze aus Datenbanken mit Lizenzverträgen zurück (z. B. Relief-from-Royalty Methode). Da hierbei jedoch das gleiche Datenmaterial wie bei der Preisvergleichsmethode verwendet wird, führen diese Bewertungsmethoden per se nicht notwendigerweise zu einer Verbesserung hinsichtlich der Vergleichbarkeit. Alternativ könnten die IWG spezifischen Cash Flows mittels einer gewinnorientierten Lizenz bzw. einer Residualgewinnanalyse, wie oben beschrieben, ermittelt werden. D. h. auch bei einem solchen Ansatz würde man zunächst auf die üblichen Verrechnungspreisansätze zurückgreifen, um die der Bewertung zu Grunde liegenden Daten zu bestimmen. Eine deutliche Verbesserung der Datenqualität für eine Bewertung von IWGs kann anhand ausgefeilter statistischer Methoden, z. B. Conjoint Analysen, erzielt werden. Bei diesen Ansätzen wird über eine Befragung von unverbundenen Marktteilnehmern (d. h. Kunden oder potentiellen Kunden) die Mehrzahlungsbereitschaft für ein bestimmtes Gut oder Produktmerkmal untersucht. Das entsprechende Ergebnis (z. B. Preisaufschläge) kann dann in die Bewertung einfließen.

Aufgrund der Unsicherheit hinsichtlich der Bewertung von IWGs für Verrechnungspreiszwecke wurde von der OECD im Rahmen des BEPS Projekts weitere Richtlinien entwickelt, wie mit sogenannten „Hard-to-value-intangibles“6 („HTVI“6) umzugehen ist. ${ }^{61}$ Grundsätzlich sollen diese Richtlinien der jeweiligen prüfenden Finanzverwaltung erlauben, die Angemessenheit der Verrechnungspreise für HTVIs, welche auf Basis von ex-ante Finanzdaten bestimmt wurden, anhand von ex-post Finanzergebnissen zu überprüfen. Damit wird im Prinzip dem Steuerpflichtigen zusätzliche Last aufgebürdet, die einer Bewertung zu Grunde liegenden Daten sehr genau zu ermitteln und zu dokumentieren. Gemäß OECD Bericht liegt ein HTVI vor, wenn zum Zeitpunkt der Übertragung im Konzern keine zuverlässigen Fremdvergleichswerte für das IWG vorliegen und die erwarteten Finanzergebnisse aus der Nutzung des IWGs und weitere Bewertungsannahmen nur mit hoher Unsicherheit bestimmt werden können. ${ }^{62}$ Dementsprechend liegen Transaktionen mit HTVI vor, wenn ${ }^{63}$

- das IWG zum Zeitpunkt der Übertragung im Konzern nur teilweise entwickelt ist,

- die kommerzielle Nutzung des IWGs voraussichtlich erst einige Jahre nach der Übertragung beginnen wird,

- das IWG an sich nicht unter die Definition von HTVI fällt, jedoch maßgeblich zur Entwicklung von HTVIs beiträgt,

- die kommerzielle Nutzung des IWGs zum Zeitpunkt des Transfers neuartig ist und Finanzdaten daher aufgrund fehlender Vergleichswerte nur schwer prognostizieren sind,

- ein IWG im Sinne der Definition von HTVI für eine Einmalzahlung übertragen wurde,

- das IWG im Rahmen eines Cost Contribution Arrangements entwickelt wurde oder genutzt werden soll.

\footnotetext{
${ }^{61}$ Vgl. Tz. 6.186 ff. ebenda.

${ }^{62} \mathrm{Vgl}$. Tz. 6.189 ebenda.

${ }^{63}$ Vgl. Tz. 6.190 ebenda.
} 
$\mathrm{Da}$ in den oben beschriebenen Szenarien große Informationsasymmetrien zwischen Steuerpflichtigen und der prüfenden Finanzverwaltung bestehen, ist es für die jeweilige Finanzverwaltung schwierig die Zuverlässigkeit der Annahmen zu überprüfen. Entsprechend soll es daher der Finanzverwaltung im Rahmen von Betriebsprüfungen ermöglicht werden, die Zuverlässigkeit der „ex-ante“ Informationen anhand von „ex-post“" Daten zu überprüfen und darauf basierend ggf. Verrechnungspreisanpassungen vornehmen. Sofern eine der folgenden Annahmen erfüllt ist, sollen jedoch keine Anpassungen auf Basis von „ex-post“" Informationen vorgenommen werden:

- der Steuerzahler macht umfassende Detailangaben darüber, wie die prognostizierten Finanzdaten und Parameter (z. B. Risikoaufschläge) ermittelt wurden. Darüber hinaus erbringt der Steuerzahler ausreichend Nachweise darüber, dass signifikante Unterschiede zwischen den prognostizierten und den tatsächlichen Finanzzahlen aufgrund von unvorhersehbaren und außergewöhnlichen Entwicklungen zustande kamen, oder auf die Eintrittswahrscheinlichkeit von vorhersehbaren Entwicklungen zurück zu führen sind, welche zum Zeitpunkt der Transaktion nicht deutlich über- oder unterschätzt wurden;

- die Übertragung des HTVI durch ein bi- oder multilaterales APA zwischen den Ländern des Erwerbers und des Veräußerers für den infrage stehenden Zeitraum abgedeckt ist;

- die deutlichen Abweichungen zwischen den prognostizierten und den tatsächlichen Finanzzahlen führen nicht zu einer Verminderung oder Erhöhung des vereinbarten Übertragungswerts für das HTVI von mehr als $20 \%$;

- innerhalb von fünf Jahren der Kommerzialisierung des IWGs durch den Erwerber nach der Übertragung kam es nicht zu Abweichungen zwischen den prognostizierten und den tatsächlichen Finanzzahlen von mehr als $20 \%$.

Die Ausführungen der OECD in Bezug auf die HTVIs verdeutlichen, dass Bewertungen von IWGs für die Übertragung im Konzern künftig einer noch genaueren Prüfung durch die Finanzverwaltungen unterzogen werden. Dies bedeutet, dass die Bestimmung der zu Grunde liegenden Finanzdaten und Annahmen weiter an Bedeutung gewinnen wird. Insbesondere sollten alle wichtigen Parameter möglichst genau substantiiert und dokumentiert werden. Zudem ist zu empfehlen, Sensitivitätsanalysen in Bezug auf den Einfluss der verschiedenen Bewertungsparameter (z. B. Wachstumsraten, Laufzeiten, Diskontsätze) auf den Übertragungswert durchzuführen. Je sensitiver die Bewertungsergebnisse auf die Variation eines Parameters reagieren, umso genauer ist dieser Parameter mit einer Analyse zu untermauern und entsprechend für eine spätere Vorlage im Rahmen einer Betriebsprüfung zu dokumentieren. 


\subsubsection{Finanzierungstransaktionen}

\subsubsection{Allgemeines}

Konzerninterne Finanzierungstransaktionen haben in den letzten Jahren ein stetig wachsendes Interesse als Mittel der Steuerplanung von Konzernen erfahren. Gleichsam sind diese Transaktionen auch in den Fokus der Prüfung durch die Finanzverwaltung in vielen Ländern gerückt. Die deutsche Finanzverwaltung nimmt zu konzerninternen Finanztransaktionen bereits in Abschn. 4 der VWG 1983 Stellung. Zudem existieren verschiedene Entscheidungen des Bundesfinanzhofs („BFH“), welche bei der Beurteilung der Angemessenheit des Zinssatzes im Rahmen von konzerninternen Darlehensvergaben zu berücksichtigen sein könnten.

Im Fokus stehen dabei insbesondere Transaktionen wie konzerninterne Darlehen, Cash Pooling, Vergabe von Garantien, Factoring usw. Neben der Höhe der Verrechnungspreise für konzerninterne Finanztransaktionen sind bei der ökonomischen Analyse u. a. folgende Fragestellungen relevant:

- nationale Regelungen für die steuerliche Abzugsfähigkeit von Zinsen auf Fremdkapital, welches von verbundenen Unternehmen aufgenommen wurde (vergleiche beispielsweise die deutschen Regelungen zur Zinsschranke in § 4h EStG),

- die wirtschaftliche Substanz des Darlehensgebers oder Cash Pool Masters (insbesondere bei konzerninternen Finanzierungsgesellschaften in Niedrigsteuerländern), sowie

- das Standhalten des Nachweises, dass die Transaktion konsistent mit dem Fremdvergleichsgrundsatz ist bzw. dass die Konditionen (z. B. Kündigungsoptionen) als solche fremdüblich sind.

So ist jeweils vor Abschluss einer konzerninternen Finanzierungstransaktion zu prüfen, ob das Unternehmen die Transaktion unter Beachtung der bestehenden Finanztransaktionen mit ihren spezifischen Laufzeiten und Kündigungsoptionen auch eingehen würde, wenn die Parteien unverbunden wären. Unverbundene Parteien würden vor Aufnahme von Fremdkapital prüfen, ob die erforderlichen liquiden Mittel ggf. aus anderen günstigeren Quellen unter Beachtung von Laufzeitunterschieden beschafft werden können. Beispielsweise könnten folgende Konstellationen kritisch und nicht mit dem Fremdvergleichsgrundsatz konform sein:

- Aufnahme von Fremdkapital von verbundenen Unternehmen, während gleichzeitig Forderungen aus Darlehen gegenüber anderen Unternehmen bestehen, die niedriger verzinst werden und gekündigt werden könnten,

- Beibehaltung eines jederzeit kurzfristig kündbaren Darlehens mit fixen Zinssätzen, obwohl die zur Zeit vorherrschenden Kreditzinsen auf dem Markt deutlich günstiger sind,

- Hohe Bestände im konzerninternen Cash Pool über einen langen Zeitraum, sowie ggf. gleichzeitig Aufnahme von Fremdkapital. 


\subsubsection{Darlehenstransaktionen}

Hinsichtlich der Bestimmung eines angemessen Zinssatzes für konzerninterne Darlehenstransaktionen ist wiederum der Fremdvergleichsgrundsatz maßgeblich. Auch die deutsche Finanzverwaltung ist der Auffassung, dass bei der Vergabe von Fremdkapital an ein verbundenes Unternehmen derjenige Zinssatz den Verrechnungspreis darstellt, der zwischen unverbundenen Unternehmen vereinbart worden wäre. ${ }^{64}$ Bei der Bestimmung des Zinssatzes müssen daher alle Umstände des Einzelfalls berücksichtigt werden. Die Prüfung der Finanzverwaltung basiert auf folgenden Kriterien. ${ }^{65}$

- Sicherheiten und Kreditwürdigkeit des Kreditnehmers,

- Währung und damit zusammenhängende Risiken,

- Zweck der Kreditaufnahme,

- Kredithöhe,

- Berücksichtigung von Kosten,

- Andere Kriterien, wie die Berücksichtigung der aktuellen Lage an Finanzmärkten.

Wie in Abschn. 5.2.2.2 bereits erläutert, kann bei konzerninternen Finanztransaktionen aufgrund der guten Verfügbarkeit von Kapitalmarktdaten häufig ein Preisvergleich durchgeführt werden. Eine generell mögliche Vorgehensweise bei der ökonomischen Analyse von konzerninternen Darlehen wurde in Abschn. 5.2.2.2 bereits anhand eines Beispiels erläutert. Als Ergänzung sei angemerkt, dass bei sogenannten Durchleitkrediten (d. h. der Darlehensgeber hat das Kapital für das konzerninterne Darlehen vorher bei einem fremden Dritten aufgenommen) die Refinanzierungskosten des Darlehensgebers für die konzerninternen Konditionen beachtet werden sollten. So haben die deutsche Finanzverwaltung und der BFH festgestellt, dass in einem solchen Fall lediglich ein Aufschlag (Handling Margin oder Spread) auf die externen Refinanzierungskosten durch den Darlehensgeber zu veranschlagen ist. In einem Urteil von 1990 entschied der BFH, dass die Höhe des Zinssatzes zwischen verbundenen Unternehmen in engem Zusammenhang mit den Refinanzierungskosten des Kreditgebers gesehen werden soll. ${ }^{66}$ In dieser Entscheidung wurde betont, dass sich, falls der Kreditgeber die konzerninternen Finanzierungstransaktion durch die Aufnahme von Fremdkapital finanziert hat, der Zinssatz des Kredites an das verbundene Unternehmen nach dem Fremdkapitalzinssatz zu richten hat, zuzüglich eines fremdüblichen Gewinnaufschlages. Dies könnte jedoch dazu führen, dass der Darlehensnehmer deutlich bessere Konditionen als auf „Stand Alone“ Basis erhält, da die Finanzierungsgesellschaft unter Umständen eine bessere Kreditwürdigkeit als der konzerninterne Darlehensnehmer hat.

\footnotetext{
${ }_{64} \mathrm{Vgl} . \mathrm{Tz} 4.2 .1 \mathrm{VWG} 1983$.

${ }^{65} \mathrm{Vgl}$. Tz 4.2.2 VWG 1983.

${ }^{66}$ BFH-Urteil vom 28 Februar 1990, BStB1. 1990 II, S. 649.
} 


\subsubsection{Cash Pooling}

Bei der ökonomischen Analyse von konzerninternen Cash Pools ist die Verteilung der Funktionen und Risiken zwischen Cash Pool Master und den Cash Pool Teilnehmern maßgeblich für die Bestimmung von fremdüblichen Konditionen für Soll- bzw. Habenpositionen der Teilnehmer. Im Allgemeinen ist ein Cash Pool eine zentrale Einrichtung einer Gruppe von Unternehmen, um einen kurzfristigen Überschuss oder Bedarf an liquiden Mitteln unter den Teilnehmern auszugleichen. Die Teilnehmer mit Überschüssen werden diese in den Cash Pool einlegen und dafür einen positiven Zinssatz erhalten. Gleichsam werden die Teilnehmer mit kurzfristigem Finanzierungsbedarf diese Liquidität nutzen und für das geliehene Geld einen entsprechenden Zins bezahlen. Die Gesellschaft, die den Cash Pool verwaltet und führt, wird als Cash Pool Master bezeichnet. Dieser ist in der Regel für die zentrale Verhandlung der Zinskonditionen des Pools mit Banken zuständig. Darüber hinaus legt der Cash Pool Master die überschüssige Liquidität des Pools, sofern vorhanden, bei Banken ein bzw. beschafft kurzfristigen Finanzierungsbedarf bei den Banken, falls die Einlagen in den Cash Pool nicht ausreichen. Durch die Einrichtung des Pools ist es nicht erforderlich, dass alle Teilnehmer diese Geschäfte mit Banken abwickeln müssen, da dies durch den Cash Pool Master geschieht. Aus diesem Grund ergeben sich für die Cash Pool Mitglieder Vorteile wie Skalenerträge (d. h. bessere Konditionen mit Banken durch zentrale Verhandlung des Cash Pool Masters) und Handelsgewinne durch das interne Ausgleichen von Soll- und Habenpositionen der Teilnehmer des Pools, ohne eine Bank dazwischen zu schalten. Allerdings entstehen durch die Einrichtung eines Cash Pools auch Verwaltungskosten bzw. bestehen Risiken wie Währungs- und Kreditausfallrisiken.

Um fremdübliche Konditionen für die Haben- und Soll Positionen eines Cash Pools zu bestimmen bzw. die Fremdüblichkeit der Transaktionen eines Cash Pools zu dokumentieren, müssen in einem ersten Schritt die vom Cash Pool Master und den Teilnehmern wahrgenommenen Funktionen sowie getragenen Risiken ermittelt werden. Auch die vertraglichen Bedingungen zwischen den beiden Parteien sowie fremden Dritten müssen bestimmt werden. Basierend auf dem Funktions- und Risikoprofil des Cash Pool Masters wird dann eine geeignete Verrechnungspreismethode für den Cash Pool gewählt. Beispielsweise kann es sich dabei um die Kostenaufschlagsmethode handeln, falls der Cash Pool Master reine Routinetätigkeiten ausübt und keine signifikanten Risiken trägt. In der Regel werden dann die Zinssätze für Haben- und Sollpositionen im Cash Pool so bestimmt, dass der Cash Pool Master einen Spread auf die Haben- und Sollkonditionen mit der Bank erhält, der es ihm unter Beachtung der üblichen Cash Pool Bewegungen ermöglicht, seine administrativen Kosten zu decken und einen angemessenen Routinegewinn zu erwirtschaften.

Andererseits kann die Gewinnaufteilungsmethode zum Tragen kommen, falls der Cash Pool Master keine Routinetätigkeiten ausführt und gewisse Risiken im Zusammenhang mit den Cash Pool Transaktionen trägt. Hierzu wäre es zunächst erforderlich, den (erwarteten) ökonomischen Gewinn des Cash Pools zu ermitteln. Dieser ermittelt sich im Prinzip aus zwei Hauptquellen: Zum einen kann der Cash Pool Master durch seine bessere Verhandlungsmacht vorteilhaftere Zinskonditionen mit Banken vereinbaren, als dies auf alleiniger Basis aller Teilnehmer der Fall wäre. Zum anderen können Handelsgewinne aus 
dem Ausgleich der Soll und Haben Bilanzen der Cash Pool Mitglieder realisiert werden, die sonst von Banken realisiert werden würden. Der ökonomische Gewinn der einzelnen Mitglieder des Cash Pools wird dann durch den Vergleich der Cash Pool Konditionen mit Marktzinssätzen und den Cash Bewegungen im Pool ermittelt. Da der für einen gruppenweiten Cash Pool gewählte Zinssatz maßgeblich für die Allokation der ökonomischen Gewinne zwischen dem Cash Pool Master und den Mitgliedern des Cash Pools ist, sollte der (erwartete) ökonomische Gewinn der jeweiligen Gesellschaft ermittelt werden. Wurde der (erwartete) ökonomische Gewinn durch den Cash Pool geschätzt, wird anschließend der (erwartete) ökonomische Gewinn nach relativem Beitrag des Cash Pool Master und der Mitglieder im Cash Pool aufgeteilt. Dies könnte beispielsweise so ausgestaltet sein, dass der Gewinnanteil des Cash Pool Masters als der relative Gewinn aus vorteilhaften Konditionen am Gesamtgewinn des Cash Pools bestimmt wird, da der Beitrag des Cash Pool Masters vor allem im Aushandeln von vorteilhaften Konditionen mit Banken besteht. Der übrige Gewinn könnte auf die einzelnen Cash Pool Mitglieder je nach Beitrag zum Cash Pool (welcher in Form von Einlagen bzw. Ausleihungen erbracht wird) zugewiesen werden. Basierend auf der Allokation der (erwarteten) ökonomischen Gewinne, können Zinssätze bzw. Zinsmargen kalkuliert werden, welche diese Allokation unterstützen.

Schließlich könnte auch die Preisvergleichsmethode zur Analyse von Cash Pool Konditionen Anwendung finden. Dabei würden sich die Konditionen des Cash Pools anhand von marktüblichen Zinssätzen (beispielsweise für kurzfristige Übernacht-Einlagen oder Überziehungskredite) bestimmen. In einem solchen Fall würde jedoch der gesamte Cash Pool Gewinn dem Cash Pool Master zufallen, was im Falle einer Prüfung durch die Finanzverwaltung zu Diskussionen führen kann.

Hinsichtlich der Auswahl der angemessenen Verrechnungspreismethode vertritt die deutsche Finanzverwaltung häufig die Auffassung, bei einem Cash Pool Master handele es sich um eine Dienstleistung, die aufgrund ihres einfachen Funktionsprofils lediglich nach der Kostenaufschlagsmethode zu vergüten ist. ${ }^{67}$ Sämtliche Synergiegewinne des Pools, die sich aus der Bündelung der Bedürfnisse nach Finanzierungsleistungen der Cash Pool Mitglieder ergeben, sind demzufolge auf die Mitglieder aufzuteilen (siehe hierzu auch Ausführungen zur Verteilung von Synergiegewinne in Kap. 5.4.1.2). Diese pauschale Sichtweise, dass alle Cash Pool Master lediglich Routinefunktionen ausüben, die anhand der Kostenaufschlagsmethode zu vergüten sind, ist in der Praxis und Literatur jedoch stark umstritten. ${ }^{68}$ Schließlich hängt die Auswahl der angemessenen Verrechnungspreismethode jeweils von den tatsächlich ausgeübten Funktionen und getragenen Risiken der involvierten Parteien sowie der spezifischen Ausgestaltung des Cash Pools ab. Daher ist eine umfangreiche Funktions- und Risikoanalyse sowie der Umstände des Einzelfalls un-

${ }^{67}$ Vgl. Bubeck, D. \& Schreiber, R., Fremdvergleich beim internationalen Cash Pool, Der Betrieb, Nr. 18, 2.5.2014.

${ }^{68}$ Vgl. Hülshorst, J., Kuzmina, S. \& Wehke, S., Fremdübliche Methodenwahl beim Cash Pooling, Der Betrieb vom 22.08.2014, Heft 34, Seite 1887-1892, DB0664930. 
erlässlich, um eine Verrechnungspreismethode auszuwählen, die zu einer fremdüblichen Aufteilung der Gewinne im Cash Pool führt.

Neben den Konditionen des Cash Pools und der damit verbundenen Verteilung der Gewinne des Pools ist auch die Klassifizierung von einzelnen Transaktionen entweder als Teil des Cash Pools oder als Darlehenstransaktion von Relevanz. So sollten fremdüblichen Kriterien (bspw. Laufzeit und Höhe) festgelegt werden, die bestimmen ob eine Transaktion unter Cash Pool Transaktion oder eine anderweitige Darlehenstransaktion fällt. Bspw. könnten längerfristige, hohe Einlagen oder Ausleihungen eines Cash Pool Mitglieds unter marktüblichen Kriterien eher unter ein Darlehen als ein Guthaben oder Überziehungstransaktion fallen, das wahrscheinlich höher zu verzinsen ist.

\subsubsection{Bürgschaften/Garantien}

Die Vergabe von Garantien an verbundene Unternehmen unterliegt, wie alle schuldrechtlichen Transaktionen, ebenfalls dem Fremdvergleichsgrundsatz. Provisionen für Garantien oder Bürgschaften sind dem Grunde nach zu verrechnen, wenn sie auch zwischen fremden Dritten vereinbart worden wären und der Grund für die Gewährung der Garantie nicht im Gesellschaftsverhältnis begründet liegt. Dies ist bspw. der Fall, wenn die Garantie dem Schuldner einen konkreten Nutzen bringt, wie z. B. die Reduzierung von Finanzierungskosten oder den Zugang zu einem bestimmten Kapitalmarkt. ${ }^{69}$ Nach Sichtweise der OECD liegt bei der Gewährung einer Garantie an ein verbundenes Unternehmen eine konzerninterne Dienstleistung vor, die zu verrechnen ist, sofern dem Begünstigten dadurch ein Nutzen entsteht. ${ }^{70}$ Dies erfordert eine entsprechende bewusste und absichtliche Handlung, in der Regel die Ausstellung einer schriftlichen expliziten Garantie. Eine Provision ist erst dann für eine Garantie zu verlangen, sofern diese die Refinanzierungskosten des Garantieempfängers weitergehend mindert, als durch den bereits bestehenden Rückhalt im Konzern.

Als prominentes Beispiel ist hier der Fall von General Electric Capital Canada Inc. zu nennen. ${ }^{71}$ General Electric Capital Canada Inc. bezahlte in den Jahren von 1996 bis 2000 eine Garantiegebühr von $1 \%$ auf sämtliche Ausleihbeträge an ihre Muttergesellschaft GE Capital Corporation Inc., USA. Es handelte sich dabei in diesem Zeitraum insgesamt um 136 Mio. kanadische Dollar. Die kanadische Finanzverwaltung hat die komplette Garantiegebühr als nicht steuerlich abzugsfähig anerkannt, mit der Begründung, es handele sich aufgrund der Mutter-Tochter Beziehung der beiden Unternehmen um Rückhalt im Konzern (,implicit support“). Schließlich hat der Tax Court of Canada nach einem langwierigem Prozess im Sinne von General Electric Capital Canada Inc. entschieden und die gezahlte Garantiegebühr als steuerlich abzugsfähige Ausgabe akzeptiert. Die Begründung lautete, dass Konzernrückhalt zwar schon von wirtschaftlicher Bedeutung ist und beachtet

\footnotetext{
${ }^{69}$ Vgl. Tz. 4.4 VWG 1983.

${ }^{70}$ Vgl. Tz. 7.13 VWG 1983, Aligning Transfer Pricing Outcomes with Value Creation, Actions 8-10.

${ }^{71}$ GE Capital Canada Inc. v. Her Majesty the Queen, 2009 DTC 563.
} 
werden müsse, andererseits ist dies nicht mit einer expliziten Garantie oder Bürgschaft $\mathrm{zu}$ vergleichen, wie diese im Fall von General Electric Capital Canada Inc. durch die Muttergesellschaft ausgesprochen wurde. Bei der Feststellung, ob die 1\% Garantiegebühr fremdüblich ist, wurde durch die Richter betrachtet, inwieweit sich die Kreditwürdigkeit der kanadischen Tochtergesellschaft durch die explizite Bürgschaft verbessert hatte und inwieweit dabei Zinsvorteile bei der Aufnahme von Fremdkapital durch die kanadische Gesellschaft erzielt werden konnten. Hierzu wurde die Kreditwürdigkeit der General Electric Capital Canada Inc. als eigenständiges Unternehmen bestimmt, wobei ein Rating von $\mathrm{B}+$ bis $\mathrm{BB}$ - erzielt wurde. Das entsprechende Rating wurde um drei Kategorien (Notches) nach oben gestuft (d. h. BB + bis BBB -), um dem Umstand des Konzernrückhalts Rechnung zu tragen bzw. der Tatsache, dass die Kreditwürdigkeit der General Electric Capital Canada Inc. auch in Teilen von der Entscheidung über die Eigenkapitalquote bzw. Ausschüttung von Gewinnen durch die Muttergesellschaft abhängig ist. Schließlich wurden die Zinskonditionen der General Electric Capital Canada Inc. unter diesem Rating mit dem tatsächlichen Rating des Konzerns (höchste Kategorie oder AAA) verglichen. Dabei stellte sich heraus, dass der erzielte Zinsvorteil der General Electric Capital Canada Inc. höher war als die $1 \%$ Garantiegebühr, die von der General Electric Capital Canada Inc. an die Muttergesellschaft gezahlt wurde. Somit wurde der Klage der General Electric Capital Canada Inc. gegen die ursprüngliche vollständige Nichtanerkennung durch die Finanzverwaltung stattgegeben.

Dieses Beispiel zeigt, welche Faktoren bei der ökonomischen Analyse von Garantiegebühren oder Bürgschaften von Relevanz sind. Zum einen ist eine explizite vertragliche Garantie oder Bürgschaft von einem Konzernrückhalt zu unterscheiden, da der Garantiegeber oder Bürge schriftlich fixierte, explizite Risiken eingeht, die unter Umständen zusätzlich die eigene Kreditwürdigkeit beschränken. Zum anderen entstehen beim Empfänger der Garantie Vorteile in Form von besseren Konditionen in Finanzierungstransaktionen, für die ein fremder Dritter bereit wäre, eine Gebühr zu entrichten und üblicherweise zwischen unverbundenen Dritten auch eine entsprechende Gebühr entrichtet wird. Insofern ist grundsätzlich davon auszugehen, dass eine Gebühr für die Ausgabe einer expliziten Bürgschaft oder Garantie unter dem Fremdvergleichsgrundsatz gerechtfertigt ist.

Entsprechend ist die übliche Herangehensweise bei der Bestimmung einer markt- bzw. fremdüblichen Garantiegebühr, die Vorteile des Garantienehmers bzw. die resultierenden Nachteile des Garantiegebers zu betrachten (sog. „Yield Approach“). Die Vorteile des Garantienehmers lassen sich in der Regel aus einem Vergleich der Finanzierungskonditionen des Garantieempfängers ohne Garantie im Vergleich zu den Konditionen mit Garantie bestimmen. Die Finanzierungskonditionen ohne Garantie sollten sich dabei an der individuellen Kreditwürdigkeit des Garantienehmers („stand-alone Rating“) orientieren, wobei ein möglicher Einfluss der Konzernzugehörigkeit auf die Kreditwürdigkeit berücksichtigt werden solle. ${ }^{72}$ Bei der Ermittlung der Finanzierungskonditionen

\footnotetext{
${ }^{72}$ Vgl. GE Capital Fall bzw. Beispiele 1 und 2 in Tz. 1.164 - 1.167 in OECD (2015), Aligning Transfer Pricing Outcomes with Value Creation, Actions 8-10.
} 
mit Garantie ist davon auszugehen, dass sich bei Ausstellung einer expliziten Garantie durch ein anderes Konzernunternehmen (in der Regel die Konzernmutter) das Rating des Garantieempfängers auf das Rating des Garantieausgebers verbessert hat. Die resultierenden Zinsvorteile wären dann der maximale Betrag, den der Empfänger bereit wäre, für die Garantie zu bezahlen. Aus Sicht des Garantiegebers wäre ein möglicher Ansatz zur Bestimmung einer fremdüblichen Garantiegebühr einerseits zu analysieren, welche impliziten Kosten dem Garantiegeber (d. h. Kapitalkosten) durch Ausgabe der Garantie entstehen (sog. „expected-loss-Methode“). Die Kosten des Garantiegebers können sich im Prinzip aus dem nominalen Garantiebetrag multipliziert mit der Ausfallwahrscheinlichkeit des Garantienehmers und den Kapitalkosten berechnen.

Schließlich besteht zudem die Möglichkeit, eine fremdübliche Garantiegebühr anhand der Preisvergleichsmethode zu bestimmen. Dies setzt voraus, dass entsprechende zuverlässige Vergleichsdaten vorliegen. Vorsicht sei jedoch bei der Verwendung von Garantiegebühren, die Banken für die Ausgabe von Garantiegebühren berechnen, geboten. Banken verfolgen in der Regel andere Geschäftsstrategien und Kapitalkosten als die betrachteten konzerninternen Garantiegeber, so dass eine Vergleichbarkeit der Transaktionen nicht automatisch gegeben ist. Aufgrund der beschriebenen Schwierigkeiten hinsichtlich der Vergleichbarkeit werden in der Praxis zumeist der Yield Approach bzw. die ,expected-loss Methode" angewendet.

\section{Literatur}

Announcement and report concerning advance pricing agreements vom 30. März, 2014. Internal Revenue Services (IRS). http://www.irs.gov/pub/irs-drop/a-15-11.pdf. Zugegriffen: 22.11.2015

BFH, Urteil vom 17.2.1993. Verdeckte Gewinnausschüttung bei Verzicht auf branchenüblichen Werbekostenausgleich. BStB1 II 1993, S. 457 BFH, Urteil vom 28.2.1990. Für Zwecke des summarischen Aussetzungsverfahrens ist es nicht zu beanstanden, wenn die Höhe einer verdeckten Gewinnausschüttung, die in einer zinslosen Darlehensgewährung besteht, nach den Habenzinsen für Drei-Monats-Festgelder des Zeitraums ermittelt wird, für den das Darlehen zinslos gewährt wird. BStB1. 1990 II, S. 649

BMF, Schreiben vom 30.12.1999, - IV B 4- S 1341-14/99. Grundsätze für die Prüfung der Einkunftsabgrenzung durch Umlageverträge zwischen international verbundenen Unternehmen. BStB1 1999 I S. 112

BMF, Schreiben vom 12.4.2005. Grundsätze für die Prüfung der Einkunftsabgrenzung zwischen nahestehenden Personen mit grenzüberschreitenden Geschäftsbeziehungen in Bezug auf Ermittlungs- und Mitwirkungspflichten, Berichtigungen sowie auf Verständigungs- und EU Schiedsverfahren (Verwaltungsgrundsätze-Verfahren). IV B4- S 1341-1/05. BStB1 2005 I, S. 570

BMF, Schreiben vom 17.2.1983. Grundsätze für die Prüfung der Einkunftsabgrenzung bei international verbundenen Unternehmen (Verwaltungsgrundsätze), IV C 5- S 1341-4/83. BStB1 1983 I S. 218 (Geändert durch BMF v. 30. 12. 1999 BStBl. I S. 1122)

Bubeck, D. \& Schreiber, R., Fremdvergleich beim internationalen Cash Pool, Der Betrieb, Nr. 18, 2.5.2014

Ebering, A., Wann sind Preisanpassungen bei Funktionsverlagerungen i. S. v. § 1 Abs. 3 Satz 9 AStG fremdüblich?, IStR 11/2011, S. 418 ff. 
GE Capital Canada Inc. v. Her Majesty the Queen, 2009 DTC 563

Hülshorst, J., Kuzmina, S. \& Wehke, S., Fremdübliche Methodenwahl beim Cash Pooling, Der Betrieb vom 22.08.2014, Heft 34, Seite 1887-1892, DB0664930

IRS Regulations zu "Services Cost Method", § 1.482-9(b)(2). 2007. Internal Revenue Bulletin: 2007-3.0ECD (Hrsg.)

OECD (Hrsg.). 22. Juli, 2010. Transfer Pricing Guidelines for Multinational Enterprises and Tax Administrations. OECD Publishing ("OECD-RL")

OECD (2015), Aligning Transfer Pricing Outcomes with Value Creation, Actions 8-10 - 2015 Final Reports, OECD/G20 Base Erosion and Profit Shifting Project, OECD Publishing, Paris ("OECD (2015), Aligning Transfer Pricing Outcomes with Value Creation, Actions 8-10") http://dx.doi. org/10.1787/9789264241244-en

OECD (2015), Transfer Pricing Documentation and Country-by-Country Reporting, Action 13 2015 Final Report, OECD/G20 Base Erosion and Profit Shifting Project, OECD Publishing, Paris ("OECD (2015), Transfer Pricing Documentation and Country-by-Country Reporting, Action 13”) http://dx.doi.org/10.1787/9789264241480-en

Daniel Schwerdt arbeitet als Senior Manager im Bereich Verrechnungspreise bei PwC am Standort Frankfurt am Main. Herr Schwerdt ist Diplom Volkswirt und hat Volkswirtschaftslehre an der Universität Konstanz und der Carleton University, Ottawa (Kanada) studiert. Zudem ist er Chartered Financial Analyst (CFA) und Mitglied der CFA Society. Herr Schwerdt berät seit über 10 Jahren multinationale Unternehmen bei der Planung, Verteidigung und Dokumentation von Verrechnungspreissystemen.

Open Access Dieses Kapitel wird unter der Creative Commons Namensnennung-Nicht kommerziell 2.5 International Lizenz (http://creativecommons.org/licenses/by-nc/2.5/deed.de) veröffentlicht, welche für nicht kommerzielle Zwecke die Nutzung, Verbreitung und Wiedergabe in jeglichem Medium und Format erlaubt, sofern Sie den/die ursprünglichen Autor(en), den Titel des Werks und die Quelle ordnungsgemäß nennen, einen Link zur Creative Commons Lizenz beifügen und im Falle einer Abwandlung durch einen entsprechenden Hinweis deutlich erkennbar machen, dass Änderungen vorgenommen wurden.

Die in diesem Kapitel enthaltenen Bilder und sonstiges Drittmaterial unterliegen ebenfalls der genannten Creative Commons Lizenz, sofern sich aus der Abbildungslegende nichts anderes ergibt. Sofern das betreffende Material nicht unter der genannten Creative Commons Lizenz steht und die betreffende Handlung nicht nach gesetzlichen Vorschriften erlaubt ist, ist auch für die oben aufgeführten nichtkommerziellen Weiterverwendungen des Materials die Einwilligung des jeweiligen Rechteinhabers einzuholen. 